\title{
Computational and Experimental Analysis on the Conformational Preferences of Anticancer Saponin OSW-1
}

\author{
Keisuke Fukaya, ${ }^{1}$ Daisuke Urabe,${ }^{*, 1}$ Masato Hiraizumi, ${ }^{2}$ Keiichi Noguchi, ${ }^{2}$ Takashi \\ Matsumoto, ${ }^{2}$ Kaori Sakurai*,2
${ }^{1}$ Biotechnology Research Center and Department of Biotechnology, Toyama Prefectural University, 5180 Kurokawa, Imizu, Toyama 939-0398, Japan
${ }^{2}$ Department of Biotechnology and Life Science, Tokyo University of Agriculture and Technology, Koganei, Tokyo 184-8588, Japan

Supporting Information

41 pages

Contents:

- Figure S1. X-ray crystallographic structure of OSW-1 (1) with thermal ellipsoids at the $50 \%$ probability level.

- Table S1. Crystallographic data of $\mathbf{1}$

- Table S2. Cartesian coordinates of the optimized structures of 1-Ba-l and 1-C

- Figure S2. Calculated ECD spectra of conformers in 1-Ba-1, 1-C, and the non-disordered monomer in crystal structure

- Table S3. Comparison of experimental and calculated NMR chemical shifts, the DP4+ analysis, and CMAE

- Table S4. Calculated NMR chemical shifts of each conformer in 1-B 


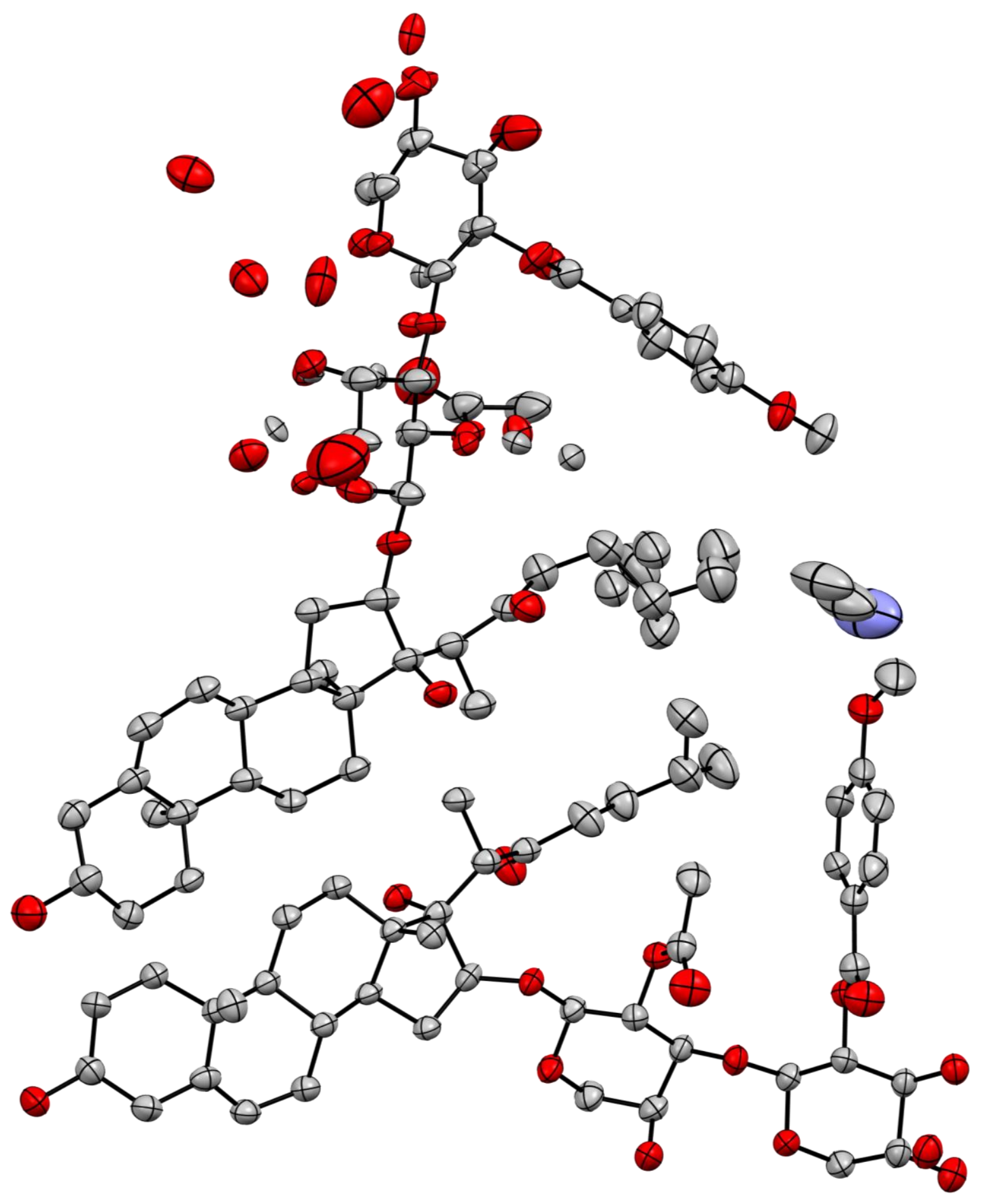

Figure S1. X-ray crystallographic structure of OSW-1 (1) with thermal ellipsoids at the $50 \%$ probability level. 
Table S1. Crystallographic data of 1

\begin{tabular}{|c|c|}
\hline Compound & OSW-1 \\
\hline CCDC number & 1907483 \\
\hline Molecular formula & $\begin{array}{l}2\left(\mathrm{C}_{47} \mathrm{H}_{68} \mathrm{O}_{15}\right), \\
\mathrm{C}_{2} \mathrm{H}_{3} \mathrm{~N}, 5.579\left(\mathrm{H}_{2} \mathrm{O}\right)\end{array}$ \\
\hline Formula weight & 1887.60 \\
\hline Temperature $(\mathrm{K})$ & 93 \\
\hline Wavelength $(\AA)$ & 1.54187 \\
\hline Crystal color, habit & colorless, needle \\
\hline Crystal size $\left(\mathrm{mm}^{3}\right)$ & $0.35 \times 0.03 \times 0.03$ \\
\hline Crystal system & orthorhombic \\
\hline Space group & $P 2{ }_{1} 2_{1} 2_{1}(\# 19)$ \\
\hline Unit cell dimensions & \\
\hline$a(\AA)$ & $6.5917(10)$ \\
\hline$b(\AA)$ & $29.503(5)$ \\
\hline$c(\AA)$ & $50.546(8)$ \\
\hline$\alpha\left(^{\circ}\right)$ & 90.00 \\
\hline$\beta\left(^{\circ}\right)$ & 90.00 \\
\hline$\gamma\left({ }^{\circ}\right)$ & 90.00 \\
\hline Volume $\left(\AA^{3}\right)$ & $9830(3)$ \\
\hline$Z$ & 4 \\
\hline $\begin{array}{l}\text { Density (calculated) } \\
\left(\mathrm{g} / \mathrm{cm}^{3}\right)\end{array}$ & 1.275 \\
\hline$\mu(\mathrm{CuK} \alpha)\left(\mathrm{cm}^{-1}\right)$ & 0.802 \\
\hline $\mathrm{F}(000)$ & 4071 \\
\hline Index ranges & $\begin{array}{l}-7<=\mathrm{h}<=6 \\
-35<=\mathrm{k}<=34 \\
-60<=1<=51\end{array}$ \\
\hline Reflections collected & 54337 \\
\hline Independent reflections & 17652 \\
\hline$R($ int $)$ & 0.0730 \\
\hline Completeness to theta & $67.687^{\circ}, 99.5 \%$ \\
\hline Refinement method & $\begin{array}{l}\text { Full-matrix } \\
\text { least-squares on } \mathrm{F}^{2}\end{array}$ \\
\hline $\begin{array}{l}\text { No. Observations } \\
\text { (All reflections) }\end{array}$ & 17652 \\
\hline No. Variables & 1457 \\
\hline Reflection/Parameter Ratio & 6.98 \\
\hline Goodness-of-fit on $\mathrm{F}^{2}$ & 1.068 \\
\hline Residuals: $R 1(I>2.00 \sigma(I))$ & 0.0720 \\
\hline $\begin{array}{l}\text { Residuals: } R \\
\text { (All reflections) }\end{array}$ & 0.0887 \\
\hline $\begin{array}{l}\text { Residuals: wR2 } \\
\text { (All reflections) }\end{array}$ & 0.2219 \\
\hline $\begin{array}{l}\text { Max. and min. peak } \\
\text { in Final Diff. Map }\left(\mathrm{e}^{-} / \AA^{3}\right)\end{array}$ & $0.594,-0.652$ \\
\hline
\end{tabular}


Table S2. Cartesian coordinates of the optimized structures of 1-Ba-l and 1-C

Structure, energy and Cartesian coordinate of 1-Ba:

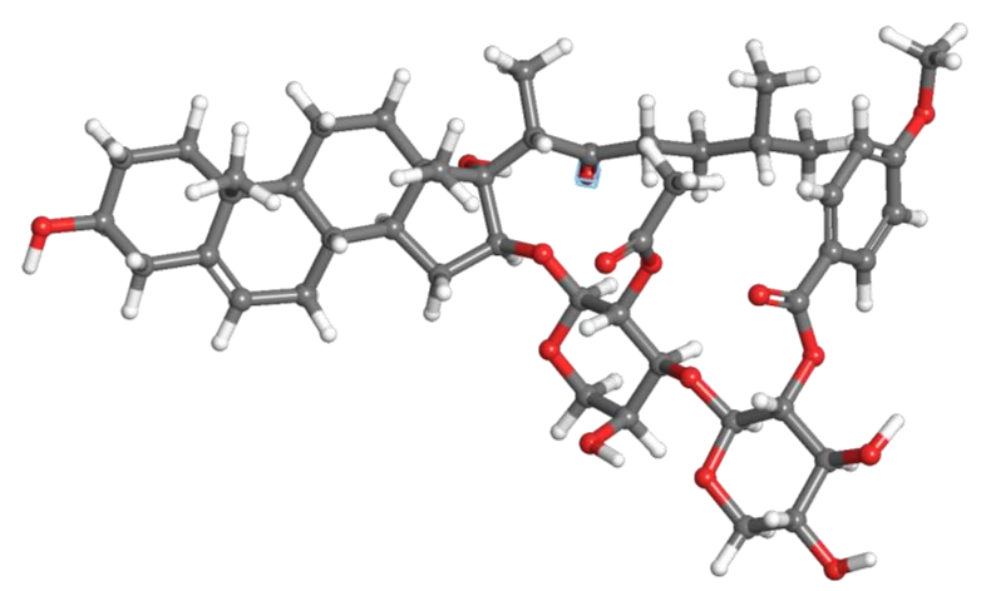

$1-\mathrm{Ba}(\Delta G=0.0 \mathrm{kcal} / \mathrm{mol}$, Boltzmann pop. $(298 \mathrm{~K})=32.7 \%)$

M06-2X/6-311+G(d,p)-PCM(MeOH)//M06-2X/6-31G(d)-PCM(MeOH):

\begin{aligned} Gibbs Free Energy (a.u.) & $=-2958.9946920 \\$\hline M06-2X/6-311+G(d,p)-PCM(MeOH): $\quad$ Electronic energy (a.u.) & $=-2960.0352050 \\$\hline M06-2X/6-31G(d)-PCM(MeOH): Zero-point correction (a.u.) & $=1.134864 \\$ Thermal correction to Energy (a.u.) & $=1.195079 \\$ Thermal correction to Enthalpy (a.u.) & $=1.196023 \\$ Thermal correction to Gibbs Free Energy (a.u.) & $=1.040513\end{aligned}$

$\begin{array}{lrrrrrrr}\mathrm{O} & -0.89441 & -0.08198 & 0.62541 & \mathrm{H} & 7.76532 & 5.87343 & -0.39017 \\ \mathrm{O} & 3.03994 & 2.37364 & -0.04240 & \mathrm{H} & -12.07597 & 2.63082 & -0.64806 \\ \mathrm{O} & 5.70145 & 1.55212 & -0.58542 & \mathrm{H} & 2.50594 & -1.15205 & -3.27363 \\ \mathrm{O} & 1.70403 & 0.00118 & -0.37833 & \mathrm{H} & 1.31969 & -2.08403 & -2.33676 \\ \mathrm{O} & -3.22724 & -2.34719 & 2.16523 & \mathrm{H} & 2.91007 & -1.72726 & -1.62536 \\ \mathrm{O} & -0.47309 & 1.73323 & 1.95199 & \mathrm{H} & 2.78907 & 1.22182 & 1.65439 \\ \mathrm{O} & 1.00615 & 3.99092 & 0.98951 & \mathrm{H} & -3.30802 & 1.32881 & 2.02106 \\ \mathrm{O} & 3.76595 & 4.47271 & 0.38761 & \mathrm{H} & -2.87226 & 1.30002 & 0.31151 \\ \mathrm{O} & 7.41348 & 3.77889 & -1.37563 & \mathrm{H} & -6.14492 & 1.40189 & 2.01766 \\ \mathrm{O} & 7.00943 & 6.07650 & 0.18501 & \mathrm{H} & -5.34537 & 2.53546 & 0.94688 \\ \mathrm{O} & -11.90995 & 1.78199 & -1.08477 & \mathrm{H} & -4.81457 & -0.54726 & 1.76576 \\ \mathrm{O} & -0.53607 & -2.73015 & 2.56357 & \mathrm{H} & -7.09745 & -0.71415 & 0.87983 \\ \mathrm{O} & 0.96368 & 0.76868 & -2.36956 & \mathrm{H} & 4.84916 & 3.09441 & -1.68342 \\ \mathrm{O} & 4.43382 & 0.89878 & -2.34167 & \mathrm{H} & -1.20782 & -2.27544 & -0.52818 \\ \mathrm{O} & 7.05124 & -4.55017 & -0.30363 & \mathrm{H} & -5.29136 & -2.71611 & 0.96422 \\ \mathrm{C} & 2.12081 & -1.33957 & -2.27110 & \mathrm{H} & -4.70173 & -3.29361 & -0.59430 \\ \mathrm{C} & 2.20764 & 1.89048 & 1.00114 & \mathrm{H} & -7.02617 & -2.50310 & -0.77961 \\ \mathrm{C} & -3.17104 & 0.67143 & 1.15851 & \mathrm{H} & -5.94141 & -1.59019 & -1.80718 \\ \mathrm{C} & -2.66835 & -1.76757 & 0.99300 & \mathrm{H} & 0.55363 & -0.04714 & 2.08130 \\ \mathrm{C} & -6.07525 & 1.71576 & 0.96356 & \mathrm{H} & -5.22606 & 0.95056 & -0.84429\end{array}$




\begin{tabular}{|c|c|c|c|c|c|c|c|}
\hline $\mathrm{C}$ & -4.41174 & -0.15529 & 0.82000 & $\mathrm{H}$ & 0.59835 & 1.75004 & -0.40937 \\
\hline $\mathrm{C}$ & -0.40487 & -2.81690 & 1.35061 & $\mathrm{H}$ & 2.35391 & 3.46824 & 2.47963 \\
\hline $\mathrm{C}$ & -6.71276 & -0.45987 & -0.12313 & $\mathrm{H}$ & -7.72597 & 3.19226 & 0.87144 \\
\hline $\mathrm{C}$ & -3.85821 & -1.38558 & 0.06606 & $\mathrm{H}$ & -4.05654 & -0.49373 & -1.92750 \\
\hline $\mathrm{C}$ & 5.24576 & 2.89967 & -0.68315 & $\mathrm{H}$ & -3.04061 & -1.93155 & -1.87378 \\
\hline $\mathrm{C}$ & -1.59250 & -2.70218 & 0.40303 & $\mathrm{H}$ & -2.42357 & -0.38784 & -1.26647 \\
\hline $\mathrm{C}$ & -5.00513 & -2.39644 & -0.04391 & $\mathrm{H}$ & -1.77637 & -0.42800 & 2.45490 \\
\hline $\mathrm{C}$ & -6.21180 & -1.76987 & -0.76085 & $\mathrm{H}$ & 4.49998 & 2.79306 & 1.34575 \\
\hline $\mathrm{C}$ & 0.08923 & 0.60709 & 1.31584 & $\mathrm{H}$ & 6.85283 & 3.50561 & 0.60168 \\
\hline $\mathrm{C}$ & -5.56851 & 0.54587 & 0.11930 & $\mathrm{H}$ & -10.40172 & 0.07891 & -2.31182 \\
\hline $\mathrm{C}$ & 1.54189 & -0.06724 & -1.72124 & $\mathrm{H}$ & -11.29572 & -0.75909 & -1.04285 \\
\hline $\mathrm{C}$ & -8.21493 & 1.56663 & -0.34553 & $\mathrm{H}$ & -6.59642 & 0.74309 & -2.56611 \\
\hline $\mathrm{C}$ & 1.11477 & 1.10315 & 0.30228 & $\mathrm{H}$ & -8.31300 & 0.92936 & -2.92086 \\
\hline $\mathrm{C}$ & 1.58358 & 3.02173 & 1.83264 & $\mathrm{H}$ & -7.60303 & -0.68186 & -2.89319 \\
\hline $\mathrm{C}$ & -7.40923 & 2.21862 & 0.49724 & $\mathrm{H}$ & -8.99605 & -1.67726 & -1.20015 \\
\hline $\mathrm{C}$ & -3.31386 & -1.02222 & -1.32528 & $\mathrm{H}$ & -9.27256 & -0.91616 & 0.36507 \\
\hline $\mathrm{C}$ & -2.06479 & -0.37594 & 1.39986 & $\mathrm{H}$ & 5.21196 & 5.04505 & 1.77137 \\
\hline $\mathrm{C}$ & -7.90025 & 0.17692 & -0.90047 & $\mathrm{H}$ & 4.42267 & 6.33954 & 0.83439 \\
\hline $\mathrm{C}$ & 4.14201 & 3.10705 & 0.34900 & $\mathrm{H}$ & 0.93076 & 1.78382 & 3.47969 \\
\hline $\mathrm{C}$ & 6.42563 & 3.80605 & -0.36823 & $\mathrm{H}$ & -0.04738 & 3.25216 & 3.21624 \\
\hline $\mathrm{C}$ & -10.45125 & -0.08613 & -1.22741 & $\mathrm{H}$ & 5.04660 & -1.47795 & -3.02736 \\
\hline $\mathrm{C}$ & -7.58437 & 0.29815 & -2.40550 & $\mathrm{H}$ & -10.81495 & 1.08043 & 0.53403 \\
\hline $\mathrm{C}$ & -9.15559 & -0.71145 & -0.70847 & $\mathrm{H}$ & -9.50666 & 2.41149 & -1.86298 \\
\hline $\mathrm{C}$ & 4.83145 & 5.33007 & 0.77961 & $\mathrm{H}$ & -9.68105 & 3.13543 & -0.26298 \\
\hline $\mathrm{C}$ & 0.48850 & 2.44291 & 2.71828 & $\mathrm{H}$ & 5.56876 & 5.57120 & -1.22559 \\
\hline $\mathrm{C}$ & 5.52636 & -1.72681 & -2.08597 & $\mathrm{H}$ & -2.76206 & -4.12335 & -0.75782 \\
\hline $\mathrm{C}$ & -10.70749 & 1.24856 & -0.55042 & $\mathrm{H}$ & -2.69320 & -4.48233 & 0.97992 \\
\hline $\mathrm{C}$ & 5.68974 & -0.72849 & -1.12525 & $\mathrm{H}$ & -1.29747 & -4.81894 & -0.06572 \\
\hline $\mathrm{C}$ & 5.18626 & 0.63094 & -1.43300 & $\mathrm{H}$ & 5.83809 & -3.77992 & -2.61834 \\
\hline $\mathrm{C}$ & -9.52279 & 2.18514 & -0.78732 & $\mathrm{H}$ & 0.81839 & -3.81167 & -0.07262 \\
\hline $\mathrm{C}$ & 5.95521 & 5.24892 & -0.24641 & $\mathrm{H}$ & 1.20743 & -2.12762 & 0.21366 \\
\hline $\mathrm{C}$ & -2.11813 & -4.12008 & 0.12419 & $\mathrm{H}$ & 6.45716 & -0.26543 & 0.83750 \\
\hline $\mathrm{C}$ & 5.97349 & -3.02241 & -1.85592 & $\mathrm{H}$ & 7.25419 & -2.58386 & 1.26388 \\
\hline $\mathrm{C}$ & 0.94399 & -3.06548 & 0.72228 & $\mathrm{H}$ & 1.71737 & -4.37991 & 2.23653 \\
\hline $\mathrm{C}$ & 6.32090 & -1.03680 & 0.08773 & $\mathrm{H}$ & 2.09980 & -2.68442 & 2.48708 \\
\hline $\mathrm{C}$ & 6.59315 & -3.32301 & -0.63821 & $\mathrm{H}$ & 3.74891 & -2.75094 & 0.63137 \\
\hline $\mathrm{C}$ & 6.76499 & -2.32321 & 0.33122 & $\mathrm{H}$ & 2.90238 & -5.68646 & 0.39714 \\
\hline $\mathrm{C}$ & 2.02605 & -3.46184 & 1.71687 & $\mathrm{H}$ & 4.39259 & -5.02993 & -0.29858 \\
\hline $\mathrm{C}$ & 3.40767 & -3.69237 & 1.09273 & $\mathrm{H}$ & 2.82414 & -4.45670 & -0.88086 \\
\hline $\mathrm{C}$ & 3.37430 & -4.77363 & 0.01123 & $\mathrm{H}$ & 5.39243 & -4.28162 & 1.76514 \\
\hline $\mathrm{C}$ & 4.40282 & -4.07998 & 2.18723 & $\mathrm{H}$ & 4.06528 & -4.99024 & 2.69866 \\
\hline $\mathrm{C}$ & 7.04498 & -5.55823 & -1.30505 & $\mathrm{H}$ & 4.50139 & -3.28850 & 2.93802 \\
\hline $\mathrm{H}$ & -2.46475 & -2.54145 & 2.74094 & $\mathrm{H}$ & 7.51305 & -6.43116 & -0.85262 \\
\hline $\mathrm{H}$ & 1.74641 & 4.39721 & 0.50458 & $\mathrm{H}$ & 7.62121 & -5.23994 & -2.1798 \\
\hline $\mathrm{H}$ & 7.86230 & 2.92072 & -1.33763 & $\mathrm{H}$ & 6.02377 & -5.80907 & -1.6101 \\
\hline
\end{tabular}


Structure, energy and Cartesian coordinate of $\mathbf{1 - B b}$ :

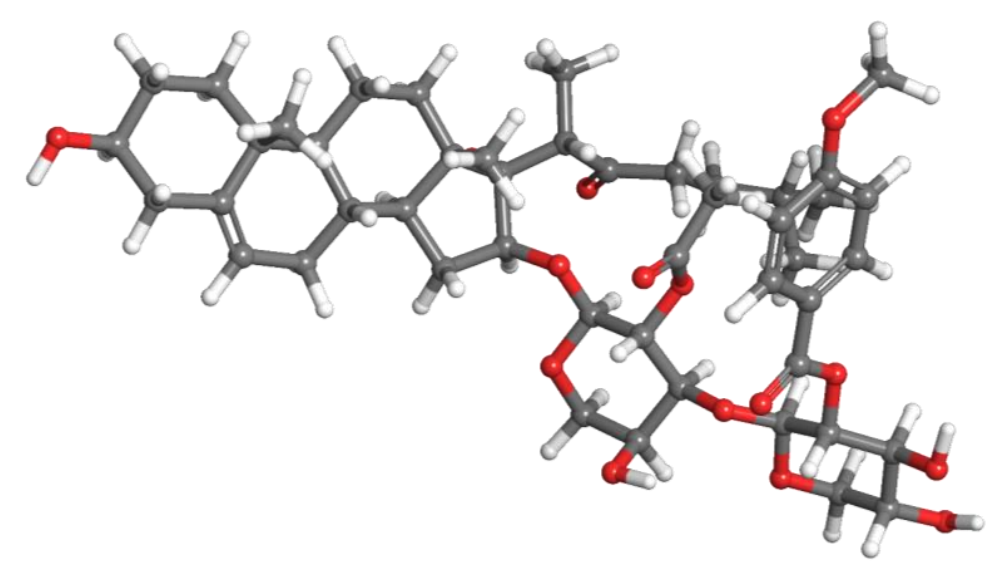

1-Bb $(\Delta G=0.6 \mathrm{kcal} / \mathrm{mol}$, Boltzmann pop. $(298 \mathrm{~K})=12.4 \%)$

M06-2X/6-311+G(d,p)-PCM(MeOH)//M06-2X/6-31G(d)-PCM(MeOH):

Gibbs Free Energy (a.u.) $=-2958.9937771$

M06-2X/6-311+G(d,p)-PCM(MeOH): $\quad$ Electronic energy (a.u. $)=-2960.0316231$

M06-2X/6-31G(d)-PCM(MeOH): Zero-point correction (a.u.) $=1.134424$

Thermal correction to Energy (a.u.) $=1.195055$

Thermal correction to Enthalpy (a.u.) $=1.195999$

Thermal correction to Gibbs Free Energy (a.u.) = 1.037846

$\begin{array}{lrrr}\mathrm{O} & 0.72418 & -1.04965 & 0.33779 \\ \mathrm{O} & -3.59505 & -1.37806 & -1.54056 \\ \mathrm{O} & -5.44033 & 0.76698 & -1.33025 \\ \mathrm{O} & -1.84922 & -0.05826 & 0.21267 \\ \mathrm{O} & 3.23350 & -1.71492 & 2.85578 \\ \mathrm{O} & 0.09960 & -2.95478 & -0.77086 \\ \mathrm{O} & -1.85751 & -2.96756 & -2.89438 \\ \mathrm{O} & -5.30905 & -2.86686 & -1.53008 \\ \mathrm{O} & -8.11388 & 0.06214 & -2.20354 \\ \mathrm{O} & -8.91501 & -2.50603 & -1.38618 \\ \mathrm{O} & 11.52486 & 0.55058 & -2.46151 \\ \mathrm{O} & 0.63943 & -2.14668 & 3.58209 \\ \mathrm{O} & -0.94878 & 1.42965 & -1.22773 \\ \mathrm{O} & -4.10083 & 1.16169 & -3.10616 \\ \mathrm{O} & -3.36811 & 6.51230 & 0.21814 \\ \mathrm{C} & -1.79564 & 2.19350 & 0.89756 \\ \mathrm{C} & -2.62111 & -2.08968 & -0.79099 \\ \mathrm{C} & 2.99104 & -1.71866 & -0.28922 \\ \mathrm{C} & 2.55513 & -0.81219 & 1.99237 \\ \mathrm{C} & 5.83718 & -1.67829 & -1.49892 \\ \mathrm{C} & 4.22492 & -1.04708 & 0.31726 \\ \mathrm{C} & 0.39143 & -0.98687 & 3.28314\end{array}$

$\begin{array}{rrrr}\mathrm{H} & -9.47864 & -1.78420 & -1.70938 \\ \mathrm{H} & 11.69970 & -0.12405 & -3.13462 \\ \mathrm{H} & -2.68994 & 1.91242 & 1.45670 \\ \mathrm{H} & -1.91807 & 3.17842 & 0.44560 \\ \mathrm{H} & -0.94885 & 2.22728 & 1.59267 \\ \mathrm{H} & -3.03282 & -2.37696 & 0.18717 \\ \mathrm{H} & 3.17642 & -2.74272 & -0.62441 \\ \mathrm{H} & 2.61714 & -1.15210 & -1.14980 \\ \mathrm{H} & 5.98775 & -2.57230 & -0.87226 \\ \mathrm{H} & 5.08742 & -1.96564 & -2.24753 \\ \mathrm{H} & 4.71291 & -1.78638 & 0.96897 \\ \mathrm{H} & 6.91003 & -0.76310 & 0.73441 \\ \mathrm{H} & -5.61585 & -0.56883 & -2.90803 \\ \mathrm{H} & 0.98112 & 0.67236 & 2.10676 \\ \mathrm{H} & 5.18962 & -0.29692 & 2.74258 \\ \mathrm{H} & 4.49935 & 1.32493 & 2.81536 \\ \mathrm{H} & 6.77503 & 1.39175 & 1.88408 \\ \mathrm{H} & 5.58829 & 1.98897 & 0.74425 \\ \mathrm{H} & -0.67129 & -2.38044 & 1.04599 \\ \mathrm{H} & 4.87803 & 0.22546 & -1.30021 \\ \mathrm{H} & -1.07414 & -0.79728 & -1.55644 \\ \mathrm{H} & -2.97762 & -4.08064 & -1.54175\end{array}$




\begin{tabular}{|c|c|c|c|c|c|c|c|}
\hline $\mathrm{C}$ & 6.45420 & 0.08053 & 0.18763 & $\mathrm{H}$ & 7.43761 & -1.97221 & -3.00376 \\
\hline $\mathrm{C}$ & 3.65042 & 0.02518 & 1.27246 & $\mathrm{H}$ & 3.66144 & 1.64051 & -0.20908 \\
\hline $\mathrm{C}$ & -5.77795 & -0.52511 & -1.82752 & $\mathrm{H}$ & 2.69168 & 1.97786 & 1.22341 \\
\hline $\mathrm{C}$ & 1.47344 & -0.03867 & 2.77732 & $\mathrm{H}$ & 2.08396 & 0.87387 & -0.01846 \\
\hline $\mathrm{C}$ & 4.81663 & 0.53156 & 2.12997 & $\mathrm{H}$ & 1.68311 & -2.64854 & 1.21925 \\
\hline $\mathrm{C}$ & 5.94438 & 1.07970 & 1.24102 & $\mathrm{H}$ & -4.99262 & -1.46968 & -0.04454 \\
\hline $\mathrm{C}$ & -0.31581 & -1.92665 & 0.09725 & $\mathrm{H}$ & -7.37617 & -0.64257 & -0.39857 \\
\hline $\mathrm{C}$ & 5.30289 & -0.53759 & -0.63145 & $\mathrm{H}$ & 9.98310 & 2.17201 & -1.16902 \\
\hline $\mathrm{C}$ & -1.48236 & 1.18839 & -0.17430 & $\mathrm{H}$ & 10.97341 & 1.28165 & -0.01257 \\
\hline $\mathrm{C}$ & 7.88962 & -0.27618 & -1.87051 & $\mathrm{H}$ & 6.15842 & 2.00000 & -1.73139 \\
\hline $\mathrm{C}$ & -1.43982 & -1.15694 & -0.59251 & $\mathrm{H}$ & 7.84648 & 2.37510 & -2.07592 \\
\hline $\mathrm{C}$ & -2.17054 & -3.33614 & -1.56719 & $\mathrm{H}$ & 7.16113 & 2.81507 & -0.51433 \\
\hline $\mathrm{C}$ & 7.12350 & -1.32397 & -2.18547 & $\mathrm{H}$ & 8.69173 & 1.59993 & 0.90410 \\
\hline $\mathrm{C}$ & 2.98346 & 1.18901 & 0.51925 & $\mathrm{H}$ & 9.04679 & -0.10886 & 0.66083 \\
\hline $\mathrm{C}$ & 1.92852 & -1.65688 & 0.82652 & $\mathrm{H}$ & -6.75321 & -3.06886 & -0.04266 \\
\hline $\mathrm{C}$ & 7.57328 & 0.67371 & -0.71532 & $\mathrm{H}$ & -6.83640 & -4.19677 & -1.41920 \\
\hline $\mathrm{C}$ & -4.90626 & -1.57292 & -1.14193 & $\mathrm{H}$ & -1.17355 & -4.38087 & 0.03643 \\
\hline $\mathrm{C}$ & -7.23842 & -0.77831 & -1.48327 & $\mathrm{H}$ & -0.50935 & -4.70247 & -1.58728 \\
\hline $\mathrm{C}$ & 10.10360 & 1.19981 & -0.67356 & $\mathrm{H}$ & -5.62346 & 2.54357 & 0.26647 \\
\hline $\mathrm{C}$ & 7.16079 & 2.04395 & -1.29155 & $\mathrm{H}$ & 10.55140 & -0.81867 & -1.24113 \\
\hline $\mathrm{C}$ & 8.85819 & 0.83789 & 0.13537 & $\mathrm{H}$ & 9.06388 & 0.96172 & -3.20224 \\
\hline $\mathrm{C}$ & -6.64105 & -3.16236 & -1.13334 & $\mathrm{H}$ & 9.32057 & -0.77432 & -3.39364 \\
\hline $\mathrm{C}$ & -0.91997 & -3.93055 & -0.93441 & $\mathrm{H}$ & -7.51846 & -2.34221 & -2.91721 \\
\hline $\mathrm{C}$ & -4.90595 & 3.20427 & -0.20791 & $\mathrm{H}$ & 2.58644 & 1.60611 & 3.66759 \\
\hline $\mathrm{C}$ & 10.37176 & 0.14956 & -1.73710 & $\mathrm{H}$ & 2.71248 & 0.07266 & 4.55501 \\
\hline $\mathrm{C}$ & -4.27301 & 2.80701 & -1.38597 & $\mathrm{H}$ & 1.23538 & 1.05393 & 4.65677 \\
\hline $\mathrm{C}$ & -4.56466 & 1.51285 & -2.04656 & $\mathrm{H}$ & -5.13532 & 4.72919 & 1.28041 \\
\hline $\mathrm{C}$ & 9.15258 & 0.01493 & -2.65073 & $\mathrm{H}$ & -0.95559 & 0.57200 & 3.84283 \\
\hline $\mathrm{C}$ & -7.61002 & -2.21299 & -1.82762 & $\mathrm{H}$ & -1.34715 & -0.28314 & 2.36923 \\
\hline $\mathrm{C}$ & 2.03670 & 0.71960 & 3.99018 & $\mathrm{H}$ & -2.84387 & 3.34212 & -2.89936 \\
\hline $\mathrm{C}$ & -4.62932 & 4.44036 & 0.36741 & $\mathrm{H}$ & -2.33855 & 5.56961 & -1.87858 \\
\hline $\mathrm{C}$ & -1.00736 & -0.43386 & 3.40364 & $\mathrm{H}$ & -1.62022 & -1.40444 & 5.21503 \\
\hline $\mathrm{C}$ & -3.34120 & 3.66271 & -1.98999 & $\mathrm{H}$ & -1.92517 & -2.34636 & 3.76462 \\
\hline $\mathrm{C}$ & -3.70393 & 5.29158 & -0.24732 & $\mathrm{H}$ & -3.42421 & 0.23218 & 4.41242 \\
\hline $\mathrm{C}$ & -3.05618 & 4.89204 & -1.42820 & $\mathrm{H}$ & -4.24217 & -2.66307 & 4.98408 \\
\hline $\mathrm{C}$ & -1.96739 & -1.32942 & 4.17678 & $\mathrm{H}$ & -5.28782 & -1.24935 & 5.19694 \\
\hline $\mathrm{C}$ & -3.41977 & -0.83691 & 4.15386 & $\mathrm{H}$ & -3.84109 & -1.44293 & 6.20494 \\
\hline $\mathrm{C}$ & -4.24649 & -1.58730 & 5.19833 & $\mathrm{H}$ & -5.08059 & -0.62756 & 2.76111 \\
\hline $\mathrm{C}$ & -4.05107 & -1.00225 & 2.76855 & $\mathrm{H}$ & -4.08238 & -2.06700 & 2.49929 \\
\hline $\mathrm{C}$ & -3.98519 & 6.96166 & 1.41524 & $\mathrm{H}$ & -3.49544 & -0.47356 & 1.98654 \\
\hline $\mathrm{H}$ & 2.53222 & -2.24366 & 3.27916 & $\mathrm{H}$ & -3.57615 & 7.95231 & 1.60794 \\
\hline $\mathrm{H}$ & -2.60968 & -2.44112 & -3.21350 & $\mathrm{H}$ & -3.74780 & 6.29683 & 2.25228 \\
\hline $\mathrm{H}$ & -8.01875 & 0.96391 & -1.86105 & $\mathrm{H}$ & -5.07133 & 7.02943 & 1.29537 \\
\hline
\end{tabular}


Structure, energy and Cartesian coordinate of 1-Bc:

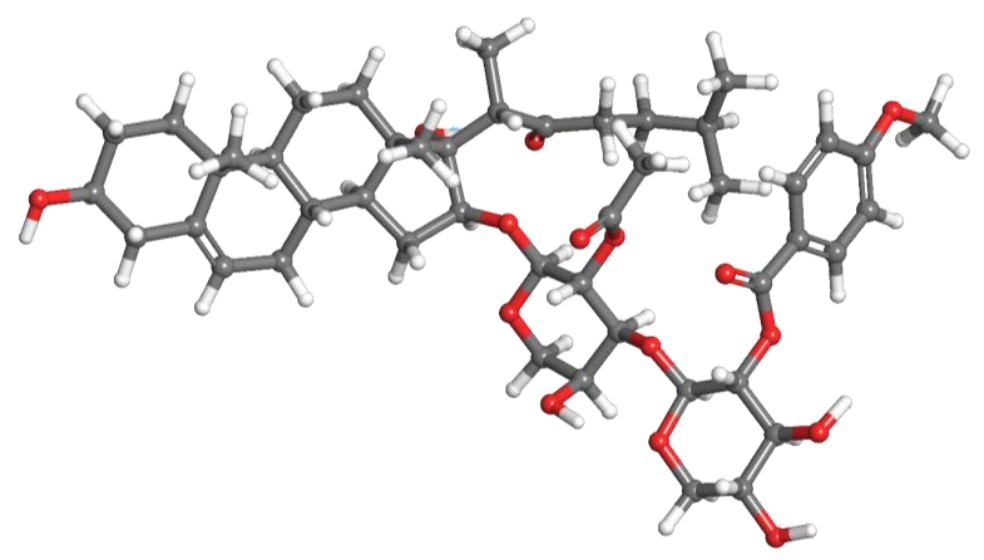

1-Bc $(\Delta G=0.6 \mathrm{kcal} / \mathrm{mol}$, Boltzmann pop. $(298 \mathrm{~K})=9.1 \%)$

M06-2X/6-311+G(d,p)-PCM(MeOH)//M06-2X/6-31G(d)-PCM(MeOH):

\begin{tabular}{rrr} 
& Gibbs Free Energy (a.u. $)=$ & -2958.9934836 \\
\hline M06-2X/6-311+G(d,p)-PCM(MeOH): & Electronic energy (a.u. $)=$ & -2960.0332836 \\
\hline M06-2X/6-31G(d)-PCM(MeOH): & Zero-point correction $($ a.u. $)=$ & 1.134711
\end{tabular}

Thermal correction to Energy (a.u.) $=1.194915$

Thermal correction to Enthalpy (a.u.) $=1.195859$

Thermal correction to Gibbs Free Energy (a.u.) $=1.039800$

$\begin{array}{lrrrrrrr}\mathrm{O} & -0.92786 & -0.28476 & 0.70904 & \mathrm{H} & 7.42100 & 6.05072 & -0.40827 \\ \mathrm{O} & 2.95568 & 2.23284 & 0.05734 & \mathrm{H} & -11.98367 & 2.79608 & -0.64176 \\ \mathrm{O} & 5.67169 & 1.61480 & -0.64335 & \mathrm{H} & 2.85075 & -1.75167 & -1.73528 \\ \mathrm{O} & 1.62571 & -0.14114 & -0.34034 & \mathrm{H} & 2.26549 & -1.18533 & -3.33282 \\ \mathrm{O} & -3.34534 & -2.57995 & 2.07007 & \mathrm{H} & 1.21490 & -2.16700 & -2.28832 \\ \mathrm{O} & -0.48968 & 1.44691 & 2.14030 & \mathrm{H} & 2.77568 & 1.01086 & 1.71505 \\ \mathrm{O} & 0.90202 & 3.75878 & 1.22126 & \mathrm{H} & -3.33744 & 1.08529 & 2.16644 \\ \mathrm{O} & 3.58090 & 4.35177 & 0.54093 & \mathrm{H} & -2.85572 & 1.16734 & 0.47061 \\ \mathrm{O} & 7.15162 & 3.96789 & -1.44340 & \mathrm{H} & -6.16690 & 1.23044 & 2.08843 \\ \mathrm{O} & 6.68597 & 6.18775 & 0.21170 & \mathrm{H} & -5.30541 & 2.41015 & 1.12029 \\ \mathrm{O} & -11.83186 & 1.96738 & -1.12010 & \mathrm{H} & -4.87447 & -0.72828 & 1.74440 \\ \mathrm{O} & -0.70681 & -3.09496 & 2.52854 & \mathrm{H} & -7.13657 & -0.77634 & 0.79604 \\ \mathrm{O} & 0.82457 & 0.71685 & -2.27013 & \mathrm{H} & 4.62976 & 3.11135 & -1.63496 \\ \mathrm{O} & 4.31537 & 0.87700 & -2.29635 & \mathrm{H} & -1.23199 & -2.38773 & -0.54624 \\ \mathrm{O} & 7.54094 & -4.39257 & -0.86989 & \mathrm{H} & -5.38046 & -2.82114 & 0.80196 \\ \mathrm{C} & 1.98886 & -1.39143 & -2.29882 & \mathrm{H} & -4.76571 & -3.32211 & -0.77287 \\ \mathrm{C} & 2.16405 & 1.69529 & 1.10742 & \mathrm{H} & -7.06359 & -2.46081 & -0.97003 \\ \mathrm{C} & -3.18959 & 0.48743 & 1.26323 & \mathrm{H} & -5.93032 & -1.51459 & -1.91098 \\ \mathrm{C} & -2.73718 & -1.94647 & 0.95213 & \mathrm{H} & 0.55361 & -0.32948 & 2.13486 \\ \mathrm{C} & -6.05727 & 1.61274 & 1.06069 & \mathrm{H} & -5.18152 & 0.94449 & -0.77267 \\ \mathrm{C} & -4.43719 & -0.28600 & 0.83672 & \mathrm{H} & 0.51339 & 1.59962 & -0.25301 \\ \mathrm{C} & -0.51925 & -3.08386 & 1.32000 & \mathrm{H} & 2.31906 & 3.22063 & 2.64158\end{array}$




\begin{tabular}{|c|c|c|c|}
\hline $\mathrm{C}$ & -6.71979 & -0.46997 & -0.17916 \\
\hline $\mathrm{C}$ & -3.89237 & -1.47898 & 0.01957 \\
\hline $\mathrm{C}$ & 5.09509 & 2.91811 & -0.66432 \\
\hline $\mathrm{C}$ & -1.66325 & -2.86854 & 0.33682 \\
\hline $\mathrm{C}$ & -5.06100 & -2.45085 & -0.17822 \\
\hline $\mathrm{C}$ & -6.23293 & -1.75095 & -0.88489 \\
\hline $\mathrm{C}$ & 0.06429 & 0.36750 & 1.42370 \\
\hline $\mathrm{C}$ & -5.55845 & 0.48799 & 0.15429 \\
\hline $\mathrm{C}$ & 1.42317 & -0.14651 & -1.67889 \\
\hline $\mathrm{C}$ & -8.16308 & 1.60630 & -0.30977 \\
\hline $\mathrm{C}$ & 1.05779 & 0.92517 & 0.41031 \\
\hline $\mathrm{C}$ & 1.53976 & 2.77667 & 2.00389 \\
\hline $\mathrm{C}$ & -7.36365 & 2.18218 & 0.59246 \\
\hline $\mathrm{C}$ & -3.30490 & -1.04674 & -1.33398 \\
\hline $\mathrm{C}$ & -2.10991 & -0.59744 & 1.45616 \\
\hline $\mathrm{C}$ & -7.87233 & 0.24456 & -0.94182 \\
\hline $\mathrm{C}$ & 4.03316 & 3.01153 & 0.42919 \\
\hline $\mathrm{C}$ & 6.22297 & 3.89996 & -0.38315 \\
\hline $\mathrm{C}$ & -10.42029 & 0.07089 & -1.33848 \\
\hline $\mathrm{C}$ & -7.52012 & 0.44530 & -2.43006 \\
\hline $\mathrm{C}$ & -9.15422 & -0.61879 & -0.82851 \\
\hline $\mathrm{C}$ & 4.60680 & 5.26523 & 0.90824 \\
\hline $\mathrm{C}$ & 0.49024 & 2.13558 & 2.90317 \\
\hline $\mathrm{C}$ & 5.54023 & -1.68460 & -2.15339 \\
\hline $\mathrm{C}$ & -10.65767 & 1.37067 & -0.58996 \\
\hline $\mathrm{C}$ & 5.87729 & -0.62764 & -1.29709 \\
\hline $\mathrm{C}$ & 5.18967 & 0.67134 & -1.48563 \\
\hline $\mathrm{C}$ & -9.44318 & 2.28679 & -0.74241 \\
\hline $\mathrm{C}$ & 5.66637 & 5.30164 & -0.18526 \\
\hline $\mathrm{C}$ & -2.20755 & -4.25016 & -0.06047 \\
\hline $\mathrm{C}$ & 6.11600 & -2.92857 & -1.99007 \\
\hline $\mathrm{C}$ & 0.86050 & -3.29679 & 0.74282 \\
\hline $\mathrm{C}$ & 6.82996 & -0.83318 & -0.29899 \\
\hline $\mathrm{C}$ & 7.05515 & -3.13717 & -0.96740 \\
\hline $\mathrm{C}$ & 7.42710 & -2.07919 & -0.13071 \\
\hline $\mathrm{C}$ & 1.84947 & -3.88590 & 1.74477 \\
\hline $\mathrm{C}$ & 3.31306 & -3.87819 & 1.28248 \\
\hline $\mathrm{C}$ & 3.87836 & -2.45925 & 1.20357 \\
\hline $\mathrm{C}$ & 3.51233 & -4.62068 & -0.04002 \\
\hline $\mathrm{C}$ & 8.46809 & -4.66706 & 0.16998 \\
\hline $\mathrm{H}$ & -2.60614 & -2.84069 & 2.65012 \\
\hline $\mathrm{H}$ & 1.61131 & 4.21211 & 0.73171 \\
\hline $\mathrm{H}$ & 7.64314 & 3.13279 & -1.46914 \\
\hline
\end{tabular}

\begin{tabular}{|c|c|c|c|}
\hline $\mathrm{H}$ & -7.66496 & 3.13907 & 1.01924 \\
\hline 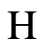 & -4.02093 & -0.46879 & -1.92279 \\
\hline $\mathrm{H}$ & -3.03526 & -1.92815 & -1.92792 \\
\hline $\mathrm{H}$ & -2.40354 & -0.43682 & -1.21648 \\
\hline & -1.83353 & -0.72163 & 2.50866 \\
\hline $\mathrm{H}$ & 4.45671 & 2.68264 & 1.39474 \\
\hline $\mathrm{H}$ & 6.72378 & 3.59996 & 0.55124 \\
\hline $\mathrm{H}$ & -10.34008 & 0.29720 & -2.40983 \\
\hline $\mathrm{H}$ & -11.28651 & -0.58804 & -1.21332 \\
\hline $\mathrm{H}$ & -6.51974 & 0.87677 & -2.54437 \\
\hline $\mathrm{H}$ & -8.22389 & 1.12110 & -2.92307 \\
\hline $\mathbf{H}$ & -7.54847 & -0.50506 & -2.97261 \\
\hline $\mathrm{H}$ & -9.00826 & -1.55913 & -1.37118 \\
\hline $\mathrm{H}$ & -9.30085 & -0.88081 & 0.22885 \\
\hline $\mathrm{H}$ & 5.06578 & 4.96959 & 1.86301 \\
\hline $\mathrm{H}$ & 4.13450 & 6.24044 & 1.03049 \\
\hline H & 0.97090 & 1.45009 & 3.61651 \\
\hline $\mathrm{H}$ & -0.03961 & 2.91188 & 3.45699 \\
\hline $\mathbf{H}$ & 4.82176 & -1.51090 & -2.94758 \\
\hline $\mathrm{H}$ & -10.79685 & 1.14228 & 0.47981 \\
\hline $\mathrm{H}$ & -9.39536 & 2.57774 & -1.80147 \\
\hline $\mathrm{H}$ & -9.58863 & 3.20717 & -0.16383 \\
\hline $\mathrm{H}$ & 5.19759 & 5.62688 & -1.12685 \\
\hline $\mathrm{H}$ & -2.82365 & -4.17656 & -0.95931 \\
\hline $\mathrm{H}$ & -2.81486 & -4.66339 & 0.74880 \\
\hline $\mathrm{H}$ & -1.39338 & -4.94949 & -0.27680 \\
\hline $\mathbf{H}$ & 5.85852 & -3.76221 & -2.63487 \\
\hline $\mathrm{H}$ & 0.77201 & -3.91235 & -0.16168 \\
\hline $\mathbf{H}$ & 1.18043 & -2.30228 & 0.39442 \\
\hline $\mathrm{H}$ & 7.10372 & -0.01756 & 0.36096 \\
\hline $\mathrm{H}$ & 8.16039 & -2.21722 & 0.65431 \\
\hline $\mathrm{H}$ & 1.54441 & -4.91537 & 1.97230 \\
\hline $\mathrm{H}$ & 1.76812 & -3.32472 & 2.68260 \\
\hline $\mathrm{H}$ & 3.88152 & -4.41665 & 2.05318 \\
\hline $\mathrm{H}$ & 3.35061 & -1.85165 & 0.45881 \\
\hline $\mathrm{H}$ & 4.93574 & -2.47977 & 0.92040 \\
\hline $\mathrm{H}$ & 3.79088 & -1.94717 & 2.16922 \\
\hline 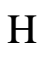 & 4.57873 & -4.73422 & -0.26378 \\
\hline $\mathrm{H}$ & 3.05999 & -4.07120 & -0.87514 \\
\hline $\mathrm{H}$ & 3.06055 & -5.61883 & -0.00938 \\
\hline $\mathbf{H}$ & 8.71228 & -5.72458 & 0.08170 \\
\hline $\mathrm{H}$ & 8.02233 & -4.47254 & 1.15114 \\
\hline $\mathrm{H}$ & 9.37799 & -4.06915 & 0.05378 \\
\hline
\end{tabular}


Structure, energy and Cartesian coordinate of 1-Bd:

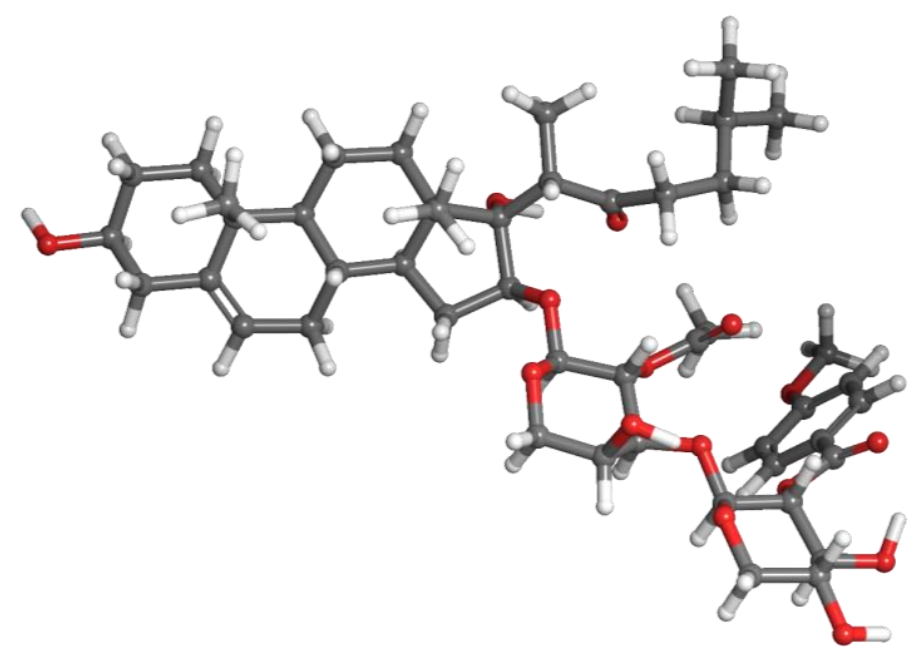

1-Bd $(\Delta G=1.2 \mathrm{kcal} / \mathrm{mol}$, Boltzmann pop. $(298 \mathrm{~K})=4.1 \%)$

M06-2X/6-311+G(d,p)-PCM(MeOH)//M06-2X/6-31G(d)-PCM(MeOH):

Gibbs Free Energy (a.u.) $=-2958.9927315$

M06-2X/6-311+G(d,p)-PCM(MeOH): Electronic energy (a.u. $)=-2960.0315295$

M06-2X/6-31G(d)-PCM(MeOH): Zero-point correction $($ a.u. $)=1.135023$

Thermal correction to Energy (a.u.) $=1.195239$

Thermal correction to Enthalpy (a.u.) $=1.196183$

Thermal correction to Gibbs Free Energy (a.u.) $=1.038798$

$\begin{array}{lrrrrrrr}\mathrm{O} & 0.85888 & -0.21290 & -0.80837 & \mathrm{H} & -8.62529 & -4.85877 & -0.59006 \\ \mathrm{O} & -3.44302 & -2.09209 & -0.56147 & \mathrm{H} & 12.34685 & -1.27454 & 2.39596 \\ \mathrm{O} & -5.67615 & -1.28722 & 0.83476 & \mathrm{H} & -2.64108 & 1.53183 & 2.00161 \\ \mathrm{O} & -1.66615 & -0.05118 & 0.36322 & \mathrm{H} & -3.68702 & 2.44182 & 0.86583 \\ \mathrm{O} & 2.76524 & 2.55372 & 0.35528 & \mathrm{H} & -1.91681 & 2.67880 & 0.85414 \\ \mathrm{O} & 0.60313 & -2.43316 & -1.11467 & \mathrm{H} & -2.05937 & -2.51622 & 0.91787 \\ \mathrm{O} & -1.80004 & -3.50444 & -2.27746 & \mathrm{H} & 2.64510 & -0.94854 & 1.67421 \\ \mathrm{O} & -4.43744 & -4.03612 & -1.16820 & \mathrm{H} & 2.80086 & -1.56327 & 0.02218 \\ \mathrm{O} & -8.15603 & -2.62946 & 0.03974 & \mathrm{H} & 5.26830 & -0.75488 & 2.68704 \\ \mathrm{O} & -7.79122 & -5.34203 & -0.71260 & \mathrm{H} & 4.82565 & -2.27823 & 1.94264 \\ \mathrm{O} & 11.65549 & -1.93321 & 2.23206 & \mathrm{H} & 4.23677 & 0.82471 & 1.26307 \\ \mathrm{O} & 0.12715 & 2.87525 & -0.37098 & \mathrm{H} & 6.69219 & 0.82905 & 1.20682 \\ \mathrm{O} & -3.08232 & 0.87231 & -1.13093 & \mathrm{H} & -5.84903 & -1.78355 & -1.16300 \\ \mathrm{O} & -7.08666 & 0.01566 & -0.33854 & \mathrm{H} & 1.75276 & 1.24464 & -2.57677 \\ \mathrm{O} & -5.21365 & 3.80346 & 4.44028 & \mathrm{H} & 5.12729 & 2.54645 & -0.11238 \\ \mathrm{C} & -2.72166 & 1.95043 & 0.99815 & \mathrm{H} & 5.13262 & 2.42950 & -1.87199 \\ \mathrm{C} & -2.10464 & -2.36241 & -0.17114 & \mathrm{H} & 7.32583 & 1.78939 & -0.95800 \\ \mathrm{C} & 2.83487 & -0.65572 & 0.63771 & \mathrm{H} & 6.57782 & 0.46332 & -1.82412 \\ \mathrm{C} & 2.58721 & 1.50822 & -0.59035 & \mathrm{H} & 0.39240 & -1.51258 & 0.71894 \\ \mathrm{C} & 5.55241 & -1.45740 & 1.88685 & \mathrm{H} & 5.43218 & -1.52409 & -0.24939\end{array}$




\begin{tabular}{|c|c|c|c|c|c|c|c|}
\hline $\mathrm{C}$ & 4.16748 & 0.07315 & 0.46198 & $\mathrm{H}$ & -1.52521 & -0.82855 & -1.56460 \\
\hline $\mathrm{C}$ & 0.42388 & 2.46356 & -1.48414 & $\mathrm{H}$ & -1.96494 & -4.50077 & -0.46456 \\
\hline $\mathrm{C}$ & 6.66605 & 0.16797 & 0.32334 & $\mathrm{H}$ & 7.03343 & -2.70761 & 2.96122 \\
\hline $\mathrm{C}$ & 3.98692 & 0.87508 & -0.84388 & $\mathrm{H}$ & 4.76776 & -0.71569 & -2.13459 \\
\hline $\mathrm{C}$ & -5.74184 & -2.27517 & -0.18991 & $\mathrm{H}$ & 3.90694 & 0.55638 & -2.99645 \\
\hline $\mathrm{C}$ & 1.84084 & 2.03962 & -1.83119 & $\mathrm{H}$ & 3.00399 & -0.65497 & -2.07400 \\
\hline $\mathrm{C}$ & 5.17256 & 1.84063 & -0.94926 & $\mathrm{H}$ & 1.21632 & 0.79770 & 0.94042 \\
\hline $\mathrm{C}$ & 6.50108 & 1.06927 & -0.91434 & $\mathrm{H}$ & -4.19195 & -3.44629 & 0.80067 \\
\hline $\mathrm{C}$ & 0.17964 & -1.33153 & -0.34888 & $\mathrm{H}$ & -6.76372 & -3.69882 & 1.04574 \\
\hline $\mathrm{C}$ & 5.44685 & -0.75246 & 0.53423 & $\mathrm{H}$ & 10.84160 & -0.87368 & -0.05609 \\
\hline $\mathrm{C}$ & -2.54899 & 0.88475 & -0.04705 & $\mathrm{H}$ & 11.28738 & 0.47078 & 0.99621 \\
\hline $\mathrm{C}$ & 8.02108 & -1.64892 & 1.45582 & $\mathrm{H}$ & 7.33817 & -1.90976 & -1.31200 \\
\hline $\mathrm{C}$ & -1.31833 & -1.10961 & -0.52886 & $\mathrm{H}$ & 9.05751 & -2.06077 & -0.95431 \\
\hline $\mathrm{C}$ & -1.53001 & -3.58813 & -0.89685 & $\mathrm{H}$ & 8.47082 & -0.63845 & -1.81433 \\
\hline $\mathrm{C}$ & 6.93093 & -1.98766 & 2.14914 & $\mathrm{H}$ & 9.25182 & 1.05783 & -0.28479 \\
\hline $\mathrm{C}$ & 3.91022 & -0.04089 & -2.07718 & $\mathrm{H}$ & 8.91695 & 0.99259 & 1.44422 \\
\hline $\mathrm{C}$ & 1.78956 & 0.35581 & 0.11643 & $\mathrm{H}$ & -5.29219 & -5.48260 & 0.06251 \\
\hline $\mathrm{C}$ & 8.00755 & -0.61950 & 0.32502 & $\mathrm{H}$ & -5.32831 & -5.78102 & -1.69322 \\
\hline $\mathrm{C}$ & -4.40504 & -3.01007 & -0.19078 & $\mathrm{H}$ & 0.20976 & -3.84556 & 0.35414 \\
\hline $\mathrm{C}$ & -6.88481 & -3.24546 & 0.05170 & $\mathrm{H}$ & 0.40097 & -4.43339 & -1.31835 \\
\hline $\mathrm{C}$ & 10.52057 & -0.29387 & 0.81902 & $\mathrm{H}$ & -7.13016 & 2.38616 & 0.54221 \\
\hline $\mathrm{C}$ & 8.23080 & -1.34812 & -1.01610 & $\mathrm{H}$ & 10.17170 & -0.64799 & 2.90977 \\
\hline $\mathrm{C}$ & 9.16900 & 0.37699 & 0.56928 & $\mathrm{H}$ & 9.70104 & -2.91436 & 0.94881 \\
\hline $\mathrm{C}$ & -5.43959 & -5.01738 & -0.92283 & $\mathrm{H}$ & 9.26730 & -2.92605 & 2.65860 \\
\hline $\mathrm{C}$ & -0.02156 & -3.63857 & -0.70144 & $\mathrm{H}$ & -6.95672 & -3.92738 & -1.98541 \\
\hline $\mathrm{C}$ & -6.54808 & 2.17563 & 1.43364 & $\mathrm{H}$ & 3.41493 & 2.95299 & -3.03342 \\
\hline $\mathrm{C}$ & 10.43868 & -1.23429 & 2.01488 & $\mathrm{H}$ & 2.82845 & 3.96749 & -1.69749 \\
\hline $\mathrm{C}$ & -6.06309 & 0.88372 & 1.63749 & $\mathrm{H}$ & 1.85843 & 3.76868 & -3.17164 \\
\hline $\mathrm{C}$ & -6.34638 & -0.14156 & 0.61319 & $\mathrm{H}$ & -6.65897 & 4.18790 & 2.16100 \\
\hline $\mathrm{C}$ & 9.35701 & -2.28040 & 1.77871 & $\mathrm{H}$ & -0.09511 & 2.44421 & -3.56338 \\
\hline $\mathrm{C}$ & -6.81506 & -4.36463 & -0.98436 & $\mathrm{H}$ & -1.02968 & 1.38315 & -2.52834 \\
\hline $\mathrm{C}$ & 2.52909 & 3.25713 & -2.47211 & $\mathrm{H}$ & -4.93035 & -0.40163 & 2.95002 \\
\hline $\mathrm{C}$ & -6.28121 & 3.18977 & 2.34555 & $\mathrm{H}$ & -4.48089 & 1.39805 & 4.60858 \\
\hline $\mathrm{C}$ & -0.60307 & 2.39409 & -2.59401 & $\mathrm{H}$ & -2.13649 & 3.36387 & -1.45940 \\
\hline $\mathrm{C}$ & -5.31296 & 0.60015 & 2.78766 & $\mathrm{H}$ & -2.50999 & 3.21123 & -3.16999 \\
\hline $\mathrm{C}$ & -5.53013 & 2.89906 & 3.49170 & $\mathrm{H}$ & -0.33076 & 5.05002 & -2.07869 \\
\hline $\mathrm{C}$ & -5.05635 & 1.59598 & 3.71053 & $\mathrm{H}$ & -3.21941 & 5.71366 & -2.84306 \\
\hline $\mathrm{C}$ & -1.70365 & 3.44298 & -2.46214 & $\mathrm{H}$ & -1.97762 & 6.90614 & -2.42577 \\
\hline $\mathrm{C}$ & -1.22273 & 4.88001 & -2.70073 & $\mathrm{H}$ & -2.54500 & 5.75058 & -1.20461 \\
\hline $\mathrm{C}$ & -2.30058 & 5.87237 & -2.26500 & $\mathrm{H}$ & -0.49017 & 6.14247 & -4.31438 \\
\hline $\mathrm{C}$ & -0.84862 & 5.11891 & -4.16467 & $\mathrm{H}$ & -1.72621 & 4.97197 & -4.80641 \\
\hline $\mathrm{C}$ & -5.64691 & 5.14313 & 4.25427 & $\mathrm{H}$ & -0.06458 & 4.43910 & -4.51393 \\
\hline $\mathrm{H}$ & 1.87002 & 2.90492 & 0.51715 & $\mathrm{H}$ & -5.27627 & 5.69918 & 5.11409 \\
\hline $\mathrm{H}$ & -2.76871 & -3.54433 & -2.35927 & $\mathrm{H}$ & -6.73973 & 5.20175 & 4.22252 \\
\hline $\mathrm{H}$ & -8.10296 & -1.82550 & -0.50581 & $\mathrm{H}$ & -5.22893 & 5.56495 & 3.33448 \\
\hline
\end{tabular}


Structure, energy and Cartesian coordinate of 1-Be:

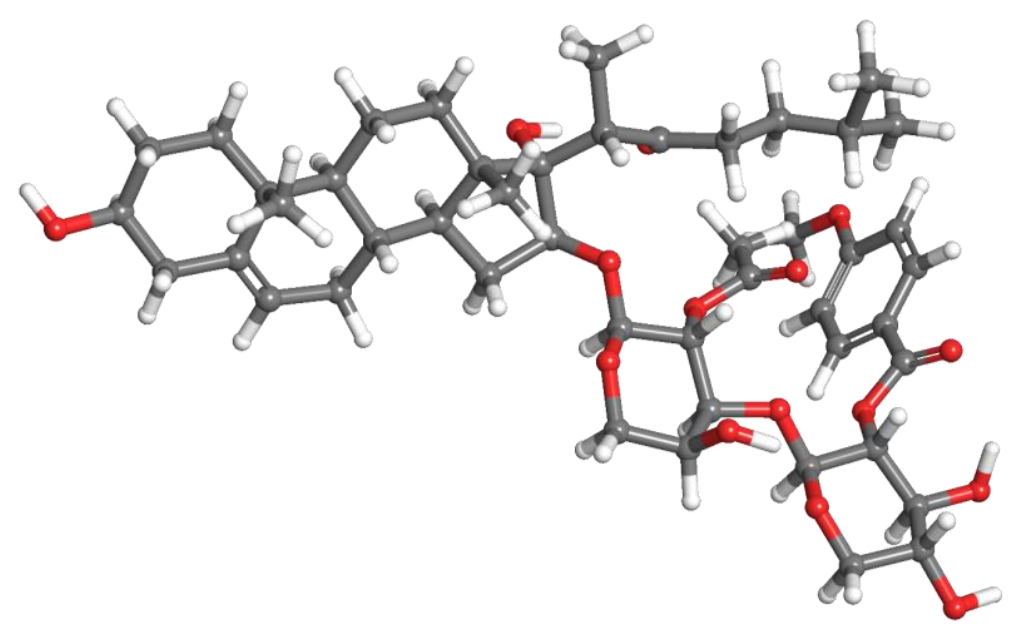

1-Be $(\Delta G=1.4 \mathrm{kcal} / \mathrm{mol}$, Boltzmann pop. $(298 \mathrm{~K})=3.3 \%)$

M06-2X/6-311+G(d,p)-PCM(MeOH)//M06-2X/6-31G(d)-PCM(MeOH):

Gibbs Free Energy (a.u.) = -2958.9925293

M06-2X/6-311+G(d,p)-PCM(MeOH): $\quad$ Electronic energy (a.u. $)=-2960.0315433$

M06-2X/6-31G(d)-PCM(MeOH): Zero-point correction (a.u.) $=1.134552$

Thermal correction to Energy (a.u.) $=1.194821$

Thermal correction to Enthalpy (a.u.) $=1.195765$

Thermal correction to Gibbs Free Energy (a.u.) = 1.039014

$\begin{array}{lrrrrrrr}\mathrm{O} & -0.95264 & 0.10547 & -0.56619 & \mathrm{H} & 8.38011 & 4.97965 & -0.38870 \\ \mathrm{O} & 3.23967 & 2.15675 & -0.25638 & \mathrm{H} & -12.64599 & 0.88065 & 1.87278 \\ \mathrm{O} & 5.60633 & 1.17744 & 0.75184 & \mathrm{H} & 2.75833 & -1.56085 & 2.25479 \\ \mathrm{O} & 1.55610 & -0.00594 & 0.64826 & \mathrm{H} & 3.57845 & -2.54809 & 1.00552 \\ \mathrm{O} & -2.89867 & -2.72402 & 0.36365 & \mathrm{H} & 1.79770 & -2.60691 & 1.19000 \\ \mathrm{O} & -0.79769 & 2.35533 & -0.71460 & \mathrm{H} & 1.91068 & 2.41794 & 1.31240 \\ \mathrm{O} & 1.52721 & 3.65137 & -1.78020 & \mathrm{H} & -2.91335 & 0.71508 & 1.82080 \\ \mathrm{O} & 4.14518 & 4.20899 & -0.58893 & \mathrm{H} & -2.96736 & 1.39815 & 0.18943 \\ \mathrm{O} & 7.96868 & 2.64817 & -0.11395 & \mathrm{H} & -5.58755 & 0.46027 & 2.63846 \\ \mathrm{O} & 7.53974 & 5.46342 & -0.32707 & \mathrm{H} & -5.12390 & 2.01661 & 1.98060 \\ \mathrm{O} & -11.95346 & 1.55593 & 1.81675 & \mathrm{H} & -4.45055 & -1.05648 & 1.22538 \\ \mathrm{O} & -0.21283 & -2.99884 & -0.18095 & \mathrm{H} & -6.88954 & -1.08722 & 0.99915 \\ \mathrm{O} & 3.11498 & -0.75555 & -0.80280 & \mathrm{H} & 5.53088 & 2.00637 & -1.13606 \\ \mathrm{O} & 6.73121 & 0.07420 & -0.85617 & \mathrm{H} & -1.69442 & -1.30322 & -2.44185 \\ \mathrm{O} & 5.83042 & -4.51710 & 3.42863 & \mathrm{H} & -5.22102 & -2.72781 & -0.28396 \\ \mathrm{C} & 2.68550 & -1.96845 & 1.24467 & \mathrm{H} & -5.10659 & -2.52902 & -2.03296 \\ \mathrm{C} & 1.91427 & 2.34362 & 0.21442 & \mathrm{H} & -7.36339 & -1.95403 & -1.24670 \\ \mathrm{C} & -3.02813 & 0.46511 & 0.76217 & \mathrm{H} & -6.57473 & -0.58232 & -1.99719 \\ \mathrm{C} & -2.66573 & -1.64451 & -0.53052 & \mathrm{H} & -0.56425 & 1.31666 & 1.05146 \\ \mathrm{C} & -5.83215 & 1.18853 & 1.84827 & \mathrm{H} & -5.56884 & 1.33977 & -0.27091 \\ \mathrm{C} & -4.33482 & -0.27091 & 0.46370 & \mathrm{H} & 1.40036 & 0.84827 & -1.24531\end{array}$




\begin{tabular}{|c|c|c|c|c|c|c|c|}
\hline $\mathrm{C}$ & -0.43012 & -2.54434 & -1.29554 & $\mathrm{H}$ & 1.66067 & 4.48792 & 0.11328 \\
\hline $\mathrm{C}$ & -6.81572 & -0.38596 & 0.14984 & $\mathrm{H}$ & -7.39990 & 2.37180 & 2.87396 \\
\hline $\mathrm{C}$ & -4.05150 & -1.01477 & -0.85865 & $\mathrm{H}$ & -4.75541 & 0.62412 & -2.13299 \\
\hline $\mathrm{C}$ & 5.54648 & 2.32498 & -0.08831 & $\mathrm{H}$ & -3.82435 & -0.60521 & -2.98498 \\
\hline $\mathrm{C}$ & -1.82546 & -2.12513 & -1.73242 & $\mathrm{H}$ & -2.99944 & 0.57280 & -1.95263 \\
\hline $\mathrm{C}$ & -5.21629 & -1.98457 & -1.08895 & $\mathrm{H}$ & -1.40951 & -0.97682 & 1.11397 \\
\hline $\mathrm{C}$ & -6.55383 & -1.22793 & -1.11230 & $\mathrm{H}$ & 4.09863 & 3.26658 & 1.25408 \\
\hline $\mathrm{C}$ & -0.33279 & 1.22085 & -0.02375 & $\mathrm{H}$ & 6.74480 & 3.52191 & 1.23273 \\
\hline $\mathrm{C}$ & -5.62690 & 0.53797 & 0.47976 & $\mathrm{H}$ & -10.97294 & 0.61162 & -0.45641 \\
\hline $\mathrm{C}$ & 2.51778 & -0.86236 & 0.24148 & $\mathrm{H}$ & -11.46331 & -0.78413 & 0.50533 \\
\hline $\mathrm{C}$ & -8.27151 & 1.36215 & 1.26641 & $\mathrm{H}$ & -7.41960 & 1.75184 & -1.43901 \\
\hline $\mathrm{C}$ & 1.17463 & 1.07737 & -0.20011 & $\mathrm{H}$ & -9.16186 & 1.85659 & -1.18823 \\
\hline $\mathrm{C}$ & 1.26672 & 3.59731 & -0.39620 & $\mathrm{H}$ & -8.49161 & 0.48331 & -2.06523 \\
\hline $\mathrm{C}$ & -7.23357 & 1.68789 & 2.04144 & $\mathrm{H}$ & -9.33821 & -1.28573 & -0.66193 \\
\hline $\mathrm{C}$ & -3.89753 & -0.04703 & -2.04413 & $\mathrm{H}$ & -9.11777 & -1.29034 & 1.08666 \\
\hline $\mathrm{C}$ & -1.93492 & -0.51053 & 0.27160 & $\mathrm{H}$ & 5.15762 & 5.41509 & 0.77417 \\
\hline $\mathrm{C}$ & -8.16722 & 0.38220 & 0.09745 & $\mathrm{H}$ & 4.98113 & 6.03276 & -0.88716 \\
\hline $\mathrm{C}$ & 4.21817 & 3.02269 & 0.18443 & $\mathrm{H}$ & -0.47703 & 3.65039 & 0.87575 \\
\hline $\mathrm{C}$ & 6.72170 & 3.25050 & 0.16842 & $\mathrm{H}$ & -0.70808 & 4.37467 & -0.73687 \\
\hline $\mathrm{C}$ & -10.69965 & -0.00167 & 0.41181 & $\mathrm{H}$ & 6.77006 & -2.42524 & -0.41707 \\
\hline $\mathrm{C}$ & -8.31780 & 1.16273 & -1.22453 & $\mathrm{H}$ & -10.49351 & 0.26497 & 2.53418 \\
\hline $\mathrm{C}$ & -9.32365 & -0.64153 & 0.22371 & $\mathrm{H}$ & -9.93889 & 2.62151 & 0.70471 \\
\hline $\mathrm{C}$ & 5.17876 & 5.14137 & -0.29065 & $\mathrm{H}$ & -9.61520 & 2.56784 & 2.43813 \\
\hline $\mathrm{C}$ & -0.23997 & 3.55166 & -0.19438 & $\mathrm{H}$ & 6.53990 & 4.27830 & -1.71083 \\
\hline $\mathrm{C}$ & 6.43205 & -2.34882 & 0.61152 & $\mathrm{H}$ & -3.31005 & -3.00224 & -3.06478 \\
\hline $\mathrm{C}$ & -10.71283 & 0.88596 & 1.64961 & $\mathrm{H}$ & -2.79410 & -4.06788 & -1.74077 \\
\hline $\mathrm{C}$ & 6.08796 & -1.09105 & 1.12499 & $\mathrm{H}$ & -1.73792 & -3.79496 & -3.14227 \\
\hline $\mathrm{C}$ & 6.18977 & 0.08139 & 0.23105 & $\mathrm{H}$ & 6.57337 & -4.46202 & 1.01949 \\
\hline $\mathrm{C}$ & -9.63697 & 1.95872 & 1.5 & $\mathrm{H}$ & 0.24364 & -2.52909 & -3.32255 \\
\hline $\mathrm{C}$ & 6.53150 & 4.53151 & -0.63858 & $\mathrm{H}$ & 1.00278 & -1.36011 & -2.26016 \\
\hline $\mathrm{C}$ & -2.45600 & -3.32222 & -2.46439 & $\mathrm{H}$ & 5.39839 & -0.00933 & 2.85114 \\
\hline $\mathrm{C}$ & 6.32628 & -3.47721 & 1.40177 & $\mathrm{H}$ & 5.24544 & -1.99755 & 4.28736 \\
\hline $\mathrm{C}$ & 0.67265 & -2.40932 & -2.32109 & $\mathrm{H}$ & 1.52155 & -4.38731 & -2.19100 \\
\hline $\mathrm{C}$ & 5.66279 & -0.98131 & 2.44840 & $\mathrm{H}$ & 2.18027 & -3.23046 & -1.04476 \\
\hline $\mathrm{C}$ & 5.89689 & -3.36228 & 2.73443 & $\mathrm{H}$ & 3.23208 & -2.00513 & -3.01334 \\
\hline $\mathrm{C}$ & 5.57354 & -2.10686 & 3.26105 & $\mathrm{H}$ & 2.55725 & -4.61222 & -4.47895 \\
\hline $\mathrm{C}$ & 1.85265 & -3.34423 & -2.08221 & $\mathrm{H}$ & 3.59833 & -3.32249 & -5.10432 \\
\hline $\mathrm{C}$ & 3.04039 & -3.08597 & -3.01449 & $\mathrm{H}$ & 1.87236 & -3.02454 & -4.86630 \\
\hline $\mathrm{C}$ & 2.74748 & -3.53222 & -4.44766 & $\mathrm{H}$ & 5.15281 & -3.61592 & -3.1207 \\
\hline $\mathrm{C}$ & 4.28613 & -3.78544 & -2.47266 & $\mathrm{H}$ & 4.12339 & -4.86847 & -2.40474 \\
\hline $\mathrm{C}$ & 5.38848 & -4.45783 & 4.77651 & $\mathrm{H}$ & 4.52926 & -3.41405 & -1.47052 \\
\hline $\mathrm{H}$ & -2.01348 & -3.07499 & 0.57414 & $\mathrm{H}$ & 5.40178 & -5.48390 & 5.1408 \\
\hline $\mathrm{H}$ & 2.49329 & 3.72856 & -1.86295 & $\mathrm{H}$ & 4.37061 & -4.05863 & 4.83786 \\
\hline $\mathrm{H}$ & 7.84798 & 2.02089 & -0.84724 & $\mathrm{H}$ & 6.06102 & -3.84332 & 5.3837 \\
\hline
\end{tabular}


Structure, energy and Cartesian coordinate of 1-Bf:

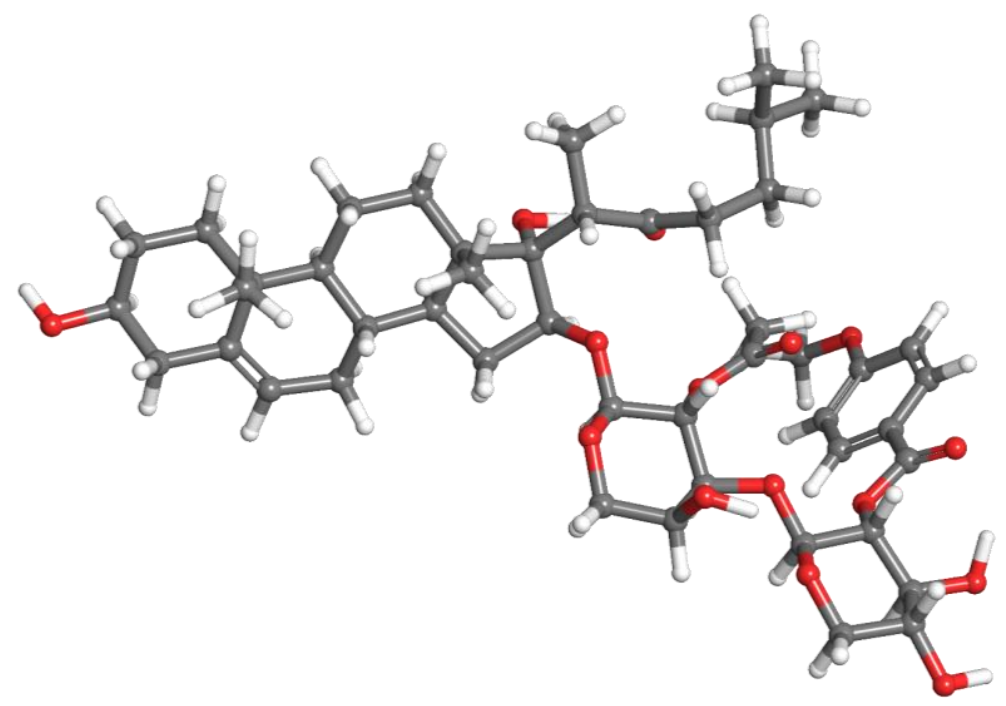

1-Bf $(\Delta G=1.5 \mathrm{kcal} / \mathrm{mol}$, Boltzmann pop. $(298 \mathrm{~K})=2.5 \%)$

M06-2X/6-311+G(d,p)-PCM(MeOH)//M06-2X/6-31G(d)-PCM(MeOH):

Gibbs Free Energy (a.u.) $=-2958.9922708$

M06-2X/6-311+G(d,p)-PCM(MeOH): $\quad$ Electronic energy (a.u. $)=-2960.0314998$

M06-2X/6-31G(d)-PCM(MeOH): Zero-point correction $($ a.u. $)=1.135122$

Thermal correction to Energy (a.u.) $=1.195292$

Thermal correction to Enthalpy (a.u.) $=1.196236$

Thermal correction to Gibbs Free Energy (a.u.) $=1.039229$

$\begin{array}{lrrrrrrr}\mathrm{O} & 0.83615 & -0.18254 & -0.82900 & \mathrm{H} & -8.68319 & -4.75968 & -0.65104 \\ \mathrm{O} & -3.48554 & -2.02140 & -0.60336 & \mathrm{H} & 12.31338 & -1.40452 & 2.36195 \\ \mathrm{O} & -5.71542 & -1.21165 & 0.79453 & \mathrm{H} & -2.64173 & 1.57917 & 1.98646 \\ \mathrm{O} & -1.68985 & -0.00514 & 0.33868 & \mathrm{H} & -3.68762 & 2.50127 & 0.86011 \\ \mathrm{O} & 2.77320 & 2.55011 & 0.36101 & \mathrm{H} & -1.91630 & 2.72951 & 0.84346 \\ \mathrm{O} & 0.55793 & -2.39708 & -1.15661 & \mathrm{H} & -2.10620 & -2.47099 & 0.87244 \\ \mathrm{O} & -1.85386 & -3.43419 & -2.33114 & \mathrm{H} & 2.61415 & -0.96701 & 1.64412 \\ \mathrm{O} & -4.49186 & -3.95462 & -1.22545 & \mathrm{H} & 2.76451 & -1.56459 & -0.01464 \\ \mathrm{O} & -8.20342 & -2.53939 & -0.00728 & \mathrm{H} & 5.23984 & -0.80969 & 2.66256 \\ \mathrm{O} & -7.85178 & -5.24674 & -0.77663 & \mathrm{H} & 4.78054 & -2.32086 & 1.90352 \\ \mathrm{O} & 11.61395 & -2.05407 & 2.19593 & \mathrm{H} & 4.22482 & 0.79403 & 1.25410 \\ \mathrm{O} & 0.13911 & 2.90412 & -0.36402 & \mathrm{H} & 6.68207 & 0.77099 & 1.19948 \\ \mathrm{O} & -3.08994 & 0.94726 & -1.15257 & \mathrm{H} & -5.89179 & -1.69534 & -1.20625 \\ \mathrm{O} & -7.12955 & 0.10003 & -0.36437 & \mathrm{H} & 1.74874 & 1.27800 & -2.58322 \\ \mathrm{O} & -5.33280 & 3.99755 & 4.30696 & \mathrm{H} & 5.13572 & 2.52007 & -0.10047 \\ \mathrm{C} & -2.72419 & 2.00418 & 0.98584 & \mathrm{H} & 5.14136 & 2.42402 & -1.86132 \\ \mathrm{C} & -2.14961 & -2.30754 & -0.21529 & \mathrm{H} & 7.32671 & 1.75030 & -0.95140 \\ \mathrm{C} & 2.80800 & -0.66450 & 0.61114 & \mathrm{H} & 6.56694 & 0.44313 & -1.83568 \\ \mathrm{C} & 2.58531 & 1.51514 & -0.59423 & \mathrm{H} & 0.35335 & -1.49158 & 0.68522\end{array}$




\begin{tabular}{|c|c|c|c|c|c|c|c|}
\hline $\mathrm{C}$ & 5.51628 & -1.50756 & 1.85564 & $\mathrm{H}$ & 5.39550 & -1.55237 & -0.28115 \\
\hline $\mathrm{C}$ & 4.14862 & 0.05184 & 0.44494 & $\mathrm{H}$ & -1.55338 & -0.76751 & -1.59531 \\
\hline $\mathrm{C}$ & 0.43337 & 2.50215 & -1.48137 & $\mathrm{H}$ & -2.03106 & -4.44445 & -0.52709 \\
\hline $\mathrm{C}$ & 6.64841 & 0.12028 & 0.30856 & $\mathrm{H}$ & 6.98355 & -2.78352 & 2.91875 \\
\hline $\mathrm{C}$ & 3.97850 & 0.86964 & -0.85239 & $\mathrm{H}$ & 4.74589 & -0.71349 & -2.16022 \\
\hline $\mathrm{C}$ & -5.78698 & -2.19322 & -0.23607 & $\mathrm{H}$ & 3.89633 & 0.57513 & -3.00830 \\
\hline $\mathrm{C}$ & 1.84580 & 2.06544 & -1.83080 & $\mathrm{H}$ & 2.98268 & -0.63824 & -2.09928 \\
\hline $\mathrm{C}$ & 5.17430 & 1.82380 & -0.94564 & $\mathrm{H}$ & 1.20741 & 0.80439 & 0.93027 \\
\hline $\mathrm{C}$ & 6.49462 & 1.03814 & -0.91840 & $\mathrm{H}$ & -4.24536 & -3.38027 & 0.74769 \\
\hline $\mathrm{C}$ & 0.14457 & -1.29823 & -0.38126 & $\mathrm{H}$ & -6.81402 & -3.61993 & 0.99126 \\
\hline $\mathrm{C}$ & 5.41872 & -0.78852 & 0.50981 & $\mathrm{H}$ & 10.81160 & -0.96567 & -0.08250 \\
\hline $\mathrm{C}$ & -2.56018 & 0.94447 & -0.06692 & $\mathrm{H}$ & 11.27290 & 0.36459 & 0.98135 \\
\hline $\mathrm{C}$ & 7.98285 & -1.72224 & 1.42296 & $\mathrm{H}$ & 7.29497 & -1.94866 & -1.34760 \\
\hline $\mathrm{C}$ & -1.35090 & -1.05946 & -0.56172 & $\mathrm{H}$ & 9.01189 & -2.12550 & -0.99102 \\
\hline $\mathrm{C}$ & -1.58640 & -3.53259 & -0.95099 & $\mathrm{H}$ & 8.44425 & -0.68754 & -1.83783 \\
\hline $\mathrm{C}$ & 6.88893 & -2.05529 & 2.11311 & $\mathrm{H}$ & 9.24444 & 0.98559 & -0.29398 \\
\hline $\mathrm{C}$ & 3.89417 & -0.03202 & -2.09555 & $\mathrm{H}$ & 8.90871 & 0.90943 & 1.43440 \\
\hline $\mathrm{C}$ & 1.77455 & 0.36456 & 0.10097 & $\mathrm{H}$ & -5.35297 & -5.40511 & -0.00412 \\
\hline $\mathrm{C}$ & 7.98070 & -0.68255 & 0.30168 & $\mathrm{H}$ & -5.39105 & -5.69191 & -1.76180 \\
\hline $\mathrm{C}$ & -4.45434 & -2.93560 & -0.24090 & $\mathrm{H}$ & 0.14959 & -3.81855 & 0.29920 \\
\hline $\mathrm{C}$ & -6.93439 & -3.15982 & 0.00018 & $\mathrm{H}$ & 0.33613 & -4.39334 & -1.37847 \\
\hline $\mathrm{C}$ & 10.49748 & -0.38981 & 0.79773 & $\mathrm{H}$ & -4.93470 & -0.32244 & 2.88882 \\
\hline $\mathrm{C}$ & 8.19470 & -1.40139 & -1.04626 & $\mathrm{H}$ & 10.14473 & -0.75821 & 2.88533 \\
\hline $\mathrm{C}$ & 9.15357 & 0.29845 & 0.55418 & $\mathrm{H}$ & 9.64857 & -3.00149 & 0.90426 \\
\hline $\mathrm{C}$ & -5.49855 & -4.93283 & -0.98637 & $\mathrm{H}$ & 9.21479 & -3.02398 & 2.61395 \\
\hline $\mathrm{C}$ & -0.07885 & -3.59995 & -0.75463 & $\mathrm{H}$ & -7.01078 & -3.82861 & -2.04112 \\
\hline $\mathrm{C}$ & -5.32910 & 0.67697 & 2.73982 & $\mathrm{H}$ & 3.43179 & 2.97019 & -3.02371 \\
\hline $\mathrm{C}$ & 10.40510 & -1.33959 & 1.98526 & $\mathrm{H}$ & 2.85542 & 3.98104 & -1.68085 \\
\hline $\mathrm{C}$ & -6.09436 & 0.95856 & 1.60784 & $\mathrm{H}$ & 1.88507 & 3.80507 & -3.15755 \\
\hline $\mathrm{C}$ & -6.38381 & -0.06332 & 0.58220 & $\mathrm{H}$ & -4.46586 & 1.42434 & 4.55135 \\
\hline $\mathrm{C}$ & 9.31171 & -2.37136 & 1.73998 & $\mathrm{H}$ & -0.08170 & 2.51123 & -3.56187 \\
\hline $\mathrm{C}$ & -6.87064 & -4.27283 & -1.04295 & $\mathrm{H}$ & -1.03376 & 1.45332 & -2.53957 \\
\hline $\mathrm{C}$ & 2.54869 & 3.28016 & -2.46123 & $\mathrm{H}$ & -7.19468 & 2.46508 & 0.53692 \\
\hline $\mathrm{C}$ & -5.05928 & 1.66679 & 3.67839 & $\mathrm{H}$ & -6.69296 & 4.25942 & 2.20185 \\
\hline $\mathrm{C}$ & -0.59218 & 2.45852 & -2.59400 & $\mathrm{H}$ & -2.11488 & 3.43754 & -1.45288 \\
\hline $\mathrm{C}$ & -6.59786 & 2.25323 & 1.41803 & $\mathrm{H}$ & -2.48486 & 3.31045 & -3.16629 \\
\hline $\mathrm{C}$ & -5.54890 & 2.96157 & 3.47159 & $\mathrm{H}$ & -0.28036 & 5.10239 & -2.04863 \\
\hline $\mathrm{C}$ & -6.32243 & 3.24866 & 2.33461 & $\mathrm{H}$ & -3.15763 & 5.82114 & -2.80586 \\
\hline $\mathrm{C}$ & -1.67762 & 3.52199 & -2.45322 & $\mathrm{H}$ & -1.89719 & 6.98859 & -2.37397 \\
\hline $\mathrm{C}$ & -1.17437 & 4.95440 & -2.67338 & $\mathrm{H}$ & -2.48324 & 5.82745 & -1.16701 \\
\hline $\mathrm{C}$ & -2.23660 & 5.95823 & -2.22580 & $\mathrm{H}$ & -0.41615 & 6.22375 & -4.26970 \\
\hline $\mathrm{C}$ & -0.79529 & 5.20574 & -4.13395 & $\mathrm{H}$ & -1.67562 & 5.08550 & -4.77744 \\
\hline $\mathrm{C}$ & -4.52867 & 3.77026 & 5.45492 & $\mathrm{H}$ & -0.02523 & 4.51501 & -4.49273 \\
\hline $\mathrm{H}$ & 1.88138 & 2.90876 & 0.52540 & $\mathrm{H}$ & -4.46563 & 4.72771 & 5.96989 \\
\hline $\mathrm{H}$ & -2.82267 & -3.46602 & -2.41455 & $\mathrm{H}$ & -3.52481 & 3.43892 & 5.16939 \\
\hline $\mathrm{H}$ & -8.14628 & -1.72840 & -0.54196 & $\mathrm{H}$ & -4.98744 & 3.02712 & 6.11516 \\
\hline
\end{tabular}


Structure, energy and Cartesian coordinate of 1-Bg:

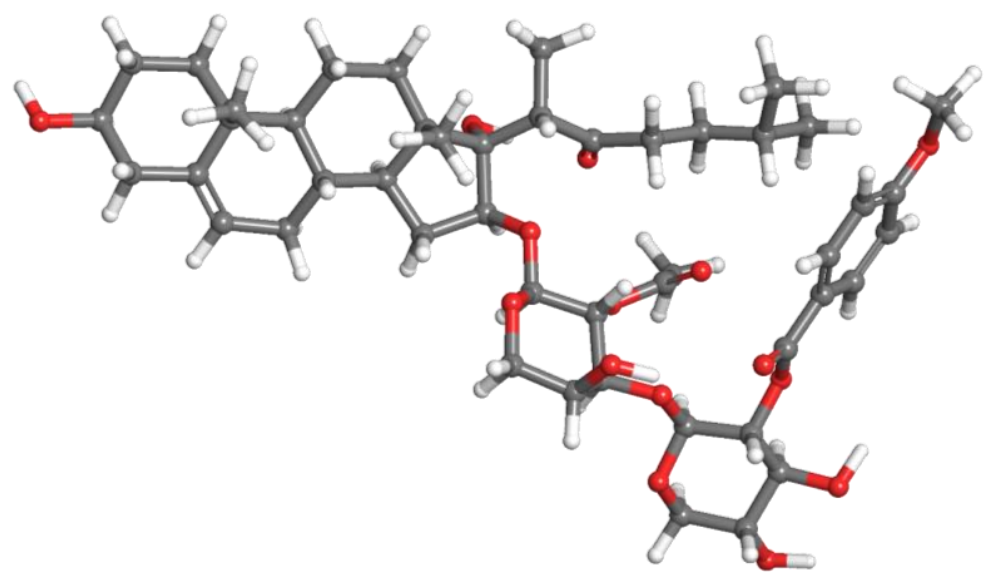

1-Bg $(\Delta G=2.4 \mathrm{kcal} / \mathrm{mol}$, Boltzmann pop. $(298 \mathrm{~K})=0.6 \%)$

M06-2X/6-311+G(d,p)-PCM(MeOH)//M06-2X/6-31G(d)-PCM(MeOH):

Gibbs Free Energy (a.u.) = -2958.9908446

M06-2X/6-311+G(d,p)-PCM(MeOH): $\quad$ Electronic energy (a.u. $)=-2960.0317096$

M06-2X/6-31G(d)-PCM(MeOH): Zero-point correction (a.u.) = 1.134646

Thermal correction to Energy (a.u.) $=1.194723$

Thermal correction to Enthalpy (a.u.) $=1.195668$

Thermal correction to Gibbs Free Energy (a.u.) $=1.040865$

$\begin{array}{lrrr}\mathrm{O} & -0.88844 & -0.10031 & 0.03853 \\ \mathrm{O} & 3.10790 & -2.57707 & 0.76985 \\ \mathrm{O} & 5.58664 & -1.35833 & 0.13070 \\ \mathrm{O} & 1.44508 & -1.07580 & -1.15516 \\ \mathrm{O} & -2.88747 & 1.71298 & -2.27411 \\ \mathrm{O} & -0.78170 & -1.69266 & 1.63107 \\ \mathrm{O} & 1.70619 & -2.21674 & 2.96761 \\ \mathrm{O} & 3.75817 & -4.49711 & -0.25671 \\ \mathrm{O} & 7.66794 & -3.26329 & -0.41632 \\ \mathrm{O} & 6.81533 & -5.47247 & -1.94501 \\ \mathrm{O} & -12.16597 & -1.37955 & 0.30005 \\ \mathrm{O} & -0.12981 & 1.88403 & -2.29562 \\ \mathrm{O} & 3.10165 & 0.33343 & -0.55509 \\ \mathrm{O} & 4.95649 & -1.11825 & 2.28890 \\ \mathrm{O} & 6.92945 & 4.73551 & 0.72246 \\ \mathrm{C} & 2.62717 & -0.03491 & -2.89542 \\ \mathrm{C} & 1.70169 & -2.69191 & 0.61658 \\ \mathrm{C} & -3.15174 & -0.97628 & -0.53482 \\ \mathrm{C} & -2.54069 & 1.41569 & -0.92819 \\ \mathrm{C} & -6.11611 & -1.87568 & -0.61603 \\ \mathrm{C} & -4.37697 & -0.06625 & -0.63441\end{array}$

$\begin{array}{lrrr}\mathrm{H} & 7.72855 & -5.18176 & -1.78846 \\ \mathrm{H} & -12.84060 & -0.81511 & -0.10604 \\ \mathrm{H} & 1.76302 & 0.51540 & -3.28001 \\ \mathrm{H} & 2.66428 & -1.00775 & -3.39136 \\ \mathrm{H} & 3.54120 & 0.52659 & -3.08689 \\ \mathrm{H} & 1.45236 & -3.45588 & -0.13019 \\ \mathrm{H} & -3.21063 & -1.84187 & -1.20074 \\ \mathrm{H} & -3.02569 & -1.35029 & 0.48843 \\ \mathrm{H} & -5.97651 & -1.83401 & -1.70841 \\ \mathrm{H} & -5.46518 & -2.68762 & -0.26659 \\ \mathrm{H} & -4.59149 & 0.07835 & -1.70392 \\ \mathrm{H} & -6.96077 & 0.54023 & -1.29133 \\ \mathrm{H} & 5.53052 & -3.13874 & 1.18887 \\ \mathrm{H} & -1.29009 & 2.23926 & 0.64228 \\ \mathrm{H} & -5.07245 & 2.40806 & -1.52475 \\ \mathrm{H} & -4.68546 & 3.32876 & -0.07087 \\ \mathrm{H} & -7.05350 & 2.66332 & -0.06756 \\ \mathrm{H} & -6.19198 & 1.97426 & 1.29256 \\ \mathrm{H} & -0.80870 & -2.11164 & -0.39205 \\ \mathrm{H} & -5.52190 & -0.70891 & 1.07858 \\ \mathrm{H} & 1.51571 & -0.55855 & 0.85674\end{array}$




\begin{tabular}{|c|c|c|c|c|c|c|c|}
\hline $\mathrm{C}$ & -0.19025 & 2.31859 & -1.15376 & $\mathrm{H}$ & 1.46763 & -4.13312 & 2.19258 \\
\hline $\mathrm{C}$ & -6.77191 & 0.51758 & -0.20402 & $\mathrm{H}$ & -7.86219 & -3.23427 & -0.50203 \\
\hline $\mathrm{C}$ & -3.86982 & 1.30043 & -0.12598 & $\mathrm{H}$ & -4.40234 & 0.91933 & 1.96600 \\
\hline $\mathrm{C}$ & 5.35828 & -2.76334 & 0.17541 & $\mathrm{H}$ & -3.31082 & 2.28252 & 1.73500 \\
\hline $\mathrm{C}$ & -1.51108 & 2.44040 & -0.40978 & $\mathrm{H}$ & -2.69863 & 0.62589 & 1.60507 \\
\hline $\mathrm{C}$ & -4.95847 & 2.33344 & -0.43746 & $\mathrm{H}$ & -1.56772 & -0.27684 & -1.88725 \\
\hline $\mathrm{C}$ & -6.29349 & 1.92188 & 0.20294 & $\mathrm{H}$ & 3.67914 & -2.67287 & -1.20956 \\
\hline $\mathrm{C}$ & -0.40069 & -1.36681 & 0.31344 & $\mathrm{H}$ & 6.16101 & -2.95095 & -1.80595 \\
\hline $\mathrm{C}$ & -5.67609 & -0.54732 & 0.00170 & $\mathrm{H}$ & -10.79459 & 0.62827 & 1.35591 \\
\hline $\mathrm{C}$ & 2.45035 & -0.21223 & -1.41442 & $\mathrm{H}$ & -11.40218 & 1.18928 & -0.20303 \\
\hline $\mathrm{C}$ & -8.43855 & -1.35010 & 0.19191 & $\mathrm{H}$ & -7.16379 & -0.09819 & 2.42607 \\
\hline $\mathrm{C}$ & 1.12101 & -1.34575 & 0.20974 & $\mathrm{H}$ & -8.92525 & -0.11952 & 2.49390 \\
\hline $\mathrm{C}$ & 1.17376 & -3.09182 & 1.99426 & $\mathrm{H}$ & -8.08382 & 1.40784 & 2.23871 \\
\hline $\mathrm{C}$ & -7.54597 & -2.20938 & -0.30670 & $\mathrm{H}$ & -9.10136 & 2.03139 & 0.13806 \\
\hline $\mathrm{C}$ & -3.55151 & 1.27394 & 1.37871 & $\mathrm{H}$ & -9.17466 & 0.93089 & -1.23665 \\
\hline $\mathrm{C}$ & -1.97118 & -0.04580 & -0.89400 & $\mathrm{H}$ & 4.34746 & -4.67801 & -2.24676 \\
\hline $\mathrm{C}$ & -8.11720 & 0.11908 & 0.46762 & $\mathrm{H}$ & 4.31566 & -6.16530 & -1.26626 \\
\hline $\mathrm{C}$ & 3.92067 & -3.09671 & -0.21787 & $\mathrm{H}$ & -0.77822 & -3.75256 & 1.36693 \\
\hline $\mathrm{C}$ & 6.32063 & -3.40201 & -0.81368 & $\mathrm{H}$ & -0.70955 & -3.13865 & 3.03909 \\
\hline $\mathrm{C}$ & -10.65448 & 0.54051 & 0.27065 & $\mathrm{H}$ & 5.02009 & 1.28444 & 3.03462 \\
\hline $\mathrm{C}$ & -8.06919 & 0.34086 & 1.99354 & $\mathrm{H}$ & -10.81209 & -0.99319 & -1.22587 \\
\hline $\mathrm{C}$ & -9.25023 & 0.98274 & -0.14127 & $\mathrm{H}$ & -10.02585 & -1.78747 & 1.59489 \\
\hline $\mathrm{C}$ & 4.56535 & -5.10348 & -1.25550 & $\mathrm{H}$ & -9.98973 & -2.84111 & 0.18071 \\
\hline $\mathrm{C}$ & -0.34243 & -2.98425 & 2.02299 & $\mathrm{H}$ & 6.25185 & -5.35663 & 0.05248 \\
\hline $\mathrm{C}$ & 5.51187 & 1.65870 & 2.14246 & $\mathrm{H}$ & -2.74005 & 4.12992 & 0.20885 \\
\hline $\mathrm{C}$ & -10.89702 & -0.90910 & -0.12961 & $\mathrm{H}$ & -2.43262 & 4.04985 & -1.53871 \\
\hline $\mathrm{C}$ & 5.76296 & 0.77994 & 1.09042 & $\mathrm{H}$ & -1.16475 & 4.60195 & -0.42502 \\
\hline $\mathrm{C}$ & 5.37419 & -0.63974 & 1.25785 & $\mathrm{H}$ & 5.67307 & 3.66214 & 2.89066 \\
\hline $\mathrm{C}$ & -9.84536 & -1.80684 & 0.51036 & $\mathrm{H}$ & 0.75549 & 638 & 0.30125 \\
\hline $\mathrm{C}$ & 6.03794 & -4.89332 & -0.92314 & $\mathrm{H}$ & 1.43305 & 1.95434 & 0.13850 \\
\hline $\mathrm{C}$ & -1.99147 & 3.89540 & -0.55108 & $\mathrm{H}$ & 6.59559 & 0.57460 & -0.88857 \\
\hline $\mathrm{C}$ & 5.88471 & 2.99616 & 2.06262 & $\mathrm{H}$ & 7.28924 & 2.96047 & -1.03309 \\
\hline $\mathrm{C}$ & 1.04767 & 2.81071 & -0.43583 & $\mathrm{H}$ & 1.69830 & 4.20010 & -1.93659 \\
\hline $\mathrm{C}$ & 6.39940 & 1.25218 & -0.06494 & $\mathrm{H}$ & 2.30321 & 2.56545 & -2.15446 \\
\hline $\mathrm{C}$ & 6.52850 & 3.45615 & 0.90979 & $\mathrm{H}$ & 3.87198 & 2.84663 & -0.27787 \\
\hline $\mathrm{C}$ & 6.78633 & 2.57612 & -0.15181 & $\mathrm{H}$ & 2.71264 & 5.68057 & -0.08864 \\
\hline $\mathrm{C}$ & 2.11012 & 3.33327 & -1.39786 & $\mathrm{H}$ & 4.22394 & 5.16588 & 0.67859 \\
\hline $\mathrm{C}$ & 3.43494 & 3.73956 & -0.74802 & $\mathrm{H}$ & 2.69016 & 4.44709 & 1.18813 \\
\hline $\mathrm{C}$ & 3.24947 & 4.81540 & 0.32218 & $\mathrm{H}$ & 5.33972 & 4.57421 & -1.40130 \\
\hline $\mathrm{C}$ & 4.39086 & 4.23950 & -1.83256 & $\mathrm{H}$ & 3.94608 & 5.08937 & -2.36602 \\
\hline $\mathrm{C}$ & 6.84874 & 5.62539 & 1.82643 & $\mathrm{H}$ & 4.60461 & 3.45451 & -2.56712 \\
\hline $\mathrm{H}$ & -2.04022 & 1.72029 & -2.75570 & $\mathrm{H}$ & 7.28830 & 6.56263 & 1.48802 \\
\hline $\mathrm{H}$ & 2.65949 & -2.13606 & 2.78022 & $\mathrm{H}$ & 7.41372 & 5.23723 & 2.68035 \\
\hline $\mathrm{H}$ & 7.90907 & -2.32728 & -0.49088 & $\mathrm{H}$ & 5.80919 & 5.79791 & 2.1245 \\
\hline
\end{tabular}


Structure, energy and Cartesian coordinate of 1-Bh:

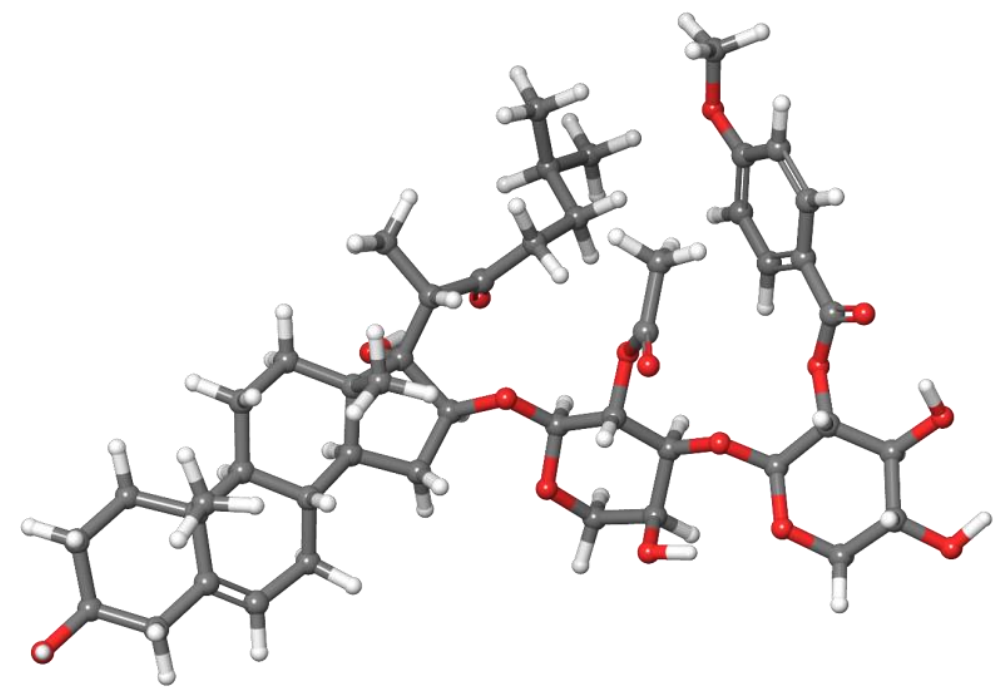

1-Bh $(\Delta G=2.5 \mathrm{kcal} / \mathrm{mol}$, Boltzmann pop. $(298 \mathrm{~K})=0.5 \%)$

M06-2X/6-311+G(d,p)-PCM(MeOH)//M06-2X/6-31G(d)-PCM(MeOH):

\begin{tabular}{rrr} 
Gibbs Free Energy (a.u.) & $=-2958.9907970$ \\
\hline M06-2X/6-311+G(d,p)-PCM(MeOH): $\quad$ Electronic energy (a.u.) & $=-2960.0314750$ \\
\hline M06-2X/6-31G(d)-PCM(MeOH): Zero-point correction $($ a.u. $)$ & $=1.134725$ \\
Thermal correction to Energy (a.u. $)$ & $=1.194846$ \\
Thermal correction to Enthalpy (a.u.) & $=1.195791$ \\
Thermal correction to Gibbs Free Energy (a.u. $)$ & $=1.040678$
\end{tabular}

$\begin{array}{lrrrrrrr}\mathrm{O} & 0.79555 & 0.08547 & 0.34012 & \mathrm{H} & -7.97265 & -5.82434 & -0.20601 \\ \mathrm{O} & -3.19955 & -2.41230 & -0.04165 & \mathrm{H} & 11.87414 & -2.46560 & -1.42917 \\ \mathrm{O} & -5.78092 & -1.48160 & -0.06050 & \mathrm{H} & -3.50266 & 1.74907 & -1.23102 \\ \mathrm{O} & -1.95209 & 0.03239 & -0.37847 & \mathrm{H} & -3.76994 & 1.02189 & -2.84662 \\ \mathrm{O} & 3.15880 & 2.38685 & 1.76696 & \mathrm{H} & -2.30139 & 1.95866 & -2.52738 \\ \mathrm{O} & 0.49164 & -1.80179 & 1.58025 & \mathrm{H} & -2.75841 & -1.33097 & 1.66093 \\ \mathrm{O} & -1.04502 & -4.04037 & 0.66183 & \mathrm{H} & 3.12511 & -1.31951 & 1.86136 \\ \mathrm{O} & -3.89438 & -4.53214 & 0.30738 & \mathrm{H} & 2.78086 & -1.36357 & 0.13363 \\ \mathrm{O} & -7.79047 & -3.55912 & -0.79699 & \mathrm{H} & 5.94380 & -1.42564 & 2.07761 \\ \mathrm{O} & -7.16478 & -6.10113 & 0.25756 & \mathrm{H} & 5.18265 & -2.62404 & 1.05040 \\ \mathrm{O} & 11.89806 & -2.24304 & -0.48534 & \mathrm{H} & 4.68923 & 0.51673 & 1.59175 \\ \mathrm{O} & 0.45779 & 2.86952 & 1.98303 & \mathrm{H} & 7.02215 & 0.57468 & 0.84173 \\ \mathrm{O} & -2.02525 & -0.89311 & -2.43706 & \mathrm{H} & -5.22652 & -2.92591 & -1.43458 \\ \mathrm{O} & -6.76293 & -1.18494 & -2.06027 & \mathrm{H} & 1.28581 & 2.16858 & -1.01932 \\ \mathrm{O} & -5.56059 & 4.74601 & -0.01475 & \mathrm{H} & 5.29424 & 2.60649 & 0.65322 \\ \mathrm{C} & -3.02648 & 1.26642 & -2.08656 & \mathrm{H} & 4.80583 & 3.07771 & -0.97469 \\ \mathrm{C} & -2.25123 & -1.96066 & 0.91319 & \mathrm{H} & 7.10773 & 2.20841 & -0.98238 \\ \mathrm{C} & 3.05640 & -0.70218 & 0.96281 & \mathrm{H} & 6.04800 & 1.23212 & -1.97746 \\ \mathrm{C} & 2.64329 & 1.73351 & 0.61338 & \mathrm{H} & -0.52993 & -0.03980 & 1.90243 \\ \mathrm{C} & 5.93304 & -1.82292 & 1.04976 & \mathrm{H} & 5.22514 & -1.18617 & -0.86648\end{array}$




\begin{tabular}{|c|c|c|c|c|c|c|c|}
\hline $\mathrm{C}$ & 4.33767 & 0.06773 & 0.65069 & $\mathrm{H}$ & -0.82387 & -1.68105 & -0.67999 \\
\hline $\mathrm{C}$ & 0.40595 & 2.88339 & 0.76130 & $\mathrm{H}$ & -2.23261 & -3.61363 & 2.31024 \\
\hline $\mathrm{C}$ & 6.70059 & 0.24236 & -0.16058 & $\mathrm{H}$ & 7.54202 & -3.34160 & 1.18673 \\
\hline $\mathrm{C}$ & 3.86659 & 1.25240 & -0.21899 & $\mathrm{H}$ & 4.12537 & 0.18882 & -2.11993 \\
\hline $\mathrm{C}$ & -5.48398 & -2.84122 & -0.37247 & $\mathrm{H}$ & 3.17950 & 1.67158 & -2.24010 \\
\hline $\mathrm{C}$ & 1.63663 & 2.65341 & -0.10326 & $\mathrm{H}$ & 2.45738 & 0.21416 & -1.54367 \\
\hline $\mathrm{C}$ & 5.05078 & 2.21775 & -0.34197 & $\mathrm{H}$ & 1.67738 & 0.53553 & 2.14258 \\
\hline $\mathrm{C}$ & 6.27244 & 1.50023 & -0.93903 & $\mathrm{H}$ & -4.43987 & -2.94509 & 1.51718 \\
\hline $\mathrm{C}$ & -0.13465 & -0.64758 & 1.06458 & $\mathrm{H}$ & -6.90282 & -3.60570 & 1.03594 \\
\hline $\mathrm{C}$ & 5.51670 & -0.71451 & 0.08378 & $\mathrm{H}$ & 10.51563 & -0.52343 & -2.03021 \\
\hline $\mathrm{C}$ & -2.30164 & 0.00932 & -1.68729 & $\mathrm{H}$ & 11.34503 & 0.36699 & -0.75336 \\
\hline $\mathrm{C}$ & 8.15862 & -1.82968 & -0.11658 & $\mathrm{H}$ & 6.73296 & -1.14870 & -2.50672 \\
\hline $\mathrm{C}$ & -1.25912 & -1.10197 & 0.13702 & $\mathrm{H}$ & 8.46701 & -1.38403 & -2.72216 \\
\hline $\mathrm{C}$ & -1.53676 & -3.12393 & 1.61209 & $\mathrm{H}$ & 7.78605 & 0.23571 & -2.85961 \\
\hline $\mathrm{C}$ & 7.27963 & -2.39376 & 0.71632 & $\mathrm{H}$ & 9.08676 & 1.32568 & -1.13705 \\
\hline $\mathrm{C}$ & 3.37995 & 0.80018 & -1.60549 & $\mathrm{H}$ & 9.23185 & 0.66411 & 0.48981 \\
\hline $\mathrm{C}$ & 1.97701 & 0.39246 & 1.09793 & $\mathrm{H}$ & -5.18485 & -5.26411 & 1.77050 \\
\hline $\mathrm{C}$ & 7.92350 & -0.48061 & -0.79621 & $\mathrm{H}$ & -4.53035 & -6.43572 & 0.59811 \\
\hline $\mathrm{C}$ & -4.24488 & -3.17033 & 0.45439 & $\mathrm{H}$ & -0.73469 & -1.96285 & 3.24678 \\
\hline $\mathrm{C}$ & -6.63120 & -3.76825 & -0.01801 & $\mathrm{H}$ & 0.23765 & -3.39048 & 2.80028 \\
\hline $\mathrm{C}$ & 10.49696 & -0.29640 & -0.95440 & $\mathrm{H}$ & -5.33453 & 0.46331 & 1.23490 \\
\hline $\mathrm{C}$ & 7.71544 & -0.70704 & -2.30754 & $\mathrm{H}$ & 10.73267 & -1.36217 & 0.89408 \\
\hline $\mathrm{C}$ & 9.18483 & 0.39126 & -0.57378 & $\mathrm{H}$ & 9.50937 & -2.83112 & -1.46943 \\
\hline $\mathrm{C}$ & -4.92639 & -5.42644 & 0.71395 & $\mathrm{H}$ & 9.57670 & -3.42011 & 0.19195 \\
\hline $\mathrm{C}$ & -0.36515 & -2.57013 & 2.40747 & $\mathrm{H}$ & -5.87955 & -5.39000 & -1.21134 \\
\hline $\mathrm{C}$ & -5.63058 & 1.17637 & 0.47363 & $\mathrm{H}$ & 2.88772 & 3.95417 & -1.32526 \\
\hline $\mathrm{C}$ & 10.67285 & -1.59463 & -0.17696 & $\mathrm{H}$ & 2.79714 & 4.42138 & 0.38622 \\
\hline $\mathrm{C}$ & -6.17618 & 0.72275 & -0.73698 & $\mathrm{H}$ & 1.43862 & 4.74648 & -0.70978 \\
\hline $\mathrm{C}$ & -6.29427 & -0.71744 & -1.03941 & $\mathrm{H}$ & -5.04215 & 2.90380 & 1.62098 \\
\hline $\mathrm{C}$ & 9.47470 & -2.51460 & -0.41503 & $\mathrm{H}$ & -0.69313 & 3.77145 & -0.84092 \\
\hline $\mathrm{C}$ & -6.15589 & -5.21218 & -0.16009 & $\mathrm{H}$ & -1.12461 & 2.13614 & -0.40137 \\
\hline $\mathrm{C}$ & 2.22678 & 4.02849 & -0.45894 & $\mathrm{H}$ & -6.96274 & 1.28465 & -2.64976 \\
\hline $\mathrm{C}$ & -5.45985 & 2.52939 & 0.69189 & $\mathrm{H}$ & -6.63931 & 3.70672 & -2.29482 \\
\hline $\mathrm{C}$ & -0.88634 & 3.12056 & 0.02078 & $\mathrm{H}$ & -2.14345 & 2.93592 & 1.73948 \\
\hline $\mathrm{C}$ & -6.54637 & 1.64546 & -1.71450 & $\mathrm{H}$ & -2.96746 & 3.56208 & 0.31182 \\
\hline $\mathrm{C}$ & -5.80679 & 3.45292 & -0.30787 & $\mathrm{H}$ & -0.89521 & 5.13946 & 1.89078 \\
\hline $\mathrm{C}$ & -6.36022 & 3.00859 & -1.51515 & $\mathrm{H}$ & -3.95228 & 5.28173 & 1.96272 \\
\hline $\mathrm{C}$ & -2.03373 & 3.61939 & 0.88787 & $\mathrm{H}$ & -2.85861 & 6.37392 & 2.83220 \\
\hline $\mathrm{C}$ & -1.87525 & 5.05576 & 1.40164 & $\mathrm{H}$ & -2.92569 & 4.65676 & 3.27010 \\
\hline $\mathrm{C}$ & -2.96340 & 5.36028 & 2.43123 & $\mathrm{H}$ & -1.85472 & 7.09203 & 0.63695 \\
\hline $\mathrm{C}$ & -1.94432 & 6.06881 & 0.25767 & $\mathrm{H}$ & -2.91016 & 5.98214 & -0.25663 \\
\hline $\mathrm{C}$ & -5.88084 & 5.72701 & -0.99144 & $\mathrm{H}$ & -1.15182 & 5.92060 & -0.48310 \\
\hline $\mathrm{H}$ & 2.37403 & 2.64988 & 2.28231 & $\mathrm{H}$ & -5.59795 & 6.68406 & -0.55517 \\
\hline $\mathrm{H}$ & -1.82824 & -4.42226 & 0.22742 & $\mathrm{H}$ & -6.95309 & 5.72377 & -1.21142 \\
\hline $\mathrm{H}$ & -7.56290 & -2.98383 & -1.54910 & $\mathrm{H}$ & -5.31349 & 5.56007 & -1.91286 \\
\hline
\end{tabular}


Structure, energy and Cartesian coordinate of 1-Bi:

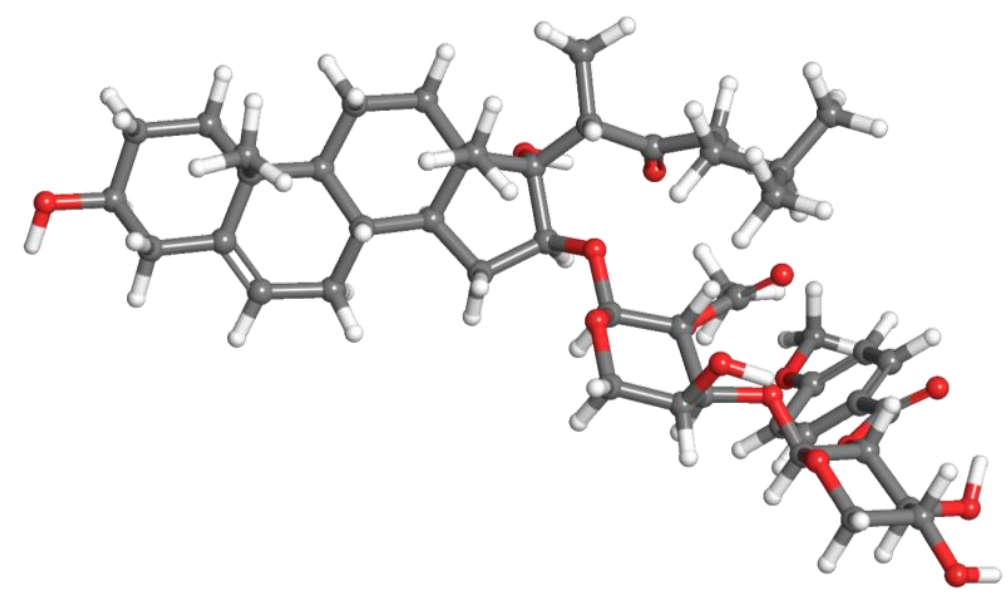

1-Bi $(\Delta G=2.6 \mathrm{kcal} / \mathrm{mol}$, Boltzmann pop. $(298 \mathrm{~K})=0.4 \%)$

M06-2X/6-311+G(d,p)-PCM(MeOH)//M06-2X/6-31G(d)-PCM(MeOH):

Gibbs Free Energy (a.u.) = -2958.9905521

M06-2X/6-311+G(d,p)-PCM(MeOH): $\quad$ Electronic energy (a.u. $)=-2960.0294561$

M06-2X/6-31G(d)-PCM(MeOH): Zero-point correction (a.u.) $=1.135249$

Thermal correction to Energy (a.u.) $=1.195577$

Thermal correction to Enthalpy (a.u.) $=1.196521$

Thermal correction to Gibbs Free Energy (a.u.) = 1.038904

$\begin{array}{lrrrrrrr}\mathrm{O} & -0.85248 & -0.59075 & 0.63071 & \mathrm{H} & 8.96809 & -3.33176 & -2.08665 \\ \mathrm{O} & 3.56283 & -1.55191 & -0.65535 & \mathrm{H} & -11.60748 & 0.24521 & -3.80057 \\ \mathrm{O} & 5.64987 & 0.11040 & -1.32931 & \mathrm{H} & 3.31244 & 3.04410 & 0.69037 \\ \mathrm{O} & 1.57033 & 0.48561 & -0.29357 & \mathrm{H} & 1.53793 & 3.01164 & 0.87578 \\ \mathrm{O} & -2.77070 & 2.25543 & 1.51818 & \mathrm{H} & 2.25670 & 2.82703 & -0.74080 \\ \mathrm{O} & -0.40076 & -2.53171 & -0.39815 & \mathrm{H} & 2.15305 & -1.20041 & -2.13042 \\ \mathrm{O} & 2.13113 & -3.83743 & -0.05628 & \mathrm{H} & -2.60888 & 0.41757 & -1.77356 \\ \mathrm{O} & 4.74545 & -3.39330 & -1.24076 & \mathrm{H} & -2.77668 & -1.10761 & -0.89031 \\ \mathrm{O} & 8.27080 & -1.19162 & -1.36755 & \mathrm{H} & -5.21054 & 1.27340 & -2.44254 \\ \mathrm{O} & 8.19076 & -3.88666 & -2.26648 & \mathrm{H} & -4.78353 & -0.37107 & -2.87121 \\ \mathrm{O} & -11.67368 & 0.20122 & -2.83497 & \mathrm{H} & -4.20484 & 1.52909 & -0.32692 \\ \mathrm{O} & -0.13655 & 1.98460 & 2.25797 & \mathrm{H} & -6.66662 & 1.51357 & -0.30500 \\ \mathrm{O} & 3.03057 & 0.55978 & 1.42600 & \mathrm{H} & 5.94599 & -1.39782 & 0.05030 \\ \mathrm{O} & 6.94722 & 0.66713 & 0.42291 & \mathrm{H} & -1.81075 & -0.65827 & 2.89490 \\ \mathrm{O} & 4.56685 & 6.26960 & -1.48958 & \mathrm{H} & -5.13625 & 1.94956 & 1.82305 \\ \mathrm{C} & 2.38641 & 2.60335 & 0.31828 & \mathrm{H} & -5.15869 & 0.71996 & 3.08703 \\ \mathrm{C} & 2.23511 & -1.67838 & -1.14236 & \mathrm{H} & -7.33720 & 0.82588 & 1.95081 \\ \mathrm{C} & -2.80968 & -0.01490 & -0.78929 & \mathrm{H} & -6.58816 & -0.74488 & 1.75026 \\ \mathrm{C} & -2.59431 & 0.84641 & 1.55167 & \mathrm{H} & -0.39501 & -0.71304 & -1.37433 \\ \mathrm{C} & -5.50945 & 0.22234 & -2.30033 & \mathrm{H} & -5.42014 & -1.22746 & -0.72877 \\ \mathrm{C} & -4.14873 & 0.43673 & -0.20423 & \mathrm{H} & 1.62329 & -1.23825 & 0.87004\end{array}$




\begin{tabular}{|c|c|c|c|c|c|c|c|}
\hline $\mathrm{C}$ & -0.42905 & 0.94696 & 2.83888 & $\mathrm{H}$ & 2.28056 & -3.61049 & -2.11227 \\
\hline $\mathrm{C}$ & -6.65060 & 0.43526 & -0.06924 & $\mathrm{H}$ & -6.97340 & 0.00360 & -3.94937 \\
\hline $\mathrm{C}$ & -3.99118 & 0.20043 & 1.31260 & $\mathrm{H}$ & -4.77218 & -1.84719 & 1.24308 \\
\hline $\mathrm{C}$ & 5.84677 & -1.26881 & -1.03315 & $\mathrm{H}$ & -3.94973 & -1.43657 & 2.74669 \\
\hline $\mathrm{C}$ & -1.86097 & 0.43096 & 2.84611 & $\mathrm{H}$ & -3.00866 & -1.76345 & 1.28406 \\
\hline $\mathrm{C}$ & -5.18513 & 0.86986 & 2.00235 & $\mathrm{H}$ & -1.20890 & 1.30648 & -0.06397 \\
\hline $\mathrm{C}$ & -6.50697 & 0.30812 & 1.45797 & $\mathrm{H}$ & 4.36581 & -1.82284 & -2.53492 \\
\hline $\mathrm{C}$ & -0.11727 & -1.15495 & -0.40190 & $\mathrm{H}$ & 6.95313 & -1.64875 & -2.83193 \\
\hline $\mathrm{C}$ & -5.42512 & -0.13115 & -0.81510 & $\mathrm{H}$ & -10.83205 & -0.56243 & -0.51455 \\
\hline $\mathrm{C}$ & 2.40045 & 1.12132 & 0.56152 & $\mathrm{H}$ & -11.26233 & 1.14593 & -0.43188 \\
\hline $\mathrm{C}$ & -7.98510 & -0.18250 & -2.13095 & $\mathrm{H}$ & -7.33470 & -2.20070 & -0.20136 \\
\hline $\mathrm{C}$ & 1.37014 & -0.92036 & -0.14568 & $\mathrm{H}$ & -9.04796 & -2.07396 & -0.59666 \\
\hline $\mathrm{C}$ & 1.79776 & -3.14775 & -1.23943 & $\mathrm{H}$ & -8.48397 & -1.56708 & 0.99344 \\
\hline $\mathrm{C}$ & -6.88417 & 0.00998 & -2.86288 & $\mathrm{H}$ & -9.24564 & 0.71914 & 0.94587 \\
\hline $\mathrm{C}$ & -3.92503 & -1.29664 & 1.65954 & $\mathrm{H}$ & -8.88414 & 1.80815 & -0.39097 \\
\hline $\mathrm{C}$ & -1.78027 & 0.43285 & 0.27124 & $\mathrm{H}$ & 5.68787 & -3.80464 & -3.05191 \\
\hline $\mathrm{C}$ & -7.98907 & -0.15016 & -0.60235 & $\mathrm{H}$ & 5.81537 & -5.03343 & -1.76827 \\
\hline $\mathrm{C}$ & 4.58277 & -2.00398 & -1.46787 & $\mathrm{H}$ & 0.02067 & -2.80382 & -2.41197 \\
\hline $\mathrm{C}$ & 7.06514 & -1.81961 & -1.75191 & $\mathrm{H}$ & -0.03769 & -4.25900 & -1.38280 \\
\hline $\mathrm{C}$ & -10.49731 & 0.44701 & -0.78727 & $\mathrm{H}$ & 6.74540 & 3.12095 & 1.00470 \\
\hline $\mathrm{C}$ & -8.22715 & -1.57904 & -0.07076 & $\mathrm{H}$ & -10.11969 & 1.55543 & -2.58318 \\
\hline $\mathrm{C}$ & -9.14869 & 0.77031 & -0.14396 & $\mathrm{H}$ & -9.67721 & -1.44975 & -2.59281 \\
\hline $\mathrm{C}$ & 5.82628 & -3.96219 & -1.97239 & $\mathrm{H}$ & -9.20948 & -0.34563 & -3.88852 \\
\hline $\mathrm{C}$ & 0.29040 & -3.21944 & -1.42948 & $\mathrm{H}$ & 7.26726 & -3.51306 & -0.44284 \\
\hline $\mathrm{C}$ & 6.15073 & 3.38508 & 0.13620 & $\mathrm{H}$ & -3.47287 & 0.40568 & 4.30990 \\
\hline $\mathrm{C}$ & -10.39962 & 0.52716 & -2.30035 & $\mathrm{H}$ & -2.77275 & 2.01208 & 4.02746 \\
\hline $\mathrm{C}$ & 5.77925 & 2.38332 & -0.76034 & $\mathrm{H}$ & -1.90083 & 0.80368 & 4.99735 \\
\hline $\mathrm{C}$ & 6.19979 & 0.99589 & -0.47757 & $\mathrm{H}$ & 6.05098 & 5.46291 & 0.64989 \\
\hline $\mathrm{C}$ & -9.31850 & -0.43167 & -2.80044 & $\mathrm{H}$ & 1.58939 & 0.59155 & 3.31346 \\
\hline $\mathrm{C}$ & 7.13588 & -3.32700 & -1.52089 & $\mathrm{H}$ & 0.49117 & 0.50205 & 4.68778 \\
\hline $\mathrm{C}$ & -2.54357 & 0.94781 & 4.12293 & $\mathrm{H}$ & 4.71695 & 1.93857 & -2.58595 \\
\hline $\mathrm{C}$ & 5.75879 & 4.70316 & -0.06466 & $\mathrm{H}$ & 4.04121 & 4.30192 & -2.97314 \\
\hline $\mathrm{C}$ & 0.61915 & 0.20313 & 3.63791 & $\mathrm{H}$ & 0.22095 & -1.60436 & 2.51335 \\
\hline $\mathrm{C}$ & 5.01253 & 2.71353 & -1.88718 & $\mathrm{H}$ & -0.15566 & -1.74485 & 4.22304 \\
\hline $\mathrm{C}$ & 4.99522 & 5.02571 & -1.19416 & $\mathrm{H}$ & 2.64906 & -1.51612 & 3.05609 \\
\hline $\mathrm{C}$ & 4.63121 & 4.02334 & -2.10657 & $\mathrm{H}$ & 1.13638 & -3.96582 & 4.10778 \\
\hline $\mathrm{C}$ & 0.57330 & -1.32782 & 3.51381 & $\mathrm{H}$ & 2.83675 & -3.95672 & 3.61200 \\
\hline $\mathrm{C}$ & 1.93826 & -1.97944 & 3.75670 & $\mathrm{H}$ & 1.56872 & -3.64838 & 2.41143 \\
\hline $\mathrm{C}$ & 1.86606 & -3.47508 & 3.45153 & $\mathrm{H}$ & 3.40742 & -2.20800 & 5.34488 \\
\hline $\mathrm{C}$ & 2.43390 & -1.73410 & 5.18192 & $\mathrm{H}$ & 1.72774 & -2.15720 & 5.90747 \\
\hline $\mathrm{C}$ & 4.89567 & 7.31790 & -0.58973 & $\mathrm{H}$ & 2.54194 & -0.66632 & 5.3997 \\
\hline $\mathrm{H}$ & -1.87404 & 2.62191 & 1.63185 & $\mathrm{H}$ & 4.45423 & 8.22089 & -1.00856 \\
\hline $\mathrm{H}$ & 3.10175 & -3.80601 & 0.00872 & $\mathrm{H}$ & 5.98064 & 7.44187 & -0.51116 \\
\hline $\mathrm{H}$ & 8.16880 & -0.86212 & -0.45793 & $\mathrm{H}$ & 4.47407 & 7.12846 & 0.40282 \\
\hline
\end{tabular}


Structure, energy and Cartesian coordinate of $\mathbf{1 - B \mathbf { j }}$ :

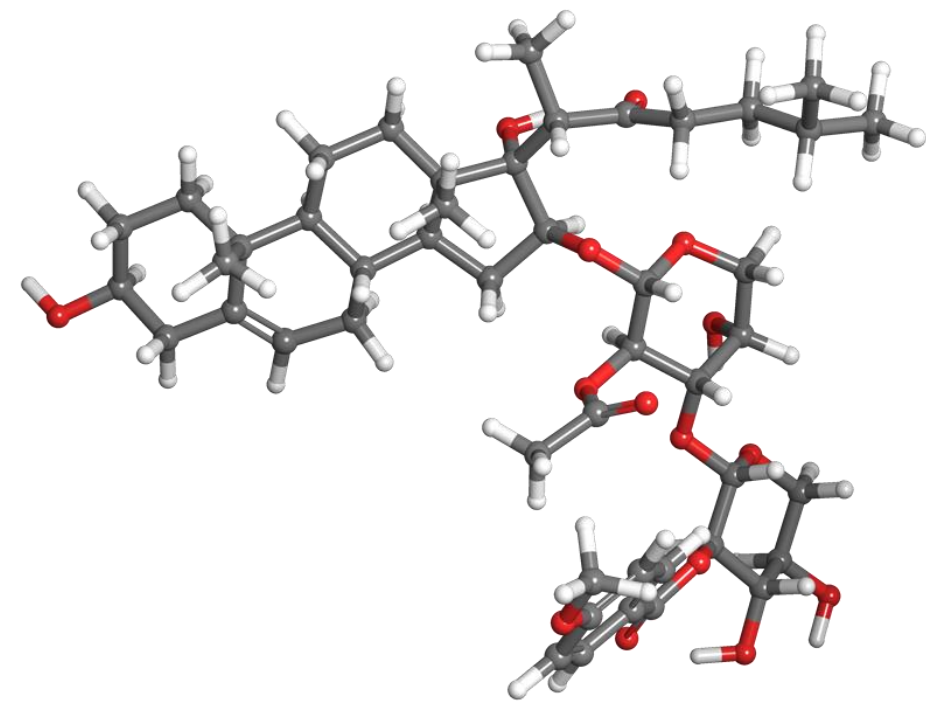

1-Bj $(\Delta G=2.7 \mathrm{kcal} / \mathrm{mol}$, Boltzmann pop. $(298 \mathrm{~K})=0.4 \%)$

M06-2X/6-311+G(d,p)-PCM(MeOH)//M06-2X/6-31G(d)-PCM(MeOH):

Gibbs Free Energy (a.u.) $=-2958.9925341$

M06-2X/6-311+G(d,p)-PCM(MeOH): $\quad$ Electronic energy (a.u. $)=-2960.0294561$

M06-2X/6-31G(d)-PCM(MeOH): Zero-point correction (a.u.) = 1.134337

Thermal correction to Energy (a.u.) $=1.194774$

Thermal correction to Enthalpy (a.u.) $=1.195718$

Thermal correction to Gibbs Free Energy (a.u.) $=1.036922$

$\begin{array}{lrrrrrrr}\mathrm{O} & -0.50721 & 1.99460 & 0.62051 & \mathrm{H} & 8.08001 & -2.37476 & -3.93440 \\ \mathrm{O} & 3.23645 & -0.25059 & -1.38843 & \mathrm{H} & -9.87542 & -4.92466 & -1.99117 \\ \mathrm{O} & 4.85874 & -2.18820 & -0.30309 & \mathrm{H} & 1.31830 & -2.82782 & 1.39353 \\ \mathrm{O} & 0.98118 & -0.43447 & 0.20380 & \mathrm{H} & -0.25057 & -2.06765 & 1.70320 \\ \mathrm{O} & -3.22701 & 3.17766 & -1.40681 & \mathrm{H} & 0.99222 & -2.12371 & 2.99668 \\ \mathrm{O} & 1.07809 & 3.14849 & -0.54892 & \mathrm{H} & 3.39448 & 0.94828 & 0.28620 \\ \mathrm{O} & 2.58686 & 2.09593 & -2.76691 & \mathrm{H} & -1.43818 & -0.07616 & -1.54831 \\ \mathrm{O} & 4.73220 & 0.17711 & -3.02571 & \mathrm{H} & -1.54316 & -0.20263 & 0.20867 \\ \mathrm{O} & 6.80701 & -3.34518 & -2.23519 & \mathrm{H} & -3.72910 & -1.38549 & -2.53841 \\ \mathrm{O} & 7.80119 & -1.45323 & -4.06604 & \mathrm{H} & -2.77876 & -2.26311 & -1.35691 \\ \mathrm{O} & -8.98934 & -5.13045 & -1.65789 & \mathrm{H} & -3.69032 & 0.82366 & -1.69544 \\ \mathrm{O} & -1.32536 & 5.06100 & -0.88801 & \mathrm{H} & -5.88618 & -0.27824 & -1.61206 \\ \mathrm{O} & 2.08796 & 0.03476 & 2.11106 & \mathrm{H} & 4.25405 & -2.38651 & -2.27301 \\ \mathrm{O} & 4.49795 & -4.32949 & -0.88405 & \mathrm{H} & -2.14275 & 3.42041 & 1.76988 \\ \mathrm{O} & 2.91132 & -4.37211 & 5.26235 & \mathrm{H} & -5.40275 & 2.27149 & -0.91660 \\ \mathrm{C} & 0.81731 & -2.01384 & 1.92745 & \mathrm{H} & -5.59166 & 2.66400 & 0.79187 \\ \mathrm{C} & 2.85611 & 0.91565 & -0.66940 & \mathrm{H} & -7.14845 & 0.87795 & 0.13148 \\ \mathrm{C} & -1.87741 & 0.38072 & -0.65582 & \mathrm{H} & -6.03813 & 0.34200 & 1.37448 \\ \mathrm{C} & -2.74802 & 2.66965 & -0.16989 & \mathrm{H} & 1.36744 & 2.18406 & 1.25085\end{array}$




\begin{tabular}{|c|c|c|c|c|c|c|c|}
\hline $\mathrm{C}$ & -3.78819 & -1.87975 & -1.55513 & $\mathrm{H}$ & -3.92809 & -1.27001 & 0.49228 \\
\hline $\mathrm{C}$ & -3.40337 & 0.44794 & -0.70201 & $\mathrm{H}$ & 0.86916 & 0.74286 & -1.40656 \\
\hline $\mathrm{C}$ & -1.35967 & 4.72273 & 0.28567 & $\mathrm{H}$ & 4.20776 & 2.38864 & -1.50526 \\
\hline $\mathrm{C}$ & -5.68958 & -0.56636 & -0.56478 & $\mathrm{H}$ & -4.43413 & -3.90581 & -2.17772 \\
\hline $\mathrm{C}$ & -3.75955 & 1.57909 & 0.28787 & $\mathrm{H}$ & -3.95713 & 0.26115 & 2.02920 \\
\hline $\mathrm{C}$ & 4.97139 & -1.81314 & -1.67489 & $\mathrm{H}$ & -3.83053 & 1.97316 & 2.42539 \\
\hline $\mathrm{C}$ & -2.47637 & 3.82960 & 0.81372 & $\mathrm{H}$ & -2.39149 & 1.07150 & 1.92936 \\
\hline $\mathrm{C}$ & -5.24917 & 1.88861 & 0.09824 & $\mathrm{H}$ & -0.98406 & 2.26904 & -1.36870 \\
\hline $\mathrm{C}$ & -6.09828 & 0.62685 & 0.31812 & $\mathrm{H}$ & 5.20468 & 0.22979 & -1.01180 \\
\hline $\mathrm{C}$ & 0.84100 & 2.04439 & 0.29786 & $\mathrm{H}$ & 7.07735 & -1.45255 & -1.53996 \\
\hline $\mathrm{C}$ & -4.17557 & -0.84942 & -0.49349 & $\mathrm{H}$ & -8.99937 & -3.24074 & 0.20044 \\
\hline $\mathrm{C}$ & 1.37645 & -0.69797 & 1.46172 & $\mathrm{H}$ & -9.86061 & -2.59036 & -1.19559 \\
\hline $\mathrm{C}$ & -5.96584 & -3.03335 & -1.05758 & $\mathrm{H}$ & -5.57178 & -2.16829 & 1.64246 \\
\hline $\mathrm{C}$ & 1.35630 & 0.79037 & -0.42810 & $\mathrm{H}$ & -6.99514 & -3.17624 & 1.38637 \\
\hline $\mathrm{C}$ & 3.12404 & 2.20120 & -1.46853 & $\mathrm{H}$ & -7.19763 & -1.46633 & 1.76144 \\
\hline $\mathrm{C}$ & -4.75677 & -3.02299 & -1.62548 & $\mathrm{H}$ & -8.45820 & -0.81885 & -0.18351 \\
\hline $\mathrm{C}$ & -3.46372 & 1.19442 & 1.74656 & $\mathrm{H}$ & -7.92824 & -1.19151 & -1.82230 \\
\hline $\mathrm{C}$ & -1.43540 & 1.84759 & -0.46535 & $\mathrm{H}$ & 6.75919 & 0.63638 & -2.86454 \\
\hline $\mathrm{C}$ & -6.54059 & -1.84008 & -0.29415 & $\mathrm{H}$ & 6.06965 & 0.54677 & -4.50566 \\
\hline $\mathrm{C}$ & 4.57627 & -0.34047 & -1.71752 & $\mathrm{H}$ & 2.96754 & 3.57358 & 0.19295 \\
\hline $\mathrm{C}$ & 6.38431 & -1.99806 & -2.19782 & $\mathrm{H}$ & 2.53401 & 4.26683 & -1.39343 \\
\hline $\mathrm{C}$ & -8.86319 & -2.84152 & -0.81295 & $\mathrm{H}$ & 3.68405 & -5.73125 & 1.06882 \\
\hline $\mathrm{C}$ & -6.57813 & -2.18096 & 1.21004 & $\mathrm{H}$ & -8.14499 & -3.55159 & -2.70935 \\
\hline $\mathrm{C}$ & -7.98411 & -1.59038 & -0.79958 & $\mathrm{H}$ & -6.97546 & -4.72755 & -0.16834 \\
\hline $\mathrm{C}$ & 6.06680 & 0.08525 & -3.51758 & $\mathrm{H}$ & -6.37411 & -5.01027 & -1.80301 \\
\hline $\mathrm{C}$ & 2.46136 & 3.37724 & -0.76462 & $\mathrm{H}$ & 5.78395 & -1.91176 & -4.25938 \\
\hline $\mathrm{C}$ & 3.70196 & -4.91993 & 1.78937 & $\mathrm{H}$ & -4.44786 & 4.20973 & 1.65955 \\
\hline $\mathrm{C}$ & -8.23513 & -3.92736 & -1.67646 & $\mathrm{H}$ & -4.16164 & 4.99508 & 0.09205 \\
\hline $\mathrm{C}$ & 4.12912 & -3.64928 & 1.37798 & $\mathrm{H}$ & -3.42327 & 5.64285 & 1.57187 \\
\hline $\mathrm{C}$ & 4.50922 & -3.45745 & -0.03510 & $\mathrm{H}$ & 2.95978 & -6.10262 & 3.43220 \\
\hline $\mathrm{C}$ & -6.84323 & -4.26195 & -1.15572 & $\mathrm{H}$ & -0.90503 & 5.68661 & 2.11295 \\
\hline $\mathrm{C}$ & 6.47826 & -1.37969 & -3.58987 & $\mathrm{H}$ & 0.12654 & 4.32932 & 1.71775 \\
\hline $\mathrm{C}$ & -3.70409 & 4.72377 & 1.04684 & $\mathrm{H}$ & 4.45781 & -1.60772 & 1.97985 \\
\hline $\mathrm{C}$ & 3.30005 & -5.13007 & 3.09336 & $\mathrm{H}$ & 3.76622 & -1.96596 & 4.30438 \\
\hline $\mathrm{C}$ & -0.34780 & 5.22233 & 1.28700 & $\mathrm{H}$ & 0.21207 & 7.11566 & 0.42336 \\
\hline $\mathrm{C}$ & 4.14710 & -2.59751 & 2.29565 & $\mathrm{H}$ & 1.04724 & 5.72308 & -0.24598 \\
\hline $\mathrm{C}$ & 3.32430 & -4.07035 & 4.01492 & $\mathrm{H}$ & 2.33141 & 5.46623 & 1.87520 \\
\hline $\mathrm{C}$ & 3.75278 & -2.79966 & 3.61309 & $\mathrm{H}$ & 0.92875 & 8.06256 & 2.69174 \\
\hline $\mathrm{C}$ & 0.68925 & 6.16275 & 0.69149 & $\mathrm{H}$ & 2.31397 & 7.35548 & 3.54042 \\
\hline $\mathrm{C}$ & 1.87805 & 6.43584 & 1.61736 & $\mathrm{H}$ & 0.76982 & 6.49540 & 3.50486 \\
\hline $\mathrm{C}$ & 1.44651 & 7.12116 & 2.91469 & $\mathrm{H}$ & 3.79997 & 7.45920 & 1.52611 \\
\hline $\mathrm{C}$ & 2.92755 & 7.27601 & 0.89046 & $\mathrm{H}$ & 2.50781 & 8.24904 & 0.60723 \\
\hline $\mathrm{C}$ & 2.89822 & -3.33131 & 6.22815 & $\mathrm{H}$ & 3.26911 & 6.77880 & -0.02401 \\
\hline $\mathrm{H}$ & -2.62363 & 3.90834 & -1.64162 & $\mathrm{H}$ & 2.53677 & -3.78337 & 7.15046 \\
\hline $\mathrm{H}$ & 3.10042 & 1.39859 & -3.21242 & $\mathrm{H}$ & 2.22233 & -2.52477 & 5.92449 \\
\hline $\mathrm{H}$ & 6.02651 & -3.91914 & -2.13795 & $\mathrm{H}$ & 3.90408 & -2.92871 & 6.38525 \\
\hline
\end{tabular}


Structure, energy and Cartesian coordinate of 1-Bk:

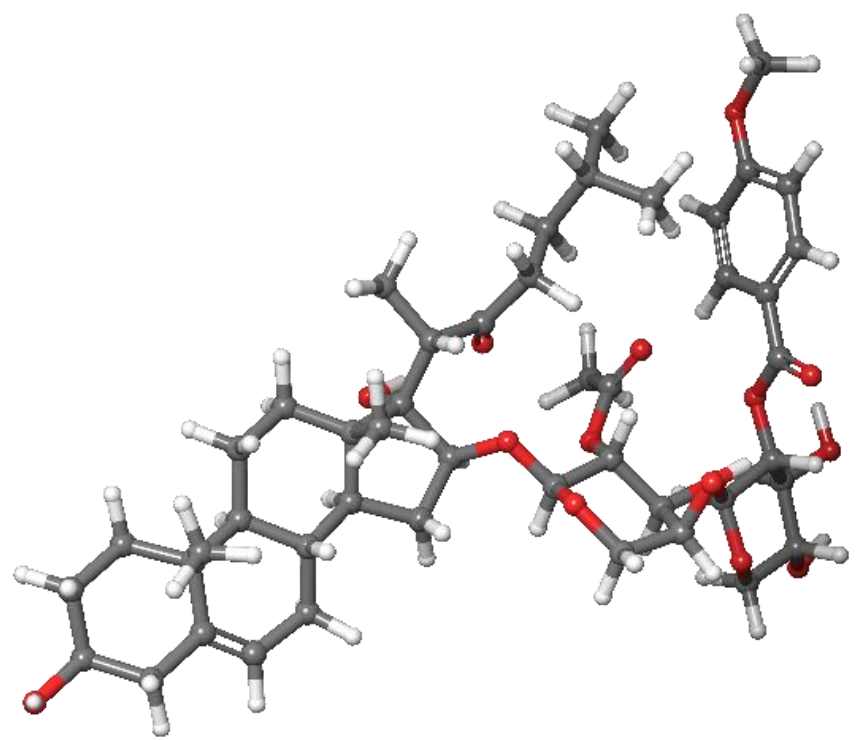

1-Bk $(\Delta G=2.7 \mathrm{kcal} / \mathrm{mol}$, Boltzmann pop. $(298 \mathrm{~K})=0.3 \%)$

M06-2X/6-311+G(d,p)-PCM(MeOH)//M06-2X/6-31G(d)-PCM(MeOH):

Gibbs Free Energy (a.u.) $=-2958.9903787$

M06-2X/6-311+G(d,p)-PCM(MeOH): $\quad$ Electronic energy (a.u. $)=-2960.0314027$

M06-2X/6-31G(d)-PCM(MeOH): Zero-point correction $($ a.u. $)=1.135150$

Thermal correction to Energy (a.u.) $=1.195332$

Thermal correction to Enthalpy (a.u.) $=1.196276$

Thermal correction to Gibbs Free Energy (a.u.) $=1.041024$

$\begin{array}{lrrrrrrr}\mathrm{O} & 0.96071 & 0.01422 & 0.78528 & \mathrm{H} & -6.63113 & 5.53366 & -2.64085 \\ \mathrm{O} & -3.07123 & 2.60003 & 1.05390 & \mathrm{H} & 12.44799 & 1.07431 & 0.16261 \\ \mathrm{O} & -5.27627 & 1.52082 & -0.41037 & \mathrm{H} & -3.27705 & 0.73770 & -2.58093 \\ \mathrm{O} & -1.34447 & 0.95092 & -0.53812 & \mathrm{H} & -2.98597 & -1.01568 & -2.52694 \\ \mathrm{O} & 2.55893 & -1.67005 & -1.90932 & \mathrm{H} & -1.63145 & 0.11233 & -2.84717 \\ \mathrm{O} & 0.67554 & 1.54974 & 2.40865 & \mathrm{H} & -1.23798 & 3.36616 & 0.46377 \\ \mathrm{O} & -1.95632 & 2.14002 & 3.49157 & \mathrm{H} & 3.08878 & 1.82466 & -0.64351 \\ \mathrm{O} & -3.40921 & 4.62195 & 0.07590 & \mathrm{H} & 3.15253 & 1.19192 & 1.00836 \\ \mathrm{O} & -7.02855 & 3.50665 & -1.49934 & \mathrm{H} & 5.73529 & 1.85435 & -1.60095 \\ \mathrm{O} & -5.72148 & 5.81570 & -2.45094 & \mathrm{H} & 5.46720 & 2.60329 & -0.03977 \\ \mathrm{O} & 12.19489 & 1.23926 & -0.75916 & \mathrm{H} & 4.34827 & -0.05212 & -1.50812 \\ \mathrm{O} & -0.17244 & -1.83842 & -1.48386 & \mathrm{H} & 6.74895 & -0.53880 & -1.51612 \\ \mathrm{O} & -2.95539 & -0.52282 & 0.03302 & \mathrm{H} & -5.56013 & 3.22658 & 0.72765 \\ \mathrm{O} & -5.72115 & 1.22083 & 1.78201 & \mathrm{H} & 1.44405 & -2.36944 & 1.18810 \\ \mathrm{O} & -7.37111 & -4.38353 & -0.76342 & \mathrm{H} & 4.83503 & -2.38555 & -1.58018 \\ \mathrm{C} & -2.55858 & -0.03987 & -2.29547 & \mathrm{H} & 4.67673 & -3.41427 & -0.15684 \\ \mathrm{C} & -1.65248 & 2.62334 & 1.15744 & \mathrm{H} & 7.02024 & -2.74469 & -0.47687 \\ \mathrm{C} & 3.12198 & 0.90591 & -0.05107 & \mathrm{H} & 6.38934 & -2.15902 & 1.04852 \\ \mathrm{C} & 2.43804 & -1.45559 & -0.50904 & \mathrm{H} & 0.87520 & 2.03585 & 0.41341\end{array}$




\begin{tabular}{|c|c|c|c|c|c|c|c|}
\hline $\mathrm{C}$ & 6.04673 & 1.81947 & -0.54448 & $\mathrm{H}$ & 5.72345 & 0.53531 & 1.13749 \\
\hline $\mathrm{C}$ & 4.31012 & 0.01266 & -0.41008 & $\mathrm{H}$ & -1.54350 & 0.48924 & 1.47523 \\
\hline $\mathrm{C}$ & 0.08387 & -2.37934 & -0.41667 & $\mathrm{H}$ & -1.56198 & 4.04849 & 2.76447 \\
\hline $\mathrm{C}$ & 6.73818 & -0.59511 & -0.41366 & $\mathrm{H}$ & 7.80039 & 3.17161 & -0.61044 \\
\hline $\mathrm{C}$ & 3.88094 & -1.38955 & 0.07168 & $\mathrm{H}$ & 4.74297 & -1.14879 & 2.07384 \\
\hline $\mathrm{C}$ & -5.06030 & 2.91298 & -0.19276 & $\mathrm{H}$ & 3.63200 & -2.50738 & 1.92423 \\
\hline $\mathrm{C}$ & 1.50423 & -2.51456 & 0.10532 & $\mathrm{H}$ & 3.00231 & -0.85634 & 2.01211 \\
\hline $\mathrm{C}$ & 4.89658 & -2.39331 & -0.48614 & $\mathrm{H}$ & 1.35713 & 0.29037 & -1.20884 \\
\hline $\mathrm{C}$ & 6.31925 & -2.02677 & -0.03714 & $\mathrm{H}$ & -3.03853 & 2.89356 & -0.97894 \\
\hline $\mathrm{C}$ & 0.41741 & 1.26274 & 1.05493 & $\mathrm{H}$ & -5.13359 & 3.27548 & -2.30898 \\
\hline $\mathrm{C}$ & 5.69917 & 0.44976 & 0.04128 & $\mathrm{H}$ & 10.95900 & -0.83267 & 0.44553 \\
\hline $\mathrm{C}$ & -2.32504 & 0.06112 & -0.81613 & $\mathrm{H}$ & 11.30143 & -1.24240 & -1.23569 \\
\hline $\mathrm{C}$ & 8.46302 & 1.24315 & -0.15462 & $\mathrm{H}$ & 7.55918 & -0.18671 & 2.15597 \\
\hline $\mathrm{C}$ & -1.09093 & 1.24780 & 0.83362 & $\mathrm{H}$ & 9.30815 & -0.15731 & 1.93750 \\
\hline $\mathrm{C}$ & -1.28673 & 2.99809 & 2.59023 & $\mathrm{H}$ & 8.43049 & -1.66592 & 1.70493 \\
\hline $\mathrm{C}$ & 7.51043 & 2.13416 & -0.44284 & $\mathrm{H}$ & 9.07450 & -2.12068 & -0.58121 \\
\hline $\mathrm{C}$ & 3.81029 & -1.47354 & 1.60605 & $\mathrm{H}$ & 8.93327 & -0.91249 & -1.85643 \\
\hline $\mathrm{C}$ & 1.89379 & 0.00032 & -0.29823 & $\mathrm{H}$ & -3.29705 & 5.00634 & -1.96825 \\
\hline $\mathrm{C}$ & 8.17813 & -0.24376 & 0.05724 & $\mathrm{H}$ & -3.61019 & 6.39206 & -0.89258 \\
\hline $\mathrm{C}$ & -3.57516 & 3.22811 & -0.07336 & $\mathrm{H}$ & 0.74230 & 3.61531 & 2.21768 \\
\hline $\mathrm{C}$ & -5.62846 & 3.64881 & -1.39756 & $\mathrm{H}$ & 0.46085 & 2.95054 & 3.84793 \\
\hline $\mathrm{C}$ & 10.64676 & -0.64474 & -0.59232 & $\mathrm{H}$ & -5.45352 & -0.47329 & -1.78499 \\
\hline $\mathrm{C}$ & 8.37963 & -0.58354 & 1.54850 & $\mathrm{H}$ & 10.59965 & 1.01144 & -1.95785 \\
\hline $\mathrm{C}$ & 9.18736 & -1.05263 & -0.79625 & $\mathrm{H}$ & 10.24945 & 1.57562 & 1.00841 \\
\hline $\mathrm{C}$ & -3.83960 & 5.34063 & -1.07103 & $\mathrm{H}$ & 10.00940 & 2.73492 & -0.29904 \\
\hline $\mathrm{C}$ & 0.21498 & 2.83808 & 2.79048 & $\mathrm{H}$ & -5.87497 & 5.52297 & -0.39870 \\
\hline $\mathrm{C}$ & -5.93557 & -1.08337 & -1.02927 & $\mathrm{H}$ & 2.81312 & -4.22564 & 0.44814 \\
\hline $\mathrm{C}$ & 10.84171 & 0.83386 & -0.90223 & $\mathrm{H}$ & 2.25438 & -4.05416 & -1.22858 \\
\hline $\mathrm{C}$ & -6.10879 & -0.57908 & 0.26637 & $\mathrm{H}$ & 1.16247 & -4.67549 & 0.02631 \\
\hline $\mathrm{C}$ & -5.67615 & 0.78231 & 0.65569 & $\mathrm{H}$ & -6.24018 & -2.77238 & -2.33543 \\
\hline $\mathrm{C}$ & 9.90742 & 1.67758 & -0.03341 & $\mathrm{H}$ & -0.57375 & -3.69197 & 1.15017 \\
\hline $\mathrm{C}$ & -5.33624 & 5.13732 & -1.27820 & $\mathrm{H}$ & -1.46392 & -2.17977 & 0.98646 \\
\hline $\mathrm{C}$ & 1.96178 & -3.95638 & -0.18021 & $\mathrm{H}$ & -6.86489 & -0.96769 & 2.23098 \\
\hline $\mathrm{C}$ & -6.37079 & -2.35744 & -1.34113 & $\mathrm{H}$ & -7.66634 & -3.24045 & 1.69564 \\
\hline $\mathrm{C}$ & -1.00990 & -3.00256 & 0.41782 & $\mathrm{H}$ & -1.56508 & -4.37465 & -1.14696 \\
\hline $\mathrm{C}$ & -6.73218 & -1.36803 & 1.23102 & $\mathrm{H}$ & -2.59968 & -2.94974 & -1.02958 \\
\hline $\mathrm{C}$ & -6.99402 & -3.14943 & -0.36326 & $\mathrm{H}$ & -2.53200 & -5.25699 & 0.96526 \\
\hline $\mathrm{C}$ & -7.18504 & -2.64740 & 0.92772 & $\mathrm{H}$ & -4.61265 & -4.50412 & -1.15057 \\
\hline $\mathrm{C}$ & -2.06646 & -3.70330 & -0.43649 & $\mathrm{H}$ & -4.77644 & -5.82226 & 0.01770 \\
\hline $\mathrm{C}$ & -3.08195 & -4.50143 & 0.38445 & $\mathrm{H}$ & -3.51803 & -5.89796 & -1.23120 \\
\hline $\mathrm{C}$ & -4.05090 & -5.22724 & -0.54815 & $\mathrm{H}$ & -4.64851 & -4.13982 & 1.85315 \\
\hline $\mathrm{C}$ & -3.84026 & -3.59417 & 1.35316 & $\mathrm{H}$ & -4.28250 & -2.74986 & 0.81051 \\
\hline $\mathrm{C}$ & -7.91913 & -5.25328 & 0.21444 & $\mathrm{H}$ & -3.18219 & -3.18621 & 2.12772 \\
\hline $\mathrm{H}$ & 1.64556 & -1.64220 & -2.24832 & $\mathrm{H}$ & -8.11112 & -6.19643 & -0.29533 \\
\hline $\mathrm{H}$ & -2.89250 & 2.13994 & 3.22882 & $\mathrm{H}$ & -8.85701 & -4.85530 & 0.61557 \\
\hline $\mathrm{H}$ & -7.22040 & 2.58858 & -1.74479 & $\mathrm{H}$ & -7.20900 & -5.41510 & 1.03315 \\
\hline
\end{tabular}


Structure, energy and Cartesian coordinate of 1-Bl:

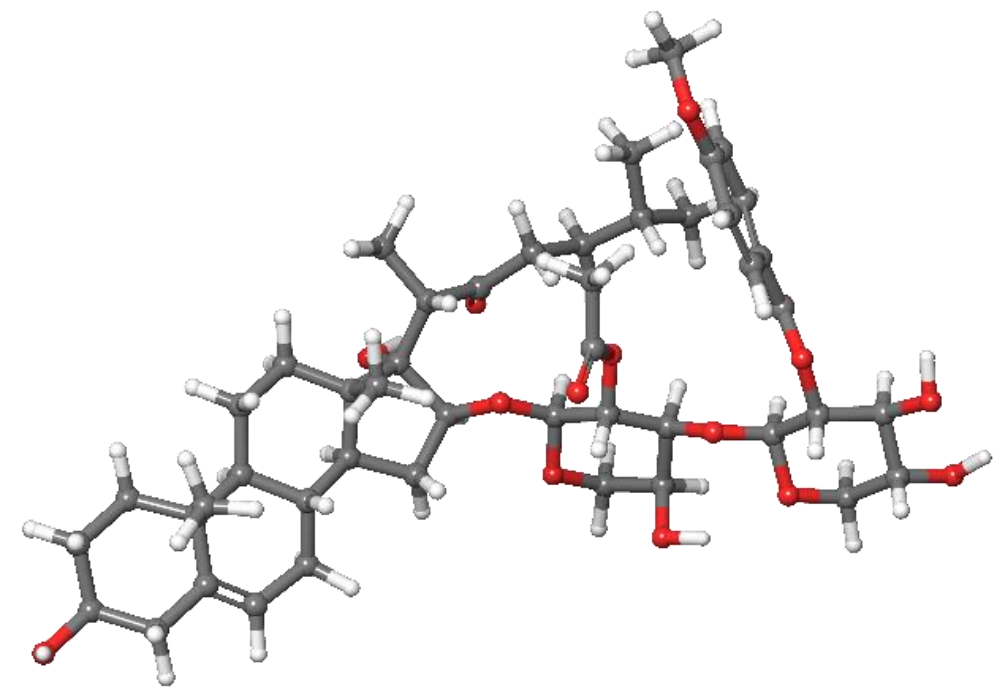

1-BI $(\Delta G=2.8 \mathrm{kcal} / \mathrm{mol}$, Boltzmann pop. $(298 \mathrm{~K})=0.3 \%)$

M06-2X/6-311+G(d,p)-PCM(MeOH)//M06-2X/6-31G(d)-PCM(MeOH):

Gibbs Free Energy (a.u.) = -2958.9902243

M06-2X/6-311+G(d,p)-PCM(MeOH): $\quad$ Electronic energy (a.u. $)=-2960.0306033$

M06-2X/6-31G(d)-PCM(MeOH): Zero-point correction (a.u.) $=1.135074$

Thermal correction to Energy (a.u.) $=1.195344$

Thermal correction to Enthalpy (a.u.) $=1.196289$

Thermal correction to Gibbs Free Energy (a.u.) = 1.040379

$\begin{array}{lrrr}\mathrm{O} & 0.84599 & -0.24912 & 0.73340 \\ \mathrm{O} & -3.31907 & -2.12406 & -0.40950 \\ \mathrm{O} & -5.55291 & -0.94556 & -1.61940 \\ \mathrm{O} & -1.75201 & 0.17527 & -0.18799 \\ \mathrm{O} & 3.31956 & 1.22061 & 2.91410 \\ \mathrm{O} & 0.25144 & -2.31183 & 1.53548 \\ \mathrm{O} & -1.57351 & -4.13532 & 0.22700 \\ \mathrm{O} & -4.46291 & -4.07316 & -0.05629 \\ \mathrm{O} & -7.87139 & -2.67331 & -1.84439 \\ \mathrm{O} & -8.00730 & -4.84390 & -0.03119 \\ \mathrm{O} & 11.77414 & -1.69380 & -1.82679 \\ \mathrm{O} & 0.66486 & 1.49221 & 3.56214 \\ \mathrm{O} & -0.78225 & 0.05058 & -2.22310 \\ \mathrm{O} & -5.65531 & -0.15534 & 0.49259 \\ \mathrm{O} & -4.80939 & 5.11218 & -2.99535 \\ \mathrm{C} & -1.83851 & 2.10530 & -1.54290 \\ \mathrm{C} & -2.44629 & -1.93056 & 0.69578 \\ \mathrm{C} & 3.10398 & -1.19105 & 0.86702 \\ \mathrm{C} & 2.69327 & 1.14591 & 1.63986 \\ \mathrm{C} & 5.95872 & -2.15675 & 0.18698\end{array}$

$\begin{array}{rrrr}\mathrm{H} & -8.72092 & -4.53748 & -0.61389 \\ \mathrm{H} & 11.67848 & -1.48668 & -2.76945 \\ \mathrm{H} & -1.01896 & 2.75178 & -1.20897 \\ \mathrm{H} & -2.72264 & 2.31984 & -0.93801 \\ \mathrm{H} & -2.03557 & 2.31557 & -2.59407 \\ \mathrm{H} & -2.96548 & -1.34831 & 1.47363 \\ \mathrm{H} & 3.24495 & -2.12160 & 1.42329 \\ \mathrm{H} & 2.75645 & -1.45766 & -0.13801 \\ \mathrm{H} & 6.05761 & -2.25741 & 1.27997 \\ \mathrm{H} & 5.20263 & -2.89519 & -0.11000 \\ \mathrm{H} & 4.80814 & -0.31915 & 1.80869 \\ \mathrm{H} & 7.05832 & 0.11700 & 0.96599 \\ \mathrm{H} & -5.19584 & -2.84225 & -2.10933 \\ \mathrm{H} & 1.21527 & 2.24060 & 0.49473 \\ \mathrm{H} & 5.34220 & 1.98795 & 1.83401 \\ \mathrm{H} & 4.72211 & 3.10553 & 0.61824 \\ \mathrm{H} & 7.00666 & 2.38722 & 0.05105 \\ \mathrm{H} & 5.85629 & 1.92180 & -1.18423 \\ \mathrm{H} & -0.59728 & -0.55044 & 2.16295 \\ \mathrm{H} & 5.09410 & -0.75853 & -1.18298\end{array}$




\begin{tabular}{|c|c|c|c|c|c|c|c|}
\hline $\mathrm{C}$ & 4.36321 & -0.32534 & 0.80269 & $\mathrm{H}$ & -0.84197 & -1.61232 & -0.70842 \\
\hline $\mathrm{C}$ & 0.48425 & 2.01127 & 2.46955 & $\mathrm{H}$ & -2.77478 & -3.72226 & 1.86587 \\
\hline $\mathrm{C}$ & 6.64506 & 0.25142 & -0.04856 & $\mathrm{H}$ & 7.56054 & -3.53463 & -0.48109 \\
\hline $\mathrm{C}$ & 3.83084 & 1.10830 & 0.57962 & $\mathrm{H}$ & 3.91879 & 1.00124 & -1.60860 \\
\hline $\mathrm{C}$ & -5.53727 & -2.31396 & -1.21386 & $\mathrm{H}$ & 2.97212 & 2.35048 & -0.98652 \\
\hline $\mathrm{C}$ & 1.63719 & 2.26303 & 1.50341 & $\mathrm{H}$ & 2.30554 & 0.71093 & -0.95345 \\
\hline $\mathrm{C}$ & 5.01083 & 2.06324 & 0.79229 & $\mathrm{H}$ & 1.78887 & -0.61796 & 2.52990 \\
\hline $\mathrm{C}$ & 6.16899 & 1.71164 & -0.15531 & $\mathrm{H}$ & -4.90033 & -2.29983 & 0.88483 \\
\hline $\mathrm{C}$ & -0.19616 & -1.01014 & 1.23769 & $\mathrm{H}$ & -7.24294 & -2.34436 & 0.10795 \\
\hline $\mathrm{C}$ & 5.47450 & -0.74680 & -0.15110 & $\mathrm{H}$ & 10.27300 & 0.47837 & -2.38588 \\
\hline $\mathrm{C}$ & -1.39868 & 0.67452 & -1.39535 & $\mathrm{H}$ & 11.21671 & 0.76704 & -0.92395 \\
\hline $\mathrm{C}$ & 8.08082 & -1.58859 & -1.03419 & $\mathrm{H}$ & 6.45214 & 0.00446 & -2.76415 \\
\hline $\mathrm{C}$ & -1.27635 & -1.12099 & 0.16482 & $\mathrm{H}$ & 8.15868 & -0.05022 & -3.20281 \\
\hline $\mathrm{C}$ & -1.96402 & -3.27571 & 1.27294 & $\mathrm{H}$ & 7.47240 & 1.44429 & -2.57116 \\
\hline $\mathrm{C}$ & 7.26806 & -2.48454 & -0.46742 & $\mathrm{H}$ & 8.94017 & 1.72924 & -0.67717 \\
\hline $\mathrm{C}$ & 3.22163 & 1.29563 & -0.82013 & $\mathrm{H}$ & 9.22512 & 0.44642 & 0.49720 \\
\hline $\mathrm{C}$ & 2.04577 & -0.28072 & 1.52164 & $\mathrm{H}$ & -5.93486 & -4.26803 & 1.40080 \\
\hline $\mathrm{C}$ & 7.79727 & -0.08590 & -1.03858 & $\mathrm{H}$ & -5.51557 & -5.72923 & 0.47164 \\
\hline $\mathrm{C}$ & -4.55317 & -2.65486 & -0.09380 & $\mathrm{H}$ & -1.08229 & -2.54716 & 3.10539 \\
\hline $\mathrm{C}$ & -6.91413 & -2.83619 & -0.81956 & $\mathrm{H}$ & -0.32766 & -4.02404 & 2.44651 \\
\hline $\mathrm{C}$ & 10.34915 & 0.22665 & -1.31793 & $\mathrm{H}$ & -5.70385 & 2.36105 & 0.54403 \\
\hline $\mathrm{C}$ & 7.44938 & 0.35387 & -2.47554 & $\mathrm{H}$ & 10.72672 & -1.51525 & -0.12222 \\
\hline $\mathrm{C}$ & 9.08070 & 0.64775 & -0.57364 & $\mathrm{H}$ & 9.30897 & -1.86881 & -2.78817 \\
\hline $\mathrm{C}$ & -5.67920 & -4.65328 & 0.40298 & $\mathrm{H}$ & 9.51057 & -3.11222 & -1.55305 \\
\hline $\mathrm{C}$ & -0.76583 & -3.05962 & 2.18509 & $\mathrm{H}$ & -6.58214 & -4.81282 & -1.54159 \\
\hline $\mathrm{C}$ & -5.47588 & 2.49603 & -0.50927 & $\mathrm{H}$ & 2.82910 & 3.99323 & 0.94186 \\
\hline $\mathrm{C}$ & 10.58094 & -1.27269 & -1.18274 & $\mathrm{H}$ & 2.80839 & 3.65909 & 2.68531 \\
\hline $\mathrm{C}$ & -5.36849 & 1.36904 & -1.32329 & $\mathrm{H}$ & 1.40168 & 4.40072 & 1.89243 \\
\hline $\mathrm{C}$ & -5.53794 & 0.03715 & -0.69971 & $\mathrm{H}$ & -5.38524 & 4.63402 & -0.37609 \\
\hline $\mathrm{C}$ & 9.36292 & -2.03744 & -1.70089 & $\mathrm{H}$ & -0.85459 & 3.52082 & 1.82250 \\
\hline $\mathrm{C}$ & -6.80964 & -4.34011 & -0.57331 & $\mathrm{H}$ & -1.06808 & 1.96280 & 1.05433 \\
\hline $\mathrm{C}$ & 2.20390 & 3.66560 & 1.77523 & $\mathrm{H}$ & -5.00386 & 0.65724 & -3.32780 \\
\hline $\mathrm{C}$ & -5.29685 & 3.77335 & -1.02790 & $\mathrm{H}$ & -4.68578 & 2.94002 & -4.26753 \\
\hline $\mathrm{C}$ & -0.89092 & 2.44095 & 2.02454 & $\mathrm{H}$ & -1.88025 & 2.63409 & 3.93426 \\
\hline $\mathrm{C}$ & -5.08792 & 1.52865 & -2.68805 & $\mathrm{H}$ & -1.91959 & 1.01597 & 3.25508 \\
\hline $\mathrm{C}$ & -5.00729 & 3.92363 & -2.38962 & $\mathrm{H}$ & -3.50813 & 1.76306 & 1.49893 \\
\hline $\mathrm{C}$ & -4.90941 & 2.79348 & -3.21613 & $\mathrm{H}$ & -3.32725 & 4.44304 & 2.97391 \\
\hline $\mathrm{C}$ & -2.00691 & 2.07906 & 2.99482 & $\mathrm{H}$ & -4.65779 & 4.04255 & 1.88213 \\
\hline $\mathrm{C}$ & -3.40612 & 2.34398 & 2.42943 & $\mathrm{H}$ & -3.01021 & 4.15122 & 1.25107 \\
\hline $\mathrm{C}$ & -3.60835 & 3.82741 & 2.11012 & $\mathrm{H}$ & -5.47797 & 1.98289 & 2.99833 \\
\hline $\mathrm{C}$ & -4.47115 & 1.85558 & 3.41046 & $\mathrm{H}$ & -4.41772 & 2.42137 & 4.34866 \\
\hline $\mathrm{C}$ & -4.89367 & 6.28532 & -2.19973 & $\mathrm{H}$ & -4.33479 & 0.79469 & 3.64621 \\
\hline $\mathrm{H}$ & 2.58972 & 1.21025 & 3.56036 & $\mathrm{H}$ & -4.69751 & 7.11805 & -2.87336 \\
\hline $\mathrm{H}$ & -2.38518 & -4.32531 & -0.27446 & $\mathrm{H}$ & -4.14283 & 6.27229 & -1.40241 \\
\hline $\mathrm{H}$ & -8.07773 & -1.73029 & -1.92689 & $\mathrm{H}$ & -5.89179 & 6.39252 & -1.76298 \\
\hline
\end{tabular}


Structure, energy and Cartesian coordinate of 1-C:

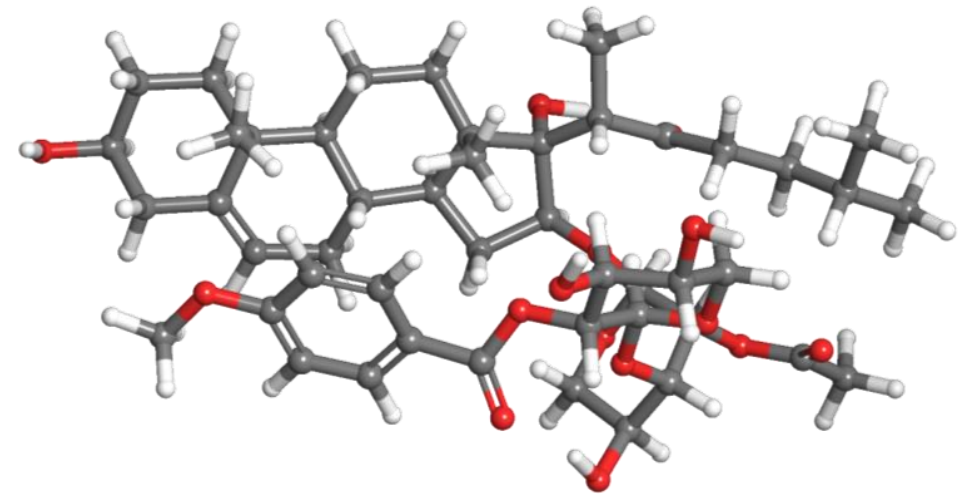

1-C $(\Delta G=0.0 \mathrm{kcal} / \mathrm{mol}$, Boltzmann pop. $(298 \mathrm{~K})=33.3 \%)$

M06-2X/6-311+G(d,p)-PCM(MeOH)//M06-2X/6-31G(d)-PCM(MeOH):

Gibbs Free Energy (a.u.) = -2958.9947086

M06-2X/6-311+G(d,p)-PCM(MeOH): $\quad$ Electronic energy (a.u. $)=-2960.0390066$

M06-2X/6-31G(d)-PCM(MeOH): Zero-point correction $($ a.u. $)=1.135415$

Thermal correction to Energy (a.u.) $=1.195162$

Thermal correction to Enthalpy (a.u.) $=1.196106$

Thermal correction to Gibbs Free Energy (a.u.) $=1.044298$

$\begin{array}{lrrrrrrr}\mathrm{O} & -1.69050 & -0.86385 & -0.83987 & \mathrm{H} & -4.59593 & 2.98235 & 4.70477 \\ \mathrm{O} & -2.58863 & 1.89679 & -0.83881 & \mathrm{H} & 9.95356 & -0.44133 & 0.06863 \\ \mathrm{O} & -0.52840 & 2.46368 & 0.99742 & \mathrm{H} & -6.82070 & -1.85237 & -3.51188 \\ \mathrm{O} & -4.74303 & -0.49174 & -2.63319 & \mathrm{H} & -6.62718 & -0.34759 & -4.41570 \\ \mathrm{O} & 0.04623 & -3.85625 & -0.11167 & \mathrm{H} & -7.96468 & -0.50602 & -3.23592 \\ \mathrm{O} & -1.94428 & -0.59704 & -3.14948 & \mathrm{H} & -4.45333 & 1.94440 & -1.71958 \\ \mathrm{O} & -2.40349 & 3.04193 & -3.18051 & \mathrm{H} & 0.89035 & -0.72971 & -2.38504 \\ \mathrm{O} & -4.14043 & 1.90288 & 0.83447 & \mathrm{H} & 0.60294 & 0.18928 & -0.89666 \\ \mathrm{O} & -1.41728 & 3.69966 & 3.34722 & \mathrm{H} & 3.58730 & -1.47667 & -2.52490 \\ \mathrm{O} & -3.75718 & 2.59594 & 4.41399 & \mathrm{H} & 3.22777 & 0.22709 & -2.30433 \\ \mathrm{O} & 9.79710 & -1.06906 & -0.65406 & \mathrm{H} & 1.98652 & -2.52349 & -1.10722 \\ \mathrm{O} & -2.70520 & -3.67243 & -0.22342 & \mathrm{H} & 4.25173 & -2.76795 & -0.36524 \\ \mathrm{O} & -6.40714 & 0.33942 & -1.36710 & \mathrm{H} & -2.15999 & 3.73061 & 0.83065 \\ \mathrm{O} & -0.45966 & 3.64354 & -0.93625 & \mathrm{H} & -1.44226 & -1.45458 & 1.71391 \\ \mathrm{O} & 5.69458 & 2.68063 & 0.27475 & \mathrm{H} & 2.17849 & -3.85791 & 0.86130 \\ \mathrm{C} & -6.92980 & -0.76592 & -3.45273 & \mathrm{H} & 1.76096 & -3.23226 & 2.45431 \\ \mathrm{C} & -3.50731 & 1.39826 & -1.79525 & \mathrm{H} & 4.19410 & -3.10637 & 2.07494 \\ \mathrm{C} & 0.72898 & -0.82142 & -1.30665 & \mathrm{H} & 3.50338 & -1.51563 & 2.31753 \\ \mathrm{C} & -0.17791 & -2.51393 & 0.30319 & \mathrm{H} & -2.86535 & -2.00337 & -2.08541 \\ \mathrm{C} & 3.77089 & -0.61688 & -1.85999 & \mathrm{H} & 3.14185 & 0.03412 & 0.07625 \\ \mathrm{C} & 1.83864 & -1.56905 & -0.57508 & \mathrm{H} & -4.18241 & -0.31393 & -0.64977 \\ \mathrm{C} & -2.64488 & -2.86021 & 0.68834 & \mathrm{H} & -3.49275 & 1.50828 & -3.94781 \\ \mathrm{C} & 4.15903 & -1.88030 & 0.28436 & \mathrm{H} & 5.66054 & 0.16309 & -2.71795\end{array}$




\begin{tabular}{|c|c|c|c|c|c|c|c|}
\hline $\mathrm{C}$ & 1.19513 & -1.94652 & 0.77817 & $\mathrm{H}$ & 1.89689 & -0.14769 & 1.80796 \\
\hline $\mathrm{C}$ & -1.91041 & 2.73940 & 1.22301 & $\mathrm{H}$ & 0.61329 & -1.00784 & 2.65727 \\
\hline $\mathrm{C}$ & -1.32341 & -2.47534 & 1.33609 & $\mathrm{H}$ & 0.23746 & -0.01542 & 1.24116 \\
\hline $\mathrm{C}$ & 2.13491 & -2.94564 & 1.46492 & $\mathrm{H}$ & -0.68963 & -2.38714 & -1.78898 \\
\hline $\mathrm{C}$ & 3.54352 & -2.35411 & 1.61404 & $\mathrm{H}$ & -2.47847 & 0.68111 & 0.81108 \\
\hline $\mathrm{C}$ & -2.55525 & -0.95953 & -1.94224 & $\mathrm{H}$ & -1.80037 & 1.67963 & 3.07726 \\
\hline $\mathrm{C}$ & 3.21524 & -0.92005 & -0.46958 & $\mathrm{H}$ & 8.24639 & -1.39129 & 1.53270 \\
\hline $\mathrm{C}$ & -6.04171 & -0.23442 & -2.36450 & $\mathrm{H}$ & 8.67643 & -2.97077 & 0.87361 \\
\hline $\mathrm{C}$ & 6.05203 & -0.58504 & -0.80519 & $\mathrm{H}$ & 4.81029 & 0.35131 & 1.62983 \\
\hline $\mathrm{C}$ & -3.78260 & -0.09984 & -1.64526 & $\mathrm{H}$ & 6.56738 & 0.26789 & 1.64611 \\
\hline $\mathrm{C}$ & -2.80100 & 1.69234 & -3.12032 & $\mathrm{H}$ & 5.63181 & -0.88469 & 2.60463 \\
\hline $\mathrm{C}$ & 5.23869 & -0.31035 & -1.82998 & $\mathrm{H}$ & 6.31582 & -2.98690 & 1.64491 \\
\hline $\mathrm{C}$ & 0.97131 & -0.71079 & 1.66455 & $\mathrm{H}$ & 6.41546 & -3.23412 & -0.09731 \\
\hline $\mathrm{C}$ & -0.51480 & -1.66346 & -0.98508 & $\mathrm{H}$ & -4.08163 & 0.72366 & 2.55409 \\
\hline $\mathrm{C}$ & 5.59538 & -1.30971 & 0.45980 & $\mathrm{H}$ & -5.47292 & 1.82086 & 2.36004 \\
\hline $\mathrm{C}$ & -2.78541 & 1.70082 & 0.51514 & $\mathrm{H}$ & -1.11527 & 0.90319 & -4.22564 \\
\hline $\mathrm{C}$ & -2.14018 & 2.66809 & 2.72087 & $\mathrm{H}$ & -0.85638 & 1.04639 & -2.46899 \\
\hline $\mathrm{C}$ & 8.04322 & -2.09202 & 0.70968 & $\mathrm{H}$ & 1.63521 & 2.60687 & 2.16835 \\
\hline $\mathrm{C}$ & 5.65890 & -0.33894 & 1.65535 & $\mathrm{H}$ & 8.29164 & -2.15518 & -1.42027 \\
\hline $\mathrm{C}$ & 6.56772 & -2.49308 & 0.69991 & $\mathrm{H}$ & 7.75541 & 0.53795 & -0.11266 \\
\hline $\mathrm{C}$ & -4.39401 & 1.72649 & 2.22171 & $\mathrm{H}$ & 7.76286 & 0.20976 & -1.84776 \\
\hline $\mathrm{C}$ & -1.58271 & 0.77937 & -3.24722 & $\mathrm{H}$ & -3.96911 & 3.78219 & 2.71632 \\
\hline $\mathrm{C}$ & 2.21820 & 2.66786 & 1.25494 & $\mathrm{H}$ & -0.26627 & -3.05101 & 3.15056 \\
\hline $\mathrm{C}$ & 8.42463 & -1.42953 & -0.60758 & $\mathrm{H}$ & -0.82036 & -4.41786 & 2.16589 \\
\hline $\mathrm{C}$ & 1.56664 & 2.84218 & 0.02645 & $\mathrm{H}$ & -1.97182 & -3.49613 & 3.15693 \\
\hline $\mathrm{C}$ & 0.09979 & 3.03160 & -0.05059 & $\mathrm{H}$ & 4.12531 & 2.52846 & 2.25056 \\
\hline $\mathrm{C}$ & 7.51951 & -0.22470 & -0.87186 & $\mathrm{H}$ & -3.91686 & -2.48143 & 2.33517 \\
\hline $\mathrm{C}$ & -3.62985 & 2.78157 & 3.01772 & $\mathrm{H}$ & -3.74210 & -1.13719 & 1.24110 \\
\hline $\mathrm{C}$ & -1.08188 & -3.41982 & 2.52503 & $\mathrm{H}$ & 1.80735 & 3.08456 & -2.09108 \\
\hline $\mathrm{C}$ & 3.59969 & 2.62230 & 1.30598 & $\mathrm{H}$ & 4.27034 & 2.92621 & -2.03132 \\
\hline $\mathrm{C}$ & -3.88949 & -2.22730 & 1.26680 & $\mathrm{H}$ & -5.36546 & -3.69369 & 0.71588 \\
\hline $\mathrm{C}$ & 2.31895 & 2.94217 & -1.14373 & $\mathrm{H}$ & -5.06431 & -2.48210 & -0.51648 \\
\hline $\mathrm{C}$ & 4.35396 & 2.71874 & 0.12507 & $\mathrm{H}$ & -6.20627 & -0.75965 & 0.84005 \\
\hline $\mathrm{C}$ & 3.70648 & 2.86525 & -1.10811 & $\mathrm{H}$ & -6.74555 & -3.04400 & 2.81346 \\
\hline $\mathrm{C}$ & -5.18365 & -2.62075 & 0.56809 & $\mathrm{H}$ & -7.54669 & -1.46437 & 2.86044 \\
\hline $\mathrm{C}$ & -6.39878 & -1.82157 & 1.05122 & $\mathrm{H}$ & -5.80790 & -1.57669 & 3.14852 \\
\hline $\mathrm{C}$ & -6.63262 & -1.98362 & 2.55447 & $\mathrm{H}$ & -8.50899 & -1.63211 & 0.55180 \\
\hline $\mathrm{C}$ & -7.64433 & -2.24264 & 0.27259 & $\mathrm{H}$ & -7.88893 & -3.29122 & 0.48208 \\
\hline $\mathrm{C}$ & 6.48848 & 2.98910 & -0.86211 & $\mathrm{H}$ & -7.49374 & -2.14040 & -0.80732 \\
\hline $\mathrm{H}$ & -0.81981 & -4.17460 & -0.42723 & $\mathrm{H}$ & 6.22475 & 3.97420 & -1.26198 \\
\hline $\mathrm{H}$ & -1.92852 & 3.22708 & -2.34667 & $\mathrm{H}$ & 6.36870 & 2.22819 & -1.63942 \\
\hline $\mathrm{H}$ & -1.72026 & 3.70847 & 4.27060 & $\mathrm{H}$ & 7.52092 & 2.99802 & -0.51469 \\
\hline
\end{tabular}


Figure S2. Calculated ECD spectra of conformers in 1-Ba-l, 1-C, and the non-disordered monomer in crystal structure
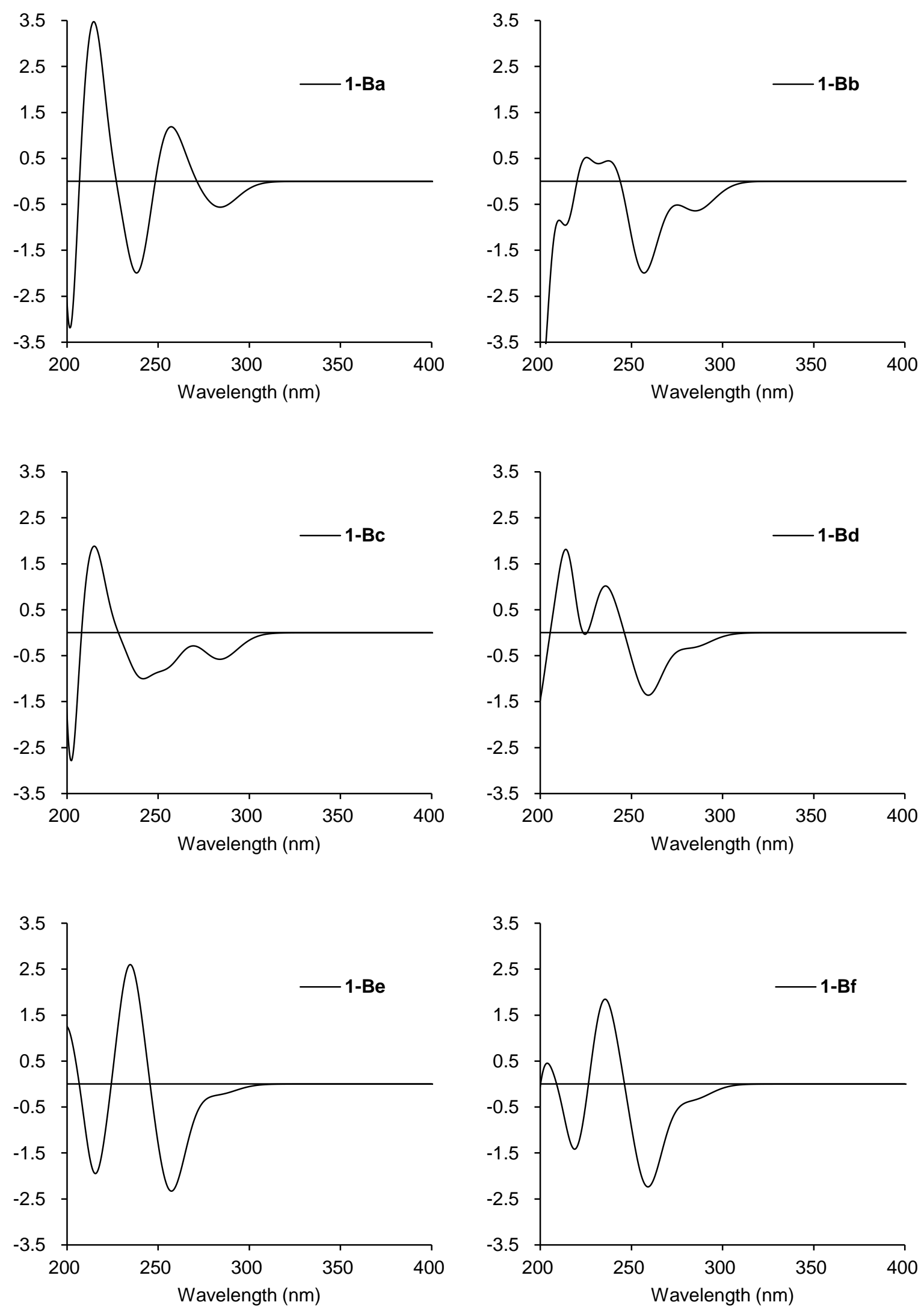

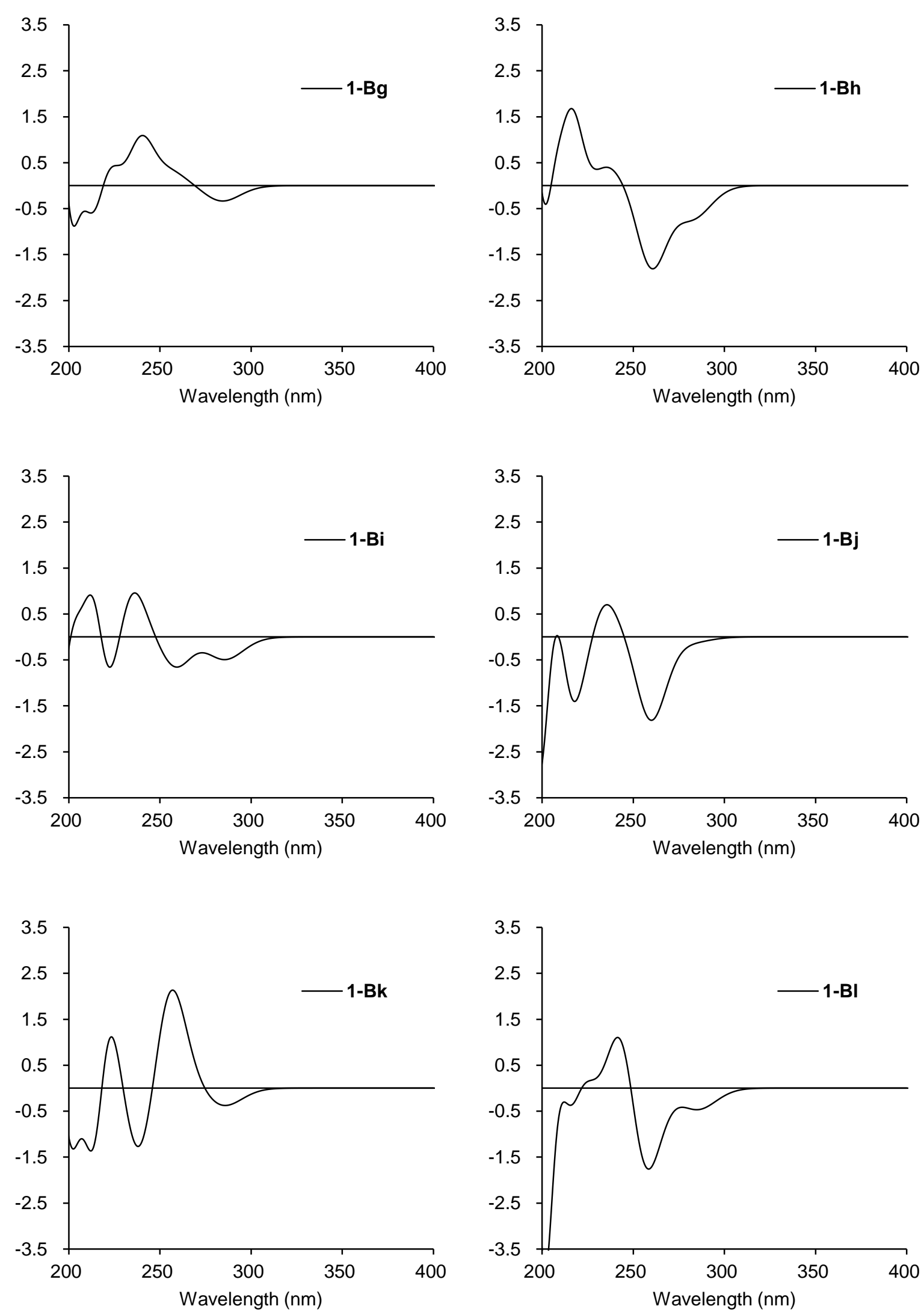

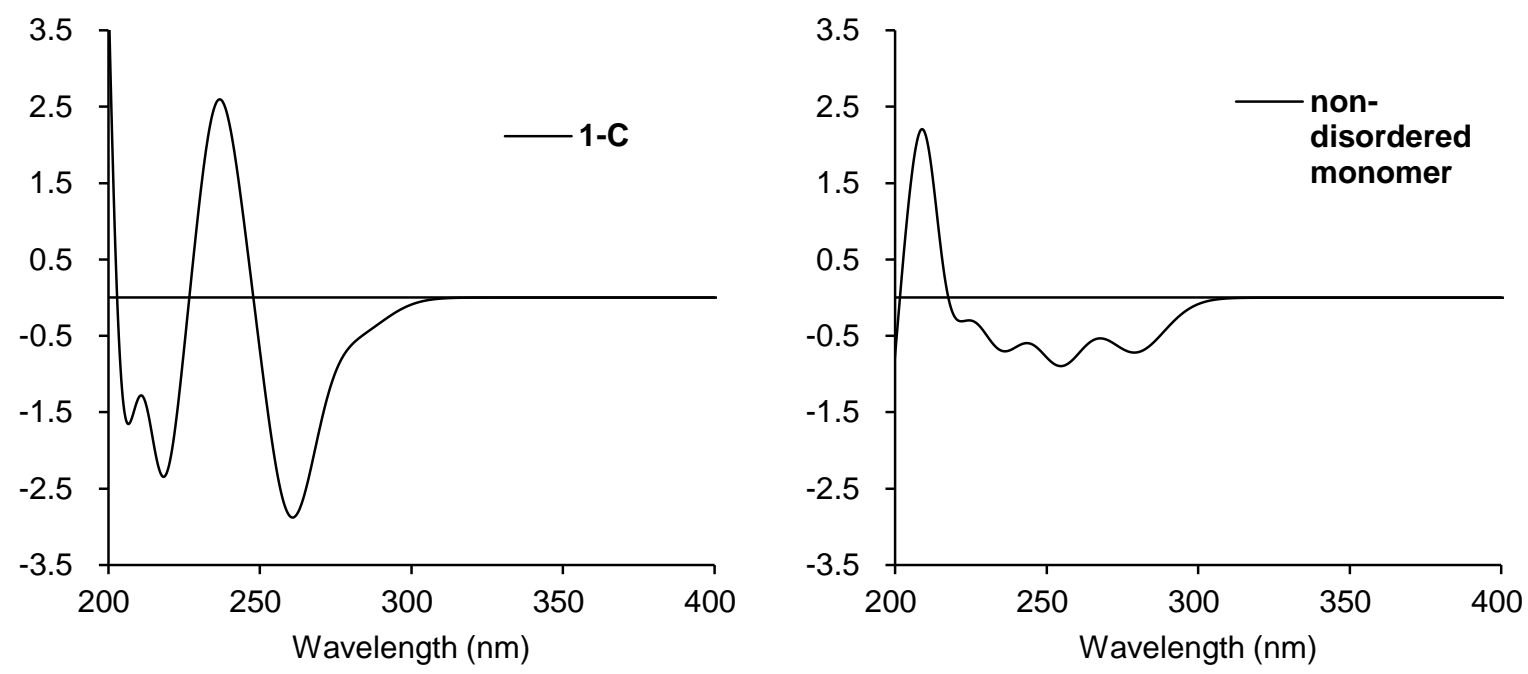
Table S3. Comparison of experimental and calculated NMR chemical shifts, the DP4+ analysis and CMAE

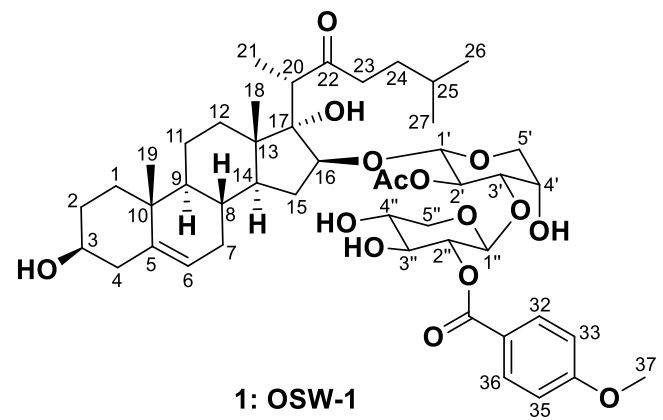

${ }^{13} \mathrm{C}$ NMR data in $\mathrm{CD}_{3} \mathrm{OD}, 125 \mathrm{MHz}$ [ppm, unscaled] ${ }^{*}$

\begin{tabular}{|c|c|c|c|c|c|}
\hline position & Experimental & $1-A$ & 1-B & $1-\mathrm{C}$ & X-ray ${ }^{* *}$ \\
\hline 1 & 38.5 & 37.6 & 37.5 & 37.8 & 23.5 \\
\hline 2 & 32.3 & 36.7 & 35.9 & 38.4 & 15.0 \\
\hline 3 & 72.3 & 73.2 & 73.1 & 73.4 & 66.3 \\
\hline 4 & 43.0 & 45.5 & 45.2 & 46.1 & 26.7 \\
\hline 5 & 142.2 & 141.7 & 141.2 & 142.5 & 145.4 \\
\hline 6 & 122.2 & 123.0 & 122.8 & 123.3 & 115.5 \\
\hline 7 & 33.5 & 34.8 & 34.7 & 34.9 & 16.8 \\
\hline 8 & 33.2 & 34.0 & 35.3 & 31.6 & 22.0 \\
\hline 9 & 51.3 & 52.1 & 52.1 & 52.0 & 38.8 \\
\hline 10 & 37.6 & 45.0 & 44.8 & 45.4 & 35.5 \\
\hline 11 & 21.6 & 24.7 & 24.8 & 24.4 & 8.0 \\
\hline 12 & 35.2 & 36.2 & 36.7 & 35.1 & 19.4 \\
\hline 13 & 47.2 & 48.8 & 48.8 & 48.9 & 49.3 \\
\hline 14 & 49.4 & 50.5 & 50.6 & 50.4 & 41.1 \\
\hline 15 & 40.1 & 38.1 & 38.0 & 38.3 & 23.3 \\
\hline 16 & 89.1 & 88.8 & 87.6 & 91.0 & 80.7 \\
\hline 17 & 86.7 & 87.4 & 86.9 & 88.4 & 86.0 \\
\hline 18 & 13.9 & 16.4 & 15.2 & 18.7 & -6.4 \\
\hline 19 & 19.8 & 22.1 & 22.0 & 22.2 & 1.5 \\
\hline 20 & 47.0 & 49.8 & 49.4 & 50.7 & 42.7 \\
\hline 21 & 12.1 & 13.5 & 12.5 & 15.3 & -5.1 \\
\hline 22 & 220.6 & 218.9 & 219.8 & 216.9 & 215.0 \\
\hline 23 & 33.9 & 43.0 & 42.1 & 44.8 & 24.0 \\
\hline 24 & 33.1 & 33.7 & 33.4 & 34.2 & 12.3 \\
\hline
\end{tabular}




\begin{tabular}{cccccc}
25 & 28.6 & 28.3 & 27.6 & 29.8 & 11.6 \\
$\mathrm{CH}_{3}$ of Ac & 20.9 & 24.6 & 25.5 & 22.7 & 7.9 \\
$\mathrm{C}=\mathrm{O}$ of Ac & 170.7 & 169.1 & 169.5 & 168.4 & 165.1 \\
1' $^{\prime}$ & 101.5 & 99.7 & 99.9 & 99.3 & 94.3 \\
$2^{\prime}$ & 72.4 & 70.5 & 71.1 & 69.3 & 70.0 \\
$3^{\prime}$ & 81.1 & 77.9 & 82.2 & 69.2 & 74.8 \\
$4^{\prime}$ & 70.9 & 68.3 & 69.5 & 65.8 & 63.1 \\
$5^{\prime}$ & 65.5 & 64.8 & 66.6 & 61.4 & 53.6 \\
1" $^{\prime \prime}$ & 103.8 & 100.5 & 102.1 & 97.3 & 99.8 \\
$2^{\prime \prime}$ & 75.8 & 76.4 & 74.1 & 81.0 & 66.8 \\
$3^{\prime \prime}$ & 75.1 & 75.8 & 76.6 & 74.2 & 70.2 \\
$4^{\prime \prime}$ & 68.8 & 71.4 & 70.4 & 73.6 & 63.8 \\
5" & 66.6 & 67.5 & 66.7 & 69.1 & 52.9 \\
PMB ipso-C=O & 123.5 & 122.1 & 121.6 & 123.3 & 123.1 \\
32, 36 & 133.2 & 131.3 & 131.0 & 131.7 & 124.8 \\
33, 35 & 114.8 & 113.0 & 112.0 & 115.0 & 106.0 \\
$\mathrm{C}=\mathrm{O}$ of PMB & 167.0 & 163.3 & 161.9 & 166.1 & 160.7 \\
PMB ipso-OMe & 165.3 & 160.5 & 160.2 & 161.1 & 159.4 \\
37 & 56.0 & 55.6 & 55.1 & 56.5 & 38.4 \\
\hline
\end{tabular}

${ }^{*} \mathrm{C} 26$ and $\mathrm{C} 27$ were not assigned. TMS was used as a reference for calculated shift. ${ }^{* *} \mathrm{X}$-ray represents the non-disordered monomer in crystal structure.

${ }^{13} \mathrm{C}$ NMR data in $\mathrm{CD}_{3} \mathrm{OD}, 125 \mathrm{MHz}\left[\mathrm{ppm}\right.$, scaled] ${ }^{*}$

\begin{tabular}{cccccc}
\hline position & Experimental & $\mathbf{1 - A}$ & $\mathbf{1 - B}$ & $\mathbf{1 - C}$ & $|\mathbf{E x p .}-\mathbf{1 - A}|$ \\
\hline 1 & 38.5 & 36.0 & 35.9 & 36.1 & 2.5 \\
2 & 32.3 & 35.1 & 34.3 & 36.7 & 2.8 \\
3 & 72.3 & 72.8 & 72.8 & 72.8 & 0.5 \\
4 & 43.0 & 44.1 & 43.9 & 44.7 & 1.2 \\
5 & 142.2 & 143.5 & 143.0 & 144.2 & 1.3 \\
6 & 122.2 & 124.2 & 124.0 & 124.4 & 2.0 \\
7 & 33.5 & 33.1 & 33.1 & 33.2 & 0.4 \\
8 & 33.2 & 32.3 & 33.7 & 29.7 & 0.8 \\
9 & 51.3 & 50.9 & 51.0 & 50.8 & 0.4 \\
10 & 37.6 & 43.7 & 43.5 & 44.0 & 6.0 \\
11 & 21.6 & 22.6 & 22.8 & 22.3 & 1.0
\end{tabular}




\begin{tabular}{|c|c|c|c|c|c|}
\hline 12 & 35.2 & 34.5 & 35.1 & 33.4 & 0.7 \\
\hline 13 & 47.2 & 47.6 & 47.6 & 47.6 & 0.3 \\
\hline 14 & 49.4 & 49.3 & 49.5 & 49.1 & 0.1 \\
\hline 15 & 40.1 & 36.6 & 36.5 & 36.7 & 3.5 \\
\hline 16 & 89.1 & 88.9 & 87.7 & 91.1 & 0.3 \\
\hline 17 & 86.7 & 87.4 & 86.9 & 88.3 & 0.7 \\
\hline 18 & 13.9 & 14.1 & 13.0 & 16.5 & 0.3 \\
\hline 19 & 19.8 & 20.0 & 20.0 & 20.1 & 0.1 \\
\hline 20 & 47.0 & 48.6 & 48.2 & 49.5 & 1.6 \\
\hline 21 & 12.1 & 11.1 & 10.2 & 13.0 & 1.0 \\
\hline 22 & 220.6 & 223.2 & 224.2 & 221.0 & 2.6 \\
\hline 23 & 33.9 & 41.6 & 40.7 & 43.4 & 7.7 \\
\hline 24 & 33.1 & 31.9 & 31.7 & 32.5 & 1.1 \\
\hline 25 & 28.6 & 26.4 & 25.7 & 27.9 & 2.2 \\
\hline $\mathrm{CH}_{3}$ of $\mathrm{Ac}$ & 20.9 & 22.5 & 23.6 & 20.5 & 1.6 \\
\hline $\mathrm{C}=\mathrm{O}$ of $\mathrm{Ac}$ & 170.7 & 171.9 & 172.2 & 170.9 & 1.2 \\
\hline $1^{\prime}$ & 101.5 & 100.1 & 100.4 & 99.6 & 1.3 \\
\hline $2^{\prime}$ & 72.4 & 70.0 & 70.7 & 68.7 & 2.3 \\
\hline $3^{\prime}$ & 81.1 & 77.6 & 82.1 & 68.5 & 3.5 \\
\hline $4^{\prime}$ & 70.9 & 67.7 & 69.0 & 65.0 & 3.2 \\
\hline $5^{\prime}$ & 65.5 & 64.1 & 66.0 & 60.5 & 1.4 \\
\hline $1 "$ & 103.8 & 101.0 & 102.6 & 97.6 & 2.8 \\
\hline $2^{\prime \prime}$ & 75.8 & 76.0 & 73.7 & 80.7 & 0.2 \\
\hline $3 "$ & 75.1 & 75.5 & 76.4 & 73.8 & 0.4 \\
\hline $4 "$ & 68.8 & 70.9 & 69.9 & 73.1 & 2.2 \\
\hline $5^{\prime \prime}$ & 66.6 & 66.9 & 66.1 & 68.4 & 0.3 \\
\hline $\mathrm{PMB}$ ipso- $\mathrm{C}=\mathrm{O}$ & 123.5 & 123.3 & 122.7 & 124.3 & 0.2 \\
\hline 32,36 & 133.2 & 132.7 & 132.5 & 133.1 & 0.5 \\
\hline 33,35 & 114.8 & 113.9 & 112.9 & 115.9 & 0.9 \\
\hline $\mathrm{C}=\mathrm{O}$ of $\mathrm{PMB}$ & 167.0 & 165.9 & 164.4 & 168.5 & 1.1 \\
\hline PMB ipso-OMe & 165.3 & 163.0 & 162.6 & 163.4 & 2.4 \\
\hline \multirow[t]{3}{*}{37} & 56.0 & 54.6 & 54.1 & 55.4 & 1.5 \\
\hline & slope & 1.033 & 1.032 & 1.032 & \\
\hline & intercept & -2.842 & -2.722 & -2.849 & \\
\hline
\end{tabular}

${ }^{*} \mathrm{C} 26$ and $\mathrm{C} 27$ were not assigned. 
${ }^{1} \mathrm{H}$ NMR data in $\mathrm{CD}_{3} \mathrm{OD}, 500 \mathrm{MHz}$ [ppm, unscaled] ${ }^{*}$

\begin{tabular}{|c|c|c|c|c|}
\hline position & Experimental & 1-A & 1-B & $1-C$ \\
\hline lax & $1.07(1 \mathrm{H}, \mathrm{m})$ & 1.12 & 1.15 & 1.08 \\
\hline leq & $1.86(1 \mathrm{H}, \mathrm{d}, 20.4)$ & 2.13 & 2.18 & 2.03 \\
\hline 3 & $3.38(1 \mathrm{H}, \mathrm{m})$ & 3.48 & 3.55 & 3.35 \\
\hline 6 & $5.33(1 \mathrm{H}, \mathrm{d}, 5.5)$ & 5.75 & 5.77 & 5.73 \\
\hline 8 & $1.52(1 \mathrm{H}, \mathrm{m})$ & 0.83 & 1.40 & -0.33 \\
\hline 9 & $0.89(1 \mathrm{H}, \mathrm{m})$ & 0.83 & 0.97 & 0.55 \\
\hline $11 \mathrm{ax}$ & $1.58(1 \mathrm{H}, \mathrm{m})$ & 1.65 & 1.70 & 1.55 \\
\hline $11 \mathrm{eq}$ & $1.48(1 \mathrm{H}, \mathrm{m})$ & 1.55 & 1.61 & 1.43 \\
\hline $12 \mathrm{ax}$ & $1.32(1 \mathrm{H}, \mathrm{m})$ & 1.72 & 1.71 & 1.74 \\
\hline $12 \mathrm{eq}$ & $2.19(1 \mathrm{H}, \mathrm{m})$ & 1.36 & 1.32 & 1.45 \\
\hline 14 & $1.65(1 \mathrm{H}, \mathrm{m})$ & 1.57 & 1.73 & 1.24 \\
\hline 15ax (trans to H14) & $2.21(1 \mathrm{H}, \mathrm{m})$ & 1.10 & 1.19 & 0.91 \\
\hline 15eq (cis to H14) & $2.48(1 \mathrm{H}, \mathrm{m})$ & 2.08 & 2.17 & 1.91 \\
\hline 16 & $3.76(1 \mathrm{H}, \mathrm{m})$ & 3.54 & 3.53 & 3.57 \\
\hline 18 & $0.81(3 \mathrm{H}, \mathrm{s})$ & 0.81 & 0.79 & 0.85 \\
\hline 19 & $1.01(3 \mathrm{H}, \mathrm{s})$ & 1.19 & 1.16 & 1.26 \\
\hline 20 & $2.91(1 \mathrm{H}, \mathrm{q}, 7.5)$ & 2.92 & 2.92 & 2.91 \\
\hline 21 & $1.10(3 \mathrm{H}, \mathrm{d}, 7.5)$ & 1.12 & 1.05 & 1.26 \\
\hline 25 & $1.26(1 \mathrm{H}, \mathrm{m})$ & 1.19 & 0.84 & 1.90 \\
\hline $\mathrm{CH}_{3}$ of $\mathrm{Ac}$ & $1.69(3 \mathrm{H}, \mathrm{s})$ & 1.89 & 1.76 & 2.15 \\
\hline 32,36 & $8.04(2 \mathrm{H}, \mathrm{d}, 9.2)$ & 8.55 & 8.58 & 8.47 \\
\hline 33,35 & $7.01(2 \mathrm{H}, \mathrm{d}, 8.6)$ & 7.16 & 7.16 & 7.16 \\
\hline 37 & $3.87(3 \mathrm{H}, \mathrm{s})$ & 4.02 & 4.03 & 4.00 \\
\hline $1^{\prime}$ & $4.14(1 \mathrm{H}, \mathrm{d}, 6.4)$ & 3.95 & 3.86 & 4.14 \\
\hline $2^{\prime}$ & $4.86(1 \mathrm{H}, \mathrm{dd}, 8.5,6.4)$ & 5.03 & 5.24 & 4.61 \\
\hline $3^{\prime}$ & $3.76(1 \mathrm{H}, \mathrm{dd}, 8.5,3.5)$ & 3.86 & 3.45 & 4.68 \\
\hline $4^{\prime}$ & $3.94(1 \mathrm{H}, \mathrm{ddd}, 4.1,3.5,2.3)$ & 3.92 & 3.92 & 3.92 \\
\hline $5^{\prime} \mathrm{a}$ (cis to H4') & $3.47(1 \mathrm{H}, \mathrm{dd}, 12.4,2.3)$ & 3.50 & 3.51 & 3.49 \\
\hline 5'b (trans to H4') & $3.82(1 \mathrm{H}, \mathrm{dd}, 12.4,4.6)$ & 3.96 & 4.07 & 3.75 \\
\hline $1 "$ & $4.66(1 \mathrm{H}, \mathrm{d}, 7.3)$ & 4.71 & 4.49 & 5.16 \\
\hline $2^{\prime \prime}$ & $4.94(1 \mathrm{H}, \mathrm{dd}, 8.5,7.7)$ & 4.87 & 4.95 & 4.72 \\
\hline $3 "$ & $3.54(1 \mathrm{H}, \mathrm{dd}, 8.5,8.3)$ & 3.89 & 3.70 & 4.26 \\
\hline $4 "$ & $3.60(1 \mathrm{H}, \mathrm{dd}, 8.2,8.0,4.0)$ & 3.72 & 3.81 & 3.55 \\
\hline
\end{tabular}


5"ax (trans to H4") $\quad 3.31(1 \mathrm{H}, \mathrm{dd}, 11.6,9.3) \quad 3.60 \quad 3.46 \quad 3.88$

5"eq (cis to H4") $\quad 3.96(1 \mathrm{H}, \mathrm{dd}, 11.7,5.0) \quad 4.12 \quad 4.05 \quad 4.25$

*H2ax, H2eq, H3ax, H4eq, H7ax, H7eq, H23a, H23b, H24a, H24b, H26 and H27 were not assigned. TMS was used as a reference for calculated shift.

${ }^{1} \mathrm{H}$ NMR data in $\mathrm{CD}_{3} \mathrm{OD}, 500 \mathrm{MHz}$ [ppm, scaled] ${ }^{*}$

\begin{tabular}{|c|c|c|c|c|c|}
\hline position & Experimental & 1-A & 1-B & $1-C$ & $\begin{array}{c}\mid \text { Exp. - } \\
1-\mathbf{A} \mid \\
\end{array}$ \\
\hline $1 \mathrm{ax}$ & $1.07(1 \mathrm{H}, \mathrm{m})$ & 1.27 & 1.28 & 1.34 & 0.20 \\
\hline 1 eq & $1.86(1 \mathrm{H}, \mathrm{d}, 20.4)$ & 2.20 & 2.23 & 2.16 & 0.34 \\
\hline 3 & $3.38(1 \mathrm{H}, \mathrm{m})$ & 3.44 & 3.50 & 3.30 & 0.06 \\
\hline 6 & $5.33(1 \mathrm{H}, \mathrm{d}, 5.5)$ & 5.52 & 5.55 & 5.36 & 0.19 \\
\hline 8 & $1.52(1 \mathrm{H}, \mathrm{m})$ & 1.00 & 1.52 & 0.12 & 0.52 \\
\hline 9 & $0.89(1 \mathrm{H}, \mathrm{m})$ & 1.01 & 1.12 & 0.89 & 0.12 \\
\hline $11 \mathrm{ax}$ & $1.58(1 \mathrm{H}, \mathrm{m})$ & 1.76 & 1.80 & 1.75 & 0.18 \\
\hline $11 \mathrm{eq}$ & $1.48(1 \mathrm{H}, \mathrm{m})$ & 1.67 & 1.71 & 1.65 & 0.19 \\
\hline $12 \mathrm{ax}$ & $1.32(1 \mathrm{H}, \mathrm{m})$ & 1.82 & 1.80 & 1.91 & 0.50 \\
\hline $12 \mathrm{eq}$ & $2.19(1 \mathrm{H}, \mathrm{m})$ & 1.50 & 1.44 & 1.66 & 0.69 \\
\hline 14 & $1.65(1 \mathrm{H}, \mathrm{m})$ & 1.68 & 1.82 & 1.48 & 0.03 \\
\hline $15 \mathrm{ax}$ (trans to $\mathrm{H} 14$ ) & $2.21(1 \mathrm{H}, \mathrm{m})$ & 1.25 & 1.32 & 1.20 & 0.96 \\
\hline 15eq (cis to H14) & $2.48(1 \mathrm{H}, \mathrm{m})$ & 2.15 & 2.22 & 2.06 & 0.33 \\
\hline 16 & $3.76(1 \mathrm{H}, \mathrm{m})$ & 3.49 & 3.48 & 3.49 & 0.27 \\
\hline 18 & $0.81(3 \mathrm{H}, \mathrm{s})$ & 0.98 & 0.95 & 1.14 & 0.17 \\
\hline 19 & $1.01(3 \mathrm{H}, \mathrm{s})$ & 1.34 & 1.29 & 1.50 & 0.33 \\
\hline 20 & $2.91(1 \mathrm{H}, \mathrm{q}, 7.5)$ & 2.92 & 2.92 & 2.93 & 0.01 \\
\hline 21 & $1.10(3 \mathrm{H}, \mathrm{d}, 7.5)$ & 1.27 & 1.19 & 1.50 & 0.17 \\
\hline 25 & $1.26(1 \mathrm{H}, \mathrm{m})$ & 1.34 & 1.00 & 2.05 & 0.08 \\
\hline $\mathrm{CH}_{3}$ of $\mathrm{Ac}$ & $1.69(3 \mathrm{H}, \mathrm{s})$ & 1.98 & 1.85 & 2.27 & 0.29 \\
\hline 32,36 & $8.04(2 \mathrm{H}, \mathrm{d}, 9.2)$ & 8.08 & 8.15 & 7.73 & 0.04 \\
\hline 33,35 & $7.01(2 \mathrm{H}, \mathrm{d}, 8.6)$ & 6.81 & 6.84 & 6.60 & 0.20 \\
\hline 37 & $3.87(3 \mathrm{H}, \mathrm{s})$ & 3.93 & 3.94 & 3.86 & 0.06 \\
\hline $1^{\prime}$ & $4.14(1 \mathrm{H}, \mathrm{d}, 6.4)$ & 3.87 & 3.79 & 3.99 & 0.27 \\
\hline $2^{\prime}$ & $4.86(1 \mathrm{H}, \mathrm{dd}, 8.5,6.4)$ & 4.86 & 5.06 & 4.39 & 0.00 \\
\hline $3^{\prime}$ & $3.76(1 \mathrm{H}, \mathrm{dd}, 8.5,3.5)$ & 3.78 & 3.41 & 4.45 & 0.02 \\
\hline $4^{\prime}$ & $3.94(1 \mathrm{H}, \mathrm{ddd}, 4.1,3.5,2.3)$ & 3.84 & 3.84 & 3.80 & 0.10 \\
\hline 5'a (cis to H4') & $3.47(1 \mathrm{H}, \mathrm{dd}, 12.4,2.3)$ & 3.46 & 3.47 & 3.43 & 0.01 \\
\hline
\end{tabular}




\begin{tabular}{cccccc} 
5'b (trans to H4') & $3.82(1 \mathrm{H}, \mathrm{dd}, 12.4,4.6)$ & 3.88 & 3.98 & 3.65 & 0.06 \\
1" & $4.66(1 \mathrm{H}, \mathrm{d}, 7.3)$ & 4.57 & 4.37 & 4.87 & 0.09 \\
2" & $4.94(1 \mathrm{H}, \mathrm{dd}, 8.5,7.7)$ & 4.72 & 4.80 & 4.49 & 0.22 \\
3" & $3.54(1 \mathrm{H}, \mathrm{dd}, 8.5,8.3)$ & 3.81 & 3.64 & 4.09 & 0.27 \\
4" & $3.60(1 \mathrm{H}, \mathrm{dd}, 8.2,8.0,4.0)$ & 3.66 & 3.74 & 3.48 & 0.06 \\
5"ax (trans to H4") & $3.31(1 \mathrm{H}, \mathrm{dd}, 11.6,9.3)$ & 3.55 & 3.42 & 3.76 & 0.24 \\
5"eq (cis to H4") & $3.96(1 \mathrm{H}, \mathrm{dd}, 11.7,5.0)$ & 4.02 & 3.97 & 4.08 & 0.06 \\
& slope & 0.9171 & 0.9242 & 0.8649 & \\
& intercept & 0.2448 & 0.2219 & 0.4067 & \\
\hline
\end{tabular}

*H2ax, H2eq, H3ax, H4eq, H7ax, H7eq, H23a, H23b, H24a, H24b, H26 and H27 were not assigned.

\begin{tabular}{cccc}
\multicolumn{4}{c}{ DP4+ analysis } \\
\hline & $\mathbf{1 - A}$ & $\mathbf{1 - B}$ & $\mathbf{1 - C}$ \\
\hline DP4+ (H data) & $99.98 \%$ & $0.02 \%$ & $0.00 \%$ \\
DP4+ (C data) & $29.64 \%$ & $70.36 \%$ & $0.00 \%$ \\
DP4+ (all data) & $99.96 \%$ & $0.04 \%$ & $0.00 \%$ \\
\hline
\end{tabular}

\begin{tabular}{ccccc}
\multicolumn{5}{c}{ CMAE analysis of ${ }^{13} \mathrm{C}$ shifts } \\
\hline 1-A & 1-B & 1-C & X-ray $^{*}$ \\
\hline slope & 1.033 & 1.032 & 1.032 & 0.923 \\
intercept & -2.842 & -2.722 & -2.849 & 14.466 \\
CMAE & 1.58 & 1.59 & 2.45 & 3.82 \\
\hline
\end{tabular}

${ }^{*} \mathrm{X}$-ray represents the non-disordered monomer in crystal structure. 
Table S4. Calculated NMR chemical shifts of each conformer in 1-B

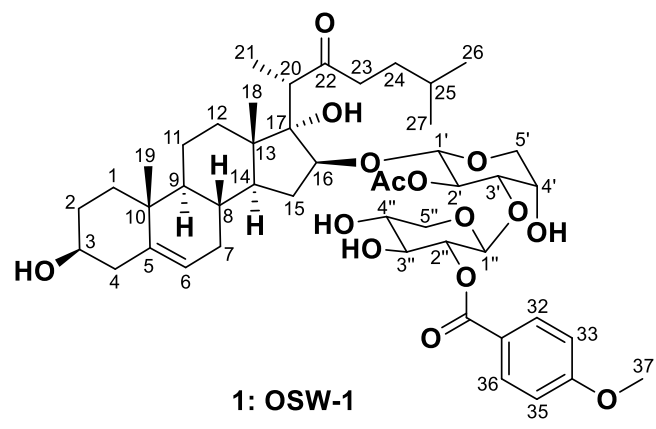

${ }^{13} \mathrm{C}$ NMR shifts $[\mathrm{ppm}] *$

\section{1-B}

position

$\begin{array}{lllllll}\mathbf{a} & \mathbf{b} & \mathbf{c} & \mathbf{d} & \mathbf{e} & \mathbf{f}\end{array}$

$\begin{array}{llllllllllll}36.1 & 40.2 & 34.6 & 40.9 & 40.8 & 40.9 & 37.7 & 36.9 & 40.3 & 37.5 & 40.8 & 41.2\end{array}$

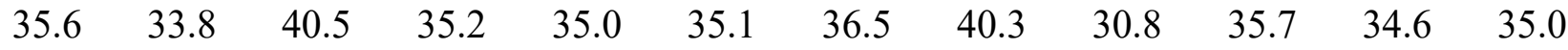

$\begin{array}{llllllllllll}73.0 & 73.9 & 73.1 & 72.3 & 73.4 & 72.2 & 73.3 & 73.5 & 72.5 & 72.4 & 73.2 & 73.3\end{array}$

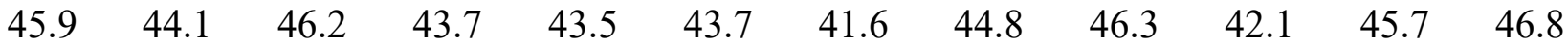

$\begin{array}{llllllllllll}141.7 & 141.7 & 140.1 & 140.6 & 139.1 & 140.9 & 142.3 & 141.3 & 141.6 & 141.0 & 142.6 & 139.6\end{array}$

$\begin{array}{lllllllllllll}6 & 122.4 & 122.4 & 123.7 & 123.7 & 123.7 & 123.7 & 122.7 & 123.0 & 121.9 & 122.0 & 121.7 & 122.4\end{array}$

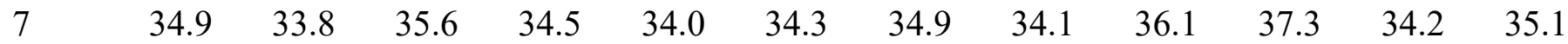

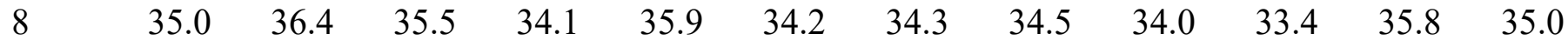

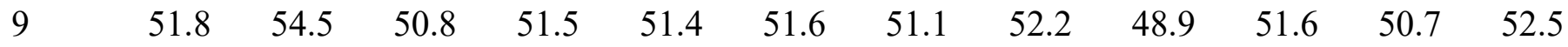

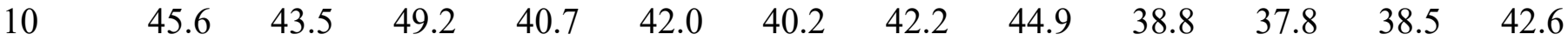

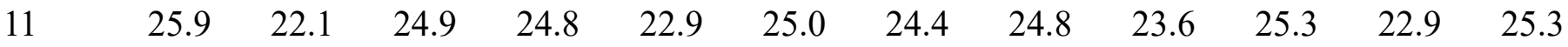

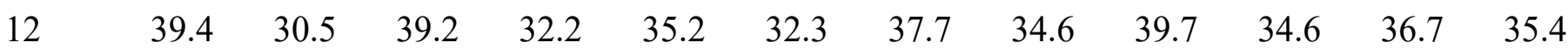

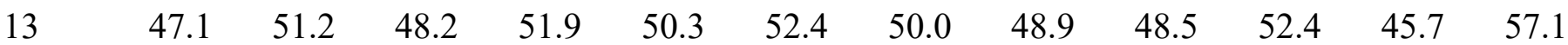

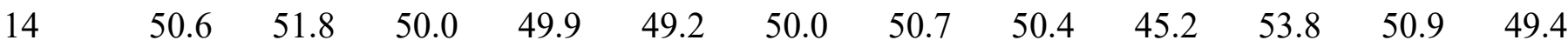

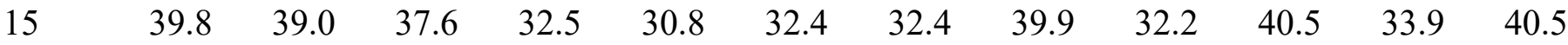

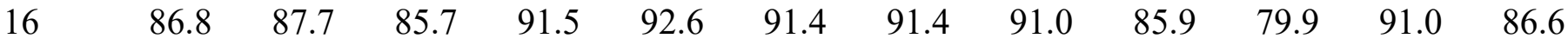

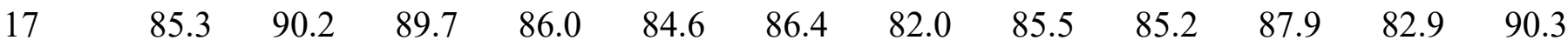

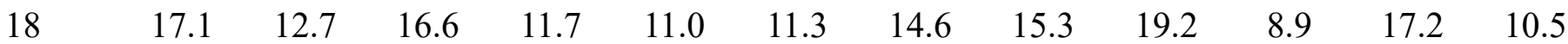

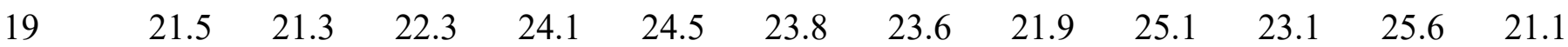

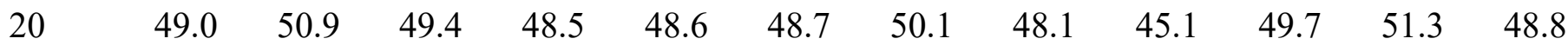

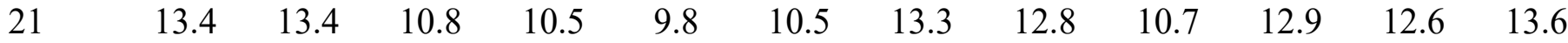

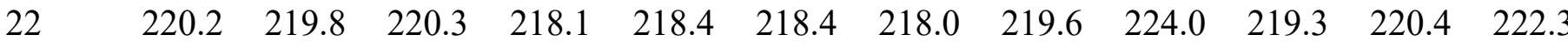

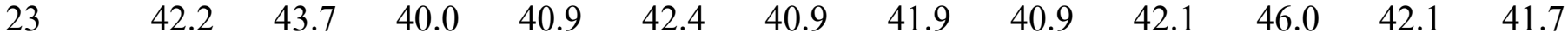

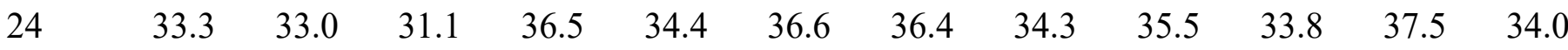

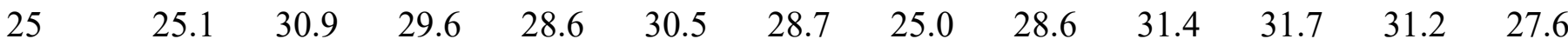




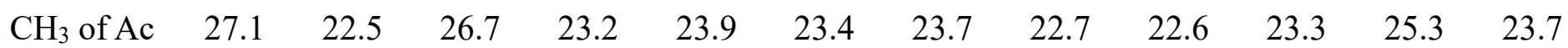

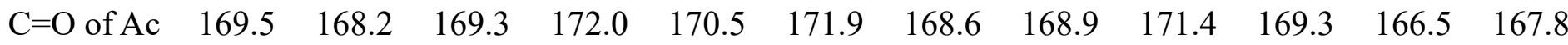

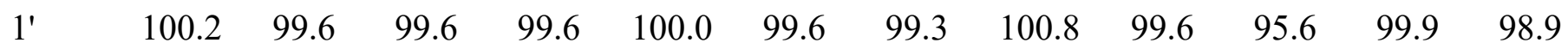

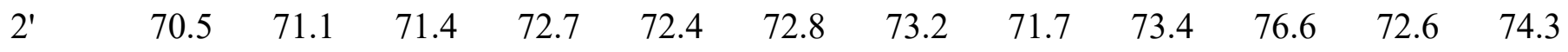

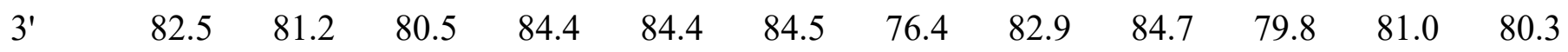

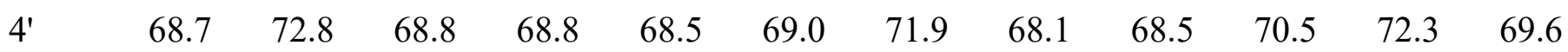
$\begin{array}{lllllllllllll}5^{\prime} & 66.9 & 65.3 & 67.1 & 66.5 & 66.8 & 66.4 & 65.7 & 66.9 & 66.1 & 68.1 & 66.3 & 66.6\end{array}$

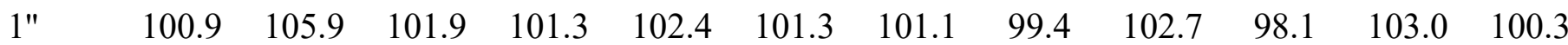

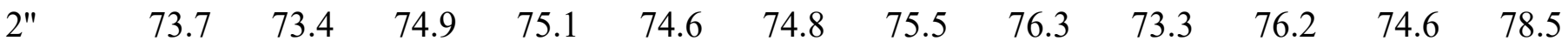

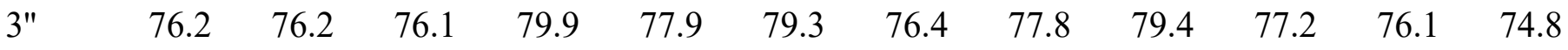

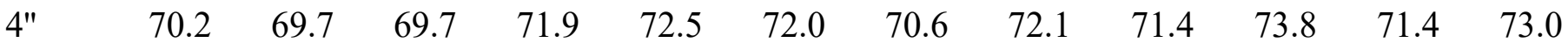

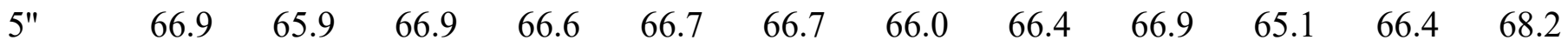

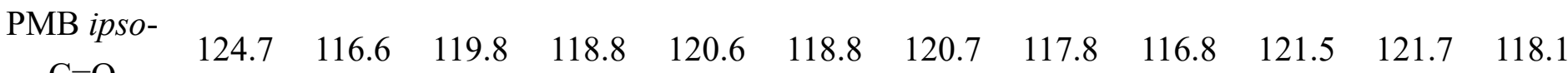
$\mathrm{C}=\mathrm{O}$

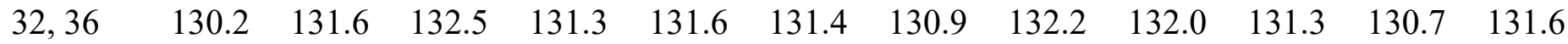

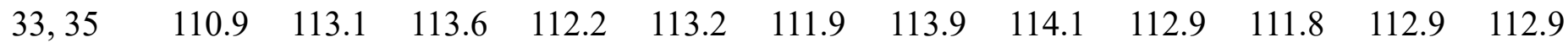

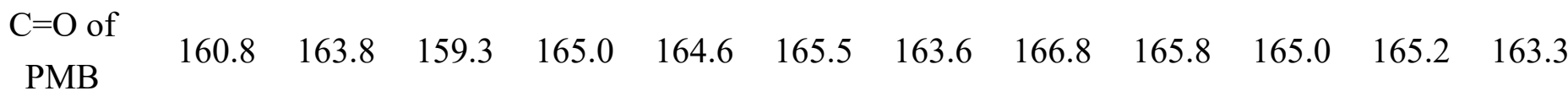

PMB ipso$\mathrm{OMe}$

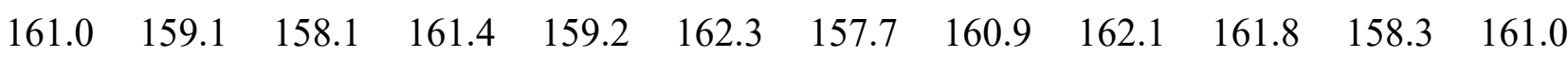

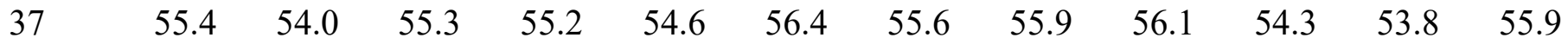

${ }^{*} \mathrm{C} 26$ and $\mathrm{C} 27$ were omitted. TMS was used as a reference.

${ }^{1} \mathrm{H}$ NMR shifts $[\mathrm{ppm}]^{*}$

\begin{tabular}{ccccccccccccc}
\hline \multirow{2}{*}{ position } & \multicolumn{1}{c}{$\mathbf{a}$} & $\mathbf{b}$ & $\mathbf{c}$ & $\mathbf{d}$ & $\mathbf{e}$ & $\mathbf{f}$ & $\mathbf{g}$ & $\mathbf{h}$ & $\mathbf{i}$ & $\mathbf{j}$ & $\mathbf{k}$ & $\mathbf{1}$ \\
\hline $1 \mathrm{ax}$ & 1.17 & 1.17 & 1.22 & 1.03 & 0.94 & 1.03 & 1.15 & 1.26 & 0.82 & 1.04 & 1.06 & 1.02 \\
$1 \mathrm{eq}$ & 2.16 & 2.27 & 2.40 & 2.07 & 1.80 & 2.05 & 1.89 & 2.19 & 1.78 & 1.90 & 1.78 & 1.91 \\
3 & 3.54 & 3.66 & 3.64 & 3.35 & 3.41 & 3.36 & 3.47 & 3.39 & 3.43 & 3.52 & 3.11 & 3.25 \\
6 & 5.83 & 5.58 & 5.78 & 5.82 & 5.76 & 5.80 & 5.79 & 5.68 & 5.73 & 5.80 & 5.73 & 5.84 \\
8 & 1.51 & 1.10 & 1.43 & 1.31 & 1.54 & 1.30 & 1.54 & 1.23 & 1.67 & 1.67 & 1.57 & 1.52 \\
9 & 1.10 & 0.79 & 0.86 & 0.83 & 0.87 & 0.83 & 1.04 & 0.97 & 1.09 & 1.06 & 1.16 & 0.91 \\
$11 \mathrm{ax}$ & 1.66 & 1.76 & 1.63 & 1.86 & 1.71 & 1.89 & 1.73 & 1.63 & 1.78 & 2.17 & 1.70 & 2.05 \\
$11 \mathrm{eq}$ & 1.68 & 1.56 & 1.57 & 1.55 & 1.45 & 1.54 & 1.50 & 1.60 & 1.26 & 1.51 & 1.69 & 1.22 \\
$12 \mathrm{ax}$ & 1.52 & 2.27 & 1.41 & 1.91 & 1.78 & 1.92 & 1.97 & 1.72 & 1.76 & 1.87 & 1.66 & 1.74 \\
$12 \mathrm{eq}$ & 1.11 & 2.31 & 0.74 & 1.34 & 1.18 & 1.36 & 1.42 & 1.52 & 0.96 & 1.68 & 1.40 & 1.56
\end{tabular}




\begin{tabular}{|c|c|c|c|c|c|c|c|c|c|c|c|c|}
\hline 14 & 1.70 & 1.92 & 1.66 & 1.64 & 1.68 & 1.62 & 2.06 & 1.50 & 1.83 & 1.91 & 1.97 & 1.97 \\
\hline $\begin{array}{c}15 \mathrm{ax} \\
\text { (trans to H14) }\end{array}$ & 1.20 & 0.95 & 1.29 & 1.30 & 1.42 & 1.33 & 1.64 & 1.18 & 1.18 & 1.08 & 1.44 & 0.85 \\
\hline $\begin{array}{c}15 \mathrm{eq} \\
\text { (cis to H14) }\end{array}$ & 2.23 & 2.12 & 2.25 & 1.94 & 1.98 & 1.95 & 2.22 & 2.14 & 1.95 & 1.95 & 2.36 & 2.01 \\
\hline 16 & 3.46 & 3.39 & 3.49 & 3.89 & 3.84 & 3.90 & 3.79 & 3.57 & 3.98 & 3.98 & 3.97 & 3.52 \\
\hline 18 & 0.82 & 0.41 & 0.89 & 1.06 & 0.96 & 1.06 & 0.95 & 0.77 & 0.85 & 0.79 & 0.87 & 0.75 \\
\hline 19 & 1.18 & 1.12 & 1.17 & 1.09 & 1.13 & 1.10 & 1.14 & 1.17 & 1.03 & 1.13 & 0.98 & 1.26 \\
\hline 20 & 3.02 & 2.58 & 3.02 & 2.94 & 2.89 & 2.94 & 2.82 & 2.74 & 3.13 & 2.82 & 3.08 & 2.67 \\
\hline 21 & 1.00 & 1.05 & 1.05 & 1.20 & 1.23 & 1.21 & 1.08 & 1.11 & 1.15 & 0.88 & 1.18 & 1.01 \\
\hline 25 & 0.32 & 1.35 & 1.41 & 1.15 & 1.50 & 1.15 & 0.12 & 1.47 & 1.98 & 1.39 & 1.12 & 2.20 \\
\hline $\mathrm{CH}_{3}$ of $\mathrm{Ac}$ & 2.30 & 0.49 & 2.48 & 1.11 & 0.82 & 1.08 & 2.32 & 0.64 & 1.01 & 0.94 & 2.48 & 0.75 \\
\hline 32,36 & 8.69 & 8.46 & 8.57 & 8.46 & 8.43 & 8.43 & 8.48 & 8.54 & 8.52 & 8.53 & 8.44 & 8.22 \\
\hline 33,35 & 7.12 & 7.14 & 7.16 & 7.28 & 7.30 & 7.29 & 7.08 & 7.37 & 7.37 & 7.36 & 7.27 & 7.31 \\
\hline 37 & 4.08 & 3.96 & 4.01 & 3.94 & 4.00 & 3.89 & 4.12 & 4.04 & 4.04 & 4.00 & 4.01 & 3.96 \\
\hline $1^{\prime}$ & 3.80 & 3.64 & 3.85 & 4.21 & 4.19 & 4.22 & 4.28 & 4.10 & 4.29 & 5.28 & 4.39 & 3.62 \\
\hline $2^{\prime}$ & 5.39 & 5.10 & 5.50 & 4.82 & 4.64 & 4.83 & 4.98 & 5.12 & 4.80 & 4.16 & 5.20 & 4.99 \\
\hline $3^{\prime}$ & 3.36 & 3.54 & 3.48 & 3.50 & 3.51 & 3.52 & 3.94 & 3.61 & 3.50 & 4.86 & 3.73 & 3.59 \\
\hline $4^{\prime}$ & 3.89 & 4.02 & 3.89 & 3.92 & 3.95 & 3.91 & 3.76 & 3.90 & 3.92 & 3.81 & 3.81 & 3.82 \\
\hline $\begin{array}{c}5^{\prime} \mathrm{a} \\
\left.\text { (cis to } \mathrm{H} 4^{\prime}\right)\end{array}$ & 3.55 & 3.35 & 3.59 & 3.49 & 3.53 & 3.50 & 3.55 & 3.48 & 3.62 & 3.46 & 3.59 & 3.39 \\
\hline $\begin{array}{c}5 ' b \\
\text { (trans to H4') }\end{array}$ & 4.05 & 4.09 & 4.11 & 4.05 & 4.10 & 4.06 & 4.05 & 4.06 & 4.10 & 3.95 & 4.06 & 4.04 \\
\hline $1 "$ & 4.43 & 4.40 & 4.56 & 4.61 & 4.74 & 4.63 & 4.66 & 4.71 & 4.69 & 4.78 & 4.54 & 5.76 \\
\hline $2^{\prime \prime}$ & 5.00 & 4.97 & 4.87 & 4.93 & 5.03 & 4.96 & 4.51 & 4.35 & 4.86 & 4.63 & 4.55 & 4.02 \\
\hline 3" & 3.75 & 3.69 & 3.87 & 3.34 & 3.55 & 3.35 & 3.80 & 3.40 & 3.43 & 3.36 & 3.92 & 4.31 \\
\hline 4" & 3.93 & 3.53 & 3.89 & 3.67 & 3.70 & 3.70 & 3.76 & 3.66 & 3.61 & 3.80 & 3.75 & 3.72 \\
\hline $\begin{array}{c}5 " \mathrm{ax} \\
\text { (trans to H4") }\end{array}$ & 3.50 & 3.49 & 3.52 & 3.21 & 3.34 & 3.24 & 3.38 & 3.46 & 3.27 & 3.48 & 3.48 & 3.72 \\
\hline $\begin{array}{c}5 \text { "eq } \\
\text { (cis to H4") }\end{array}$ & 4.06 & 4.14 & 4.01 & 3.91 & 4.02 & 3.94 & 4.00 & 4.11 & 4.00 & 4.31 & 4.14 & 4.26 \\
\hline
\end{tabular}

*H2ax, H2eq, H3ax, H4eq, H7ax, H7eq, H23a, H23b, H24a, H24b, H26 and H27 were omitted. TMS was used as a reference. 